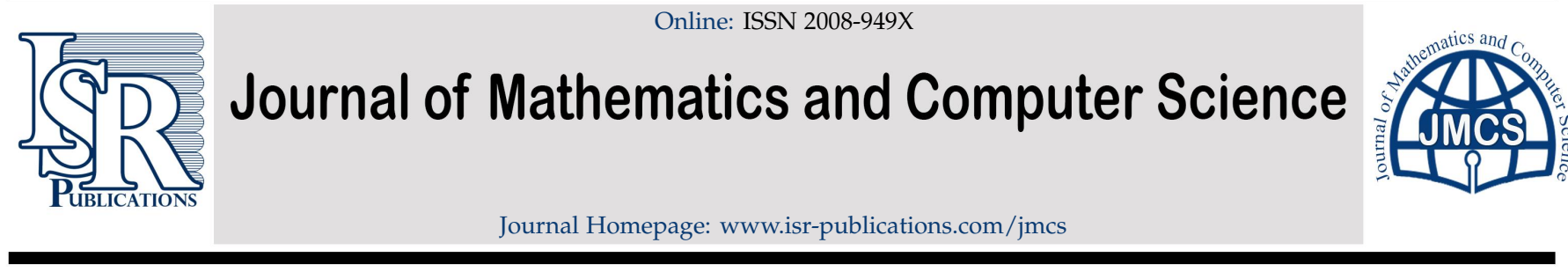

\title{
Picture fuzzy sets in UP-algebras by means of a special type
}

\author{
Sunisa Yuphaphin ${ }^{\mathrm{a}}$, Pimwaree Kankaew ${ }^{\mathrm{a}}$, Nattacha Lapo ${ }^{\mathrm{a}}$, Ronnason Chinram ${ }^{\mathrm{b}}$, Aiyared lampan ${ }^{\mathrm{a}, *}$ \\ ${ }^{a}$ Department of Mathematics, School of Science, University of Phayao, Mae Ka, Phayao 56000, Thailand. \\ ${ }^{b}$ Algebra and Applications Research Unit, Division of Computational Science, Faculty of Science, Prince of \\ Songkla University, Hat Yai, Songkhla 90110, Thailand.
}

\begin{abstract}
The concept of picture fuzzy sets was first introduced by Cuong and Kreinovich [B. C. Cuong, V. Kreinovich, Proceedings of the Third World Congress on Information and Communication Technologies WIICT, (2013), 1-6] in 2013, which is direct extensions of the fuzzy sets and the intuitionistic fuzzy sets. In this paper, we applied the concept of picture fuzzy sets in UP-algebras to introduce the eight new concepts of picture fuzzy sets by means of a special type: special picture fuzzy UPsubalgebras, special picture fuzzy near UP-filters, special picture fuzzy UP-filters, special picture fuzzy implicative UP-filters, special picture fuzzy comparative UP-filters, special picture fuzzy shift UP-filters, special picture fuzzy UP-ideals, and special picture fuzzy strong UP-ideals. Also, we discuss the relationship between the eight new concepts of picture fuzzy sets in UPalgebras. This idea is extended to the lower and upper level subsets of picture fuzzy sets in UP-algebras. Moreover, we define a picture fuzzy set in the same way as a characteristic function and study its characterizations from the related subset.
\end{abstract}

Keywords: UP-algebra, picture fuzzy set, special picture fuzzy UP-subalgebra, special picture fuzzy near UP-filter, special picture fuzzy UP-filter, special picture fuzzy implicative UP-filter, special picture fuzzy comparative UP-filter, special picture fuzzy shift UP-filter, special picture fuzzy UP-ideal, special picture fuzzy strong UP-ideal.

2020 MSC: 06F35, 08A72, $03 E 72$.

(C)2022 All rights reserved.

\section{Introduction}

Among many algebraic structures, algebras of logic form important class of algebras. Examples of these are BCK-algebras [15], BCI-algebras [16], BE-algebras [17], UP-algebras [10], fully UP-semigroups [11], topological UP-algebras [20], UP-hyperalgebras [13], extension of KU/UP-algebras [19] and others. They are strongly connected with logic. For example, BCI-algebras introduced by Iséki [16] in 1966 have connections with BCI-logic being the BCI-system in combinatory logic which has application in the language of functional programming. BCK and BCI-algebras are two classes of logical algebras. They were introduced by Imai and Iséki [15, 16] in 1966 and have been extensively investigated by many researchers.

\footnotetext{
${ }^{*}$ Corresponding author

Email addresses: sunisayuphaphin@gmail.com (Sunisa Yuphaphin), pimwaree.kk@gmail.com (Pimwaree Kankaew), nattachalapo.pp@gmail.com (Nattacha Lapo), ronnason.c@psu.ac.th (Ronnason Chinram), aiyared.ia@up.ac.th (Aiyared Iampan)
}

doi: $10.22436 /$ jmcs.025.01.05

Received: 2021-03-14 Revised: 2021-04-02 Accepted: 2021-04-07 
Fuzzy set theory, introduced by Zadeh [30] in 1965, has been widely used to model uncertainty present in real-world applications. After the introduction of the concept of fuzzy sets by Zadeh [30], Atanassov $[3,4]$ defined a new concept called an intuitionistic fuzzy set which is a generalization of fuzzy set. The concept of picture fuzzy sets was first considered by Cuong and Kreinovich [6] in 2013, which is direct extensions of the fuzzy sets and the intuitionistic fuzzy sets. The picture fuzzy set is characterized by three functions expressing the degree of membership, the degree of neutral membership, and the degree of non-membership. The only constraint is that the sum of the three degrees must not exceed 1. Cuong [5] published the concept of picture fuzzy sets in the Journal of Computer Science and Cybernetics in 2014. Some operations on picture fuzzy sets with some properties are considered. The Zadeh Extension Principle, picture fuzzy relations, and picture fuzzy soft sets are studied. Several researches were conducted on the generalizations of the concept of picture fuzzy sets in a variety of different fields and its application to a decision-making problem. In 2015, Singh [25] presented a geometrical interpretation of picture fuzzy sets. The author proposed correlation coefficients for picture fuzzy sets which considers the degree of positive membership, degree of neutral membership, degree of negative membership and the degree of refusal membership. In 2017, Wei [27] presented another form of eight similarity measures between picture fuzzy sets based on the cosine function between picture fuzzy sets by considering the degree of positive membership, degree of neutral membership, degree of negative membership and degree of refusal membership in picture fuzzy sets. The author applied these weighted cosine function similarity measures between picture fuzzy sets to strategic decision making. In 2018, Wei and Gao [29] presented some novel Dice similarity measures of picture fuzzy sets and the generalized Dice similarity measures of picture fuzzy sets and indicate that the Dice similarity measures and asymmetric measures (projection measures) are the special cases of the generalized Dice similarity measures in some parameter values. Wei [28] presented some novel process to measure the similarity between picture fuzzy sets. The author applied these similarity measures between picture fuzzy sets to building material recognition and minerals field recognition. In 2020, Ganie et al. [8] introduced two correlation coefficients of picture fuzzy sets. These correlation coefficients of picture fuzzy sets are better than existing ones and effective in expressing the nature of correlation (positive or negative correlation). As mentioned above, we find the study of picture fuzzy sets to be very important and interesting which leads us to the study of this paper.

In this paper, we applied the concept of picture fuzzy sets in UP-algebras to introduce the eight new concepts of picture fuzzy sets by means of a special type: special picture fuzzy UP-subalgebras, special picture fuzzy near UP-filters, special picture fuzzy UP-filters, special picture fuzzy implicative UP-filters, special picture fuzzy comparative UP-filters, special picture fuzzy shift UP-filters, special picture fuzzy UP-ideals, and special picture fuzzy strong UP-ideals. Also, we discuss the relationship between the eight new concepts of picture fuzzy sets in UP-algebras. This idea is extended to the lower and upper level subsets of picture fuzzy sets in UP-algebras. Moreover, we define a picture fuzzy set in the same way as a characteristic function and study its characterizations from the related subset.

\section{Basic results on UP-algebras}

Before we begin our study, let's review the definition of UP-algebras.

Definition 2.1 ([10]). An algebra $X=(X, \cdot, 0)$ of type $(2,0)$ is called a UP-algebra, where $X$ is a nonempty set, - is a binary operation on $X$, and 0 is a fixed element of $X$ (i.e., a nullary operation) if it satisfies the following axioms:

$$
\begin{aligned}
& (\forall x, y, z \in X)((y \cdot z) \cdot((x \cdot y) \cdot(x \cdot z))=0), \\
& (\forall x \in X)(0 \cdot x=x), \\
& (\forall x \in X)(x \cdot 0=0), \text { and } \\
& (\forall x, y \in X)(x \cdot y=0, y \cdot x=0 \Rightarrow x=y) .
\end{aligned}
$$


From [10], we know that the concept of UP-algebras is a generalization of KU-algebras (see [18]).

The binary relation $\leqslant$ on a UP-algebra $X=(X, \cdot, 0)$ is defined as follows:

$$
(\forall x, y \in X)(x \leqslant y \Leftrightarrow x \cdot y=0)
$$

and the following assertions are valid (see $[10,11])$.

$$
\begin{aligned}
& (\forall x \in X)(x \leqslant x) \\
& (\forall x, y, z \in X)(x \leqslant y, y \leqslant z \Rightarrow x \leqslant z) \\
& (\forall x, y, z \in X)(x \leqslant y \Rightarrow z \cdot x \leqslant z \cdot y) \\
& (\forall x, y, z \in X)(x \leqslant y \Rightarrow y \cdot z \leqslant x \cdot z) \\
& (\forall x, y, z \in X)(x \leqslant y \cdot x, \text { in particular, } y \cdot z \leqslant x \cdot(y \cdot z)) \\
& (\forall x, y \in X)(y \cdot x \leqslant x \Leftrightarrow x=y \cdot x) \\
& (\forall x, y \in X)(x \leqslant y \cdot y), \\
& (\forall a, x, y, z \in X)(x \cdot(y \cdot z) \leqslant x \cdot((a \cdot y) \cdot(a \cdot z))) \\
& (\forall a, x, y, z \in X)(((a \cdot x) \cdot(a \cdot y)) \cdot z \leqslant(x \cdot y) \cdot z) \\
& (\forall x, y, z \in X)((x \cdot y) \cdot z \leqslant y \cdot z) \\
& (\forall x, y, z \in X)(x \leqslant y \Rightarrow x \leqslant z \cdot y) \\
& (\forall x, y, z \in X)((x \cdot y) \cdot z \leqslant x \cdot(y \cdot z)), \text { and } \\
& (\forall a, x, y, z \in X)((x \cdot y) \cdot z \leqslant y \cdot(a \cdot z)) .
\end{aligned}
$$

Example 2.2 ([22]). Let $U$ be a nonempty set and let $X \in \mathcal{P}(U)$, where $\mathcal{P}(U)$ means the power set of $U$. Let $\mathcal{P}_{X}(U)=\{A \in \mathcal{P}(U) \mid X \subseteq A\}$. Define a binary operation $\triangle$ on $\mathcal{P}_{X}(U)$ by putting $A \triangle B=B \cap\left(A^{C} \cup X\right)$ for all $A, B \in \mathcal{P}_{X}(U)$, where $A^{C}$ means the complement of a subset $A$. Then $\left(\mathcal{P}_{X}(U), \triangle, X\right)$ is a UP-algebra. Let $\mathcal{P}^{X}(\mathrm{U})=\{\mathrm{A} \in \mathcal{P}(\mathrm{U}) \mid \mathrm{A} \subseteq \mathrm{X}\}$. Define a binary operation $\boldsymbol{\Delta}$ on $\mathcal{P}^{\mathrm{X}}(\mathrm{U})$ by putting $A \boldsymbol{\Delta} \mathrm{B}=\mathrm{B} \cup\left(\mathrm{A}^{\mathrm{C}} \cap \mathrm{X}\right)$ for all $A, B \in \mathcal{P}^{X}(U)$. Then $\left(\mathcal{P}^{X}(U), \mathbf{\Lambda}, X\right)$ is a UP-algebra.

Example 2.3 ([7]). Let $\mathbb{Z}^{*}$ be the set of all nonnegative integers. Define two binary operations $\circ$ and $\star$ on $\mathbb{Z}^{*}$ by:

$$
\left(\forall m, n \in \mathbb{Z}^{*}\right)\left(m \circ n=\left\{\begin{array}{ll}
n & \text { if } m<n, \\
0 & \text { otherwise }
\end{array}\right)\right.
$$

and

$$
\left(\forall m, n \in \mathbb{Z}^{*}\right)\left(m \star n=\left\{\begin{array}{ll}
n & \text { if } m>n \text { or } m=0 \\
0 & \text { otherwise }
\end{array}\right) .\right.
$$

Then $\left(\mathbb{Z}^{*}, \circ, 0\right)$ and $\left(\mathbb{Z}^{*}, \star, 0\right)$ are UP-algebras.

For more examples of UP-algebras, see [1, 2, 11, 14, 21-24].

For a nonempty subset $S$ of a UP-algebra $X=(X, \cdot, 0)$ which satisfies the following condition:

$$
(\forall x, y \in X)(y \in S \Rightarrow x \cdot y \in S) .
$$

Then the constant 0 of $X$ is in $S$. Indeed, let $x \in S$. By (2.3) and (2.5), we have $0=x \cdot x \in S$.

Definition $2.4([9,10,12,26])$. A nonempty subset $S$ of a UP-algebra $X=(X, \cdot, 0)$ is called

(1) a UP-subalgebra of $X$ if it satisfies the following condition:

$$
(\forall x, y \in S)(x \cdot y \in S)
$$


(2) a near UP-filter of $X$ if it satisfies the condition (2.5);

(3) a UP-filter of $X$ if it satisfies the following conditions:

$$
\begin{array}{r}
\text { the constant } 0 \text { of } X \text { is in } S \\
(\forall x, y \in X)(x \cdot y \in S, x \in S \Rightarrow y \in S)
\end{array}
$$

(4) an implicative UP-filter of $X$ if it satisfies the condition (2.6) and the following condition:

$$
(\forall x, y, z \in X)(x \cdot(y \cdot z) \in S, x \cdot y \in S \Rightarrow x \cdot z \in S) ;
$$

(5) a comparative UP-filter of $X$ if it satisfies the condition (2.6) and the following condition:

$$
(\forall x, y, z \in X)(x \cdot((y \cdot z) \cdot y) \in S, x \in S \Rightarrow y \in S) ;
$$

(6) a shift UP-filter of $X$ if it satisfies the condition (2.6) and the following condition:

$$
(\forall x, y, z \in X)(x \cdot(y \cdot z) \in S, x \in S \Rightarrow((z \cdot y) \cdot y) \cdot z \in S)
$$

(7) a UP-ideal of $X$ if it satisfies the condition (2.6) and the following condition:

$$
(\forall x, y, z \in X)(x \cdot(y \cdot z) \in S, y \in S \Rightarrow x \cdot z \in S) ;
$$

(8) a strong UP-ideal of $\mathrm{X}$ if it satisfies the condition (2.6) and the following condition:

$$
(\forall x, y, z \in X)((z \cdot y) \cdot(z \cdot x) \in S, y \in S \Rightarrow x \in S) .
$$

Guntasow et al. [9] proved that the only strong UP-ideal of a UP-algebra $X$ is $X$.

The following theorem is easy to verify.

Theorem 2.5. Let $\mathscr{U} \mathscr{F}$ be a nonempty family of UP-subalgebras (resp., near UP-filters, UP-filters, implicative UP-filters, comparative UP-filters, shift UP-filters, UP-ideals, strong UP-ideals) of a UP-algebra $\mathrm{X}=(\mathrm{X}, \cdot, 0)$. Then $\bigcap \mathscr{U} \mathscr{F}$ is a UP-subalgebra (resp., near UP-filter, UP-filter, implicative UP-filter, comparative UP-filter, shift UP-filter, UP-ideal, strong UP-ideal) of X.

\section{PFSs in UP-algebras by means of a special type}

In 2013, Cuong and Kreinovich [6] introduced the concept of picture fuzzy sets as the following definition.

A picture fuzzy set (briefly, PFS) in a nonempty set $X$ is a structure of the form:

$$
P=\left\{\left(x, r_{P}(x), g_{P}(x), b_{P}(x)\right) \mid x \in X\right\},
$$

where $r_{P}: X \rightarrow[0,1]$ is a positive membership, $g_{P}: X \rightarrow[0,1]$ is a neutral membership, and $b_{P}: X \rightarrow[0,1]$ is a negative membership satisfy the following condition:

$$
(\forall x \in X)\left(r_{\mathrm{P}}(x)+g_{\mathrm{P}}(x)+b_{\mathrm{P}}(x) \leqslant 1\right) .
$$

For our convenience, we will denote a PFS as $P=\left(X, r_{P}, g_{P}, b_{P}\right)=\left\{\left(x, r_{P}(x), g_{P}(x), b_{P}(x)\right) \mid x \in X\right\}$.

In what follows, let $X$ denote a UP-algebra $(X, \cdot, 0)$ unless otherwise specified.

Now, we introduce the eight new concepts of picture fuzzy sets in UP-algebras by means of a special type: special picture fuzzy UP-subalgebras, special picture fuzzy near UP-filters, special picture fuzzy UPfilters, special picture fuzzy implicative UP-filters, special picture fuzzy comparative UP-filters, special picture fuzzy shift UP-filters, special picture fuzzy UP-ideals, and special picture fuzzy strong UP-ideals, provide the necessary examples, investigate their properties, and prove their generalizations. 
Definition 3.1. A PFS $P$ in $X$ is called a special picture fuzzy UP-subalgebra of $X$ if it satisfies the following conditions:

$$
\begin{array}{r}
(\forall x, y \in X)\left(r_{P}(x \cdot y) \leqslant \max \left\{r_{P}(x), r_{P}(y)\right\}\right), \\
(\forall x, y \in X)\left(g_{P}(x \cdot y) \leqslant \max \left\{g_{P}(x), g_{P}(y)\right\}\right), \\
(\forall x, y \in X)\left(b_{P}(x \cdot y) \geqslant \min \left\{g_{P}(x), g_{P}(y)\right\}\right) .
\end{array}
$$

Example 3.2. Let $X=\{0,1,2,3,4\}$ be a UP-algebra with a fixed element 0 and a binary operation $\cdot$ defined by the following Cayley table:

\begin{tabular}{c|ccccc}
$\cdot$ & 0 & 1 & 2 & 3 & 4 \\
\hline 0 & 0 & 1 & 2 & 3 & 4 \\
1 & 0 & 0 & 2 & 3 & 4 \\
2 & 0 & 0 & 0 & 3 & 3 \\
3 & 0 & 1 & 2 & 0 & 3 \\
4 & 0 & 1 & 2 & 0 & 0
\end{tabular}

We define a PFS $P$ in $X$ as follows:

$$
r_{P}=\left(\begin{array}{ccccc}
0 & 1 & 2 & 3 & 4 \\
0.4 & 0.6 & 0.5 & 0.4 & 0.5
\end{array}\right), g P=\left(\begin{array}{ccccc}
0 & 1 & 2 & 3 & 4 \\
0.1 & 0.2 & 0.3 & 0.3 & 0.3
\end{array}\right), b_{P}=\left(\begin{array}{ccccc}
0 & 1 & 2 & 3 & 4 \\
0.2 & 0.1 & 0 & 0.1 & 0.2
\end{array}\right) .
$$

Hence, $P$ is a special picture fuzzy UP-subalgebra of $X$.

Definition 3.3. A PFS P in X is called a special picture fuzzy near UP-filter of $X$ if it satisfies the following conditions:

$$
\begin{aligned}
& (\forall x, y \in X)\left(r_{P}(x \cdot y) \leqslant r_{P}(y)\right) \\
& (\forall x, y \in X)\left(g_{P}(x \cdot y) \leqslant g_{P}(y)\right) \\
& (\forall x, y \in X)\left(b_{P}(x \cdot y) \geqslant b_{P}(y)\right) .
\end{aligned}
$$

Example 3.4. Let $X=\{0,1,2,3\}$ be a UP-algebra with a fixed element 0 and a binary operation - defined by the following Cayley table:

$$
\begin{array}{l|llll}
\cdot & 0 & 1 & 2 & 3 \\
\hline 0 & 0 & 1 & 2 & 3 \\
1 & 0 & 0 & 1 & 3 \\
2 & 0 & 0 & 0 & 3 \\
3 & 0 & 0 & 1 & 0
\end{array}
$$

We define a PFS $P$ in $X$ as follows:

$$
r_{P}=\left(\begin{array}{cccc}
0 & 1 & 2 & 3 \\
0.1 & 0.3 & 0.4 & 0.5
\end{array}\right), g_{P}=\left(\begin{array}{cccc}
0 & 1 & 2 & 3 \\
0.2 & 0.3 & 0.4 & 0.5
\end{array}\right), b_{P}=\left(\begin{array}{cccc}
0 & 1 & 2 & 3 \\
0.6 & 0.4 & 0.2 & 0
\end{array}\right) .
$$

Hence, $P$ is a special picture fuzzy near UP-filter of $X$.

Definition 3.5. A PFS $P$ in $X$ is called a special picture fuzzy UP-filter of $X$ if it satisfies the following conditions:

$$
\begin{aligned}
& (\forall x \in X)\left(r_{P}(0) \leqslant r_{P}(x)\right), \\
& (\forall x \in X)\left(g_{P}(0) \leqslant g_{P}(x)\right), \\
& (\forall x \in X)\left(b_{P}(0) \geqslant b_{P}(x)\right), \\
& (\forall x, y \in X)\left(r_{P}(y) \leqslant \max \left\{r_{P}(x \cdot y), r_{P}(x)\right\}\right), \\
& (\forall x, y \in X)\left(g_{P}(y) \leqslant \max \left\{g_{P}(x \cdot y), r_{P}(x)\right\}\right), \\
& (\forall x, y \in X)\left(b_{P}(y) \geqslant \min \left\{b_{P}(x \cdot y), b_{P}(x)\right\}\right) .
\end{aligned}
$$


Example 3.6. Let $X=\{0,1,2,3,4\}$ be a UP-algebra with a fixed element 0 and a binary operation $\cdot$ defined by the following Cayley table:

\begin{tabular}{c|ccccc}
$\cdot$ & 0 & 1 & 2 & 3 & 4 \\
\hline 0 & 0 & 1 & 2 & 3 & 4 \\
1 & 0 & 0 & 2 & 2 & 4 \\
2 & 0 & 0 & 0 & 2 & 4 \\
3 & 0 & 0 & 0 & 0 & 4 \\
4 & 0 & 1 & 2 & 3 & 0
\end{tabular}

We define a PFS $P$ in $X$ as follows:

$$
r_{P}=\left(\begin{array}{ccccc}
0 & 1 & 2 & 3 & 4 \\
0.1 & 0.1 & 0.2 & 0.2 & 0.2
\end{array}\right), g_{P}=\left(\begin{array}{ccccc}
0 & 1 & 2 & 3 & 4 \\
0 & 0 & 0.3 & 0.3 & 0.5
\end{array}\right), b_{P}=\left(\begin{array}{ccccc}
0 & 1 & 2 & 3 & 4 \\
0.6 & 0.5 & 0.5 & 0.5 & 0.3
\end{array}\right) .
$$

Hence, $P$ is a special picture fuzzy UP-filter of $X$.

Definition 3.7. A PFS $P$ in $X$ is called a special picture fuzzy implicative UP-filter of $X$ if it satisfies the following conditions: (3.7), (3.8), (3.9), and

$$
\begin{aligned}
& (\forall x, y, z \in X)\left(r_{\mathrm{P}}(x \cdot z) \leqslant \max \left\{\mathrm{r}_{\mathrm{P}}(x \cdot(y \cdot z)), \mathrm{r}_{\mathrm{P}}(x \cdot y)\right\}\right), \\
& (\forall x, y, z \in X)\left(\mathrm{g}_{\mathrm{P}}(x \cdot z) \leqslant \max \left\{g_{\mathrm{P}}(x \cdot(y \cdot z)), \mathrm{g}_{\mathrm{P}}(x \cdot y)\right\}\right), \\
& (\forall x, y, z \in X)\left(\mathrm{b}_{\mathrm{P}}(x \cdot z) \geqslant \min \left\{\mathrm{b}_{\mathrm{P}}(x \cdot(y \cdot z)), \mathrm{b}_{\mathrm{P}}(x \cdot y)\right\}\right) .
\end{aligned}
$$

Example 3.8. Let $X=\{0,1,2,3,4\}$ be a UP-algebra with a fixed element 0 and a binary operation $\cdot$ defined by the following Cayley table:

\begin{tabular}{c|ccccc}
$\cdot$ & 0 & 1 & 2 & 3 & 4 \\
\hline 0 & 0 & 1 & 2 & 3 & 4 \\
1 & 0 & 0 & 0 & 0 & 0 \\
2 & 0 & 1 & 0 & 0 & 0 \\
3 & 0 & 1 & 2 & 0 & 4 \\
4 & 0 & 1 & 2 & 3 & 0
\end{tabular}

We define a PFS $P$ in $X$ as follows:

$$
r_{P}=\left(\begin{array}{ccccc}
0 & 1 & 2 & 3 & 4 \\
0.4 & 0.5 & 0.5 & 0.5 & 0.5
\end{array}\right), g_{P}=\left(\begin{array}{ccccc}
0 & 1 & 2 & 3 & 4 \\
0.3 & 0.4 & 0.4 & 0.4 & 0.4
\end{array}\right), b_{P}=\left(\begin{array}{ccccc}
0 & 1 & 2 & 3 & 4 \\
0.2 & 0.1 & 0.1 & 0.1 & 0.1
\end{array}\right) .
$$

Hence, $P$ is a special picture fuzzy implicative UP-filter of $X$.

Definition 3.9. A PFS $P$ in $X$ is called a special picture fuzzy comparative UP-filter of $X$ if it satisfies the following conditions: (3.7), (3.8), (3.9), and

$$
\begin{aligned}
& (\forall x, y, z \in X)\left(r_{P}(y) \leqslant \max \left\{r_{P}(x \cdot((y \cdot z) \cdot y)), r_{P}(x)\right\}\right), \\
& (\forall x, y, z \in X)\left(g_{P}(y) \leqslant \max \left\{g_{P}(x \cdot((y \cdot z) \cdot y)), g_{P}(x)\right\}\right), \\
& (\forall x, y, z \in X)\left(b_{P}(y) \geqslant \min \left\{b_{P}(x \cdot((y \cdot z) \cdot y)), b_{P}(x)\right\}\right) .
\end{aligned}
$$

Example 3.10. Let $X=\{0,1,2,3,4\}$ be a UP-algebra with a fixed element 0 and a binary operation - defined by the following Cayley table:

\begin{tabular}{c|ccccc}
$\cdot$ & 0 & 1 & 2 & 3 & 4 \\
\hline 0 & 0 & 1 & 2 & 3 & 4 \\
1 & 0 & 0 & 1 & 2 & 4 \\
2 & 0 & 0 & 0 & 2 & 4 \\
3 & 0 & 0 & 0 & 0 & 4 \\
4 & 0 & 0 & 0 & 2 & 0
\end{tabular}


We define a PFS $P$ in $X$ as follows:

$$
r_{P}=\left(\begin{array}{ccccc}
0 & 1 & 2 & 3 & 4 \\
0.4 & 0.4 & 0.4 & 0.4 & 0.5
\end{array}\right), g_{P}=\left(\begin{array}{ccccc}
0 & 1 & 2 & 3 & 4 \\
0.3 & 0.3 & 0.3 & 0.3 & 0.4
\end{array}\right), b_{P}=\left(\begin{array}{ccccc}
0 & 1 & 2 & 3 & 4 \\
0.2 & 0.2 & 0.2 & 0.2 & 0.1
\end{array}\right) .
$$

Hence, $P$ is a special picture fuzzy comparative UP-filter of $X$.

Definition 3.11. A PFS $P$ in $X$ is called a special picture fuzzy shift UP-filter of $X$ if it satisfies the following conditions: (3.7), (3.8), (3.9), and

$$
\begin{aligned}
& (\forall x, y, z \in X)\left(\operatorname{r}_{\mathrm{P}}(((z \cdot y) \cdot y) \cdot z) \leqslant \max \left\{\mathrm{r}_{\mathrm{P}}(x \cdot(y \cdot z)), \mathrm{r}_{\mathrm{P}}(x)\right\}\right) \\
& (\forall x, y, z \in X)\left(g_{\mathrm{P}}(((z \cdot y) \cdot y) \cdot z) \leqslant \max \left\{g_{\mathrm{P}}(x \cdot(y \cdot z)), g_{\mathrm{P}}(x)\right\}\right) \\
& (\forall x, y, z \in X)\left(b_{\mathrm{P}}(((z \cdot y) \cdot y) \cdot z) \geqslant \min \left\{b_{\mathrm{P}}(x \cdot(y \cdot z)), b_{\mathrm{P}}(x)\right\}\right) .
\end{aligned}
$$

Example 3.12. Let $X=\{0,1,2,3,4\}$ be a UP-algebra with a fixed element 0 and a binary operation $\cdot$ defined by the following Cayley table:

\begin{tabular}{c|ccccc}
$\cdot$ & 0 & 1 & 2 & 3 & 4 \\
\hline 0 & 0 & 1 & 2 & 3 & 4 \\
1 & 0 & 0 & 1 & 2 & 4 \\
2 & 0 & 0 & 0 & 1 & 4 \\
3 & 0 & 0 & 0 & 0 & 4 \\
4 & 0 & 1 & 2 & 3 & 0
\end{tabular}

We define a PFS $P$ in $X$ as follows:

$$
r_{P}=\left(\begin{array}{ccccc}
0 & 1 & 2 & 3 & 4 \\
0.3 & 0.3 & 0.3 & 0.3 & 0.5
\end{array}\right), g_{P}=\left(\begin{array}{ccccc}
0 & 1 & 2 & 3 & 4 \\
0.2 & 0.2 & 0.2 & 0.2 & 0.4
\end{array}\right), b_{P}=\left(\begin{array}{ccccc}
0 & 1 & 2 & 3 & 4 \\
0.1 & 0.1 & 0.1 & 0.1 & 0
\end{array}\right) .
$$

Hence, $P$ is a special picture fuzzy shift UP-filter of $X$.

Definition 3.13. A PFS $P$ in $X$ is called a special picture fuzzy UP-ideal of $X$ if it satisfies the following conditions: (3.7), (3.8), (3.9), and

$$
\begin{aligned}
& (\forall x, y, z \in X)\left(\operatorname{r}_{\mathrm{P}}(x \cdot z) \leqslant \max \left\{\mathrm{r}_{\mathrm{P}}(x \cdot(y \cdot z)), \mathrm{r}_{\mathrm{P}}(y)\right\}\right), \\
& (\forall x, y, z \in X)\left(g_{\mathrm{P}}(x \cdot z) \leqslant \max \left\{\mathrm{g}_{\mathrm{P}}(x \cdot(y \cdot z)), g_{\mathrm{P}}(y)\right\}\right), \\
& (\forall x, y, z \in X)\left(\mathrm{b}_{\mathrm{P}}(x \cdot z) \geqslant \min \left\{\mathrm{b}_{\mathrm{P}}(x \cdot(y \cdot z)), \mathrm{b}_{\mathrm{P}}(y)\right\}\right) .
\end{aligned}
$$

Example 3.14. Let $X=\{0,1,2,3,4\}$ be a UP-algebra with a fixed element 0 and a binary operation $\cdot$ defined by the following Cayley table:

\begin{tabular}{c|ccccc}
$\cdot$ & 0 & 1 & 2 & 3 & 4 \\
\hline 0 & 0 & 1 & 2 & 3 & 4 \\
1 & 0 & 0 & 2 & 3 & 4 \\
2 & 0 & 0 & 0 & 3 & 3 \\
3 & 0 & 1 & 2 & 0 & 3 \\
4 & 0 & 1 & 2 & 0 & 0
\end{tabular}

We define a PFS $P$ in $X$ as follows:

$$
r_{P}=\left(\begin{array}{ccccc}
0 & 1 & 2 & 3 & 4 \\
0.1 & 0.1 & 0.3 & 0.3 & 0.3
\end{array}\right), g_{P}=\left(\begin{array}{ccccc}
0 & 1 & 2 & 3 & 4 \\
0 & 0 & 0.3 & 0.4 & 0.4
\end{array}\right), b_{P}=\left(\begin{array}{ccccc}
0 & 1 & 2 & 3 & 4 \\
0.6 & 0.6 & 0.3 & 0.3 & 0.3
\end{array}\right) .
$$

Hence, $P$ is a special picture fuzzy UP-ideal of $X$. 
Definition 3.15. A PFS $P$ in $X$ is called a special picture fuzzy strong UP-ideal of $X$ if it satisfies the following conditions: (3.7), (3.8), (3.9), and

$$
\begin{aligned}
& (\forall x, y, z \in X)\left(r_{P}(x) \leqslant \max \left\{r_{P}((z \cdot y) \cdot(z \cdot x)), r_{P}(y)\right\}\right), \\
& (\forall x, y, z \in X)\left(g_{P}(x) \leqslant \max \left\{g_{P}((z \cdot y) \cdot(z \cdot x)), g_{P}(y)\right\}\right), \\
& (\forall x, y, z \in X)\left(b_{P}(x) \geqslant \min \left\{b_{P}((z \cdot y) \cdot(z \cdot x)), b_{P}(y)\right\}\right) .
\end{aligned}
$$

Example 3.16. Let $X=\{0,1,2,3,4\}$ be a UP-algebra with a fixed element 0 and a binary operation - defined by the following Cayley table:

\begin{tabular}{c|ccccc}
$\cdot$ & 0 & 1 & 2 & 3 & 4 \\
\hline 0 & 0 & 1 & 2 & 3 & 4 \\
1 & 0 & 0 & 2 & 3 & 4 \\
2 & 0 & 1 & 0 & 2 & 4 \\
3 & 0 & 1 & 0 & 0 & 4 \\
4 & 0 & 1 & 0 & 3 & 0
\end{tabular}

We define a PFS $P$ in $X$ as follows:

$$
(\forall x \in X)\left(\begin{array}{l}
\mathrm{r}_{\mathrm{P}}(x)=0.3 \\
\mathrm{~g}_{\mathrm{P}}(x)=0.2 \\
\mathrm{~b}_{\mathrm{P}}(x)=0.4
\end{array}\right)
$$

Hence, $\mathrm{P}$ is a special picture fuzzy strong UP-ideal of $\mathrm{X}$.

Definition 3.17. A PFS $P$ in $X$ is said to be constant if $P$ is a constant function from $X$ to $[0,1]^{3}$. That is, $r_{P}, g_{P}$, and $b_{P}$ are constant functions from $X$ to $[0,1]$.

Theorem 3.18. Every special picture fuzzy UP-subalgebra of $X$ satisfies the conditions (3.7), (3.8), and (3.9).

Proof. Assume that $\mathrm{P}$ is a special picture fuzzy UP-subalgebra of $\mathrm{X}$. Let $\mathrm{x} \in \mathrm{X}$. Then

$$
\begin{aligned}
& r_{P}(0)=r_{P}(x \cdot x) \leqslant \max \left\{r_{P}(x), r_{P}(x)\right\}=r_{P}(x), \text { by }(2.3) \text { and }(3.1) \\
& g_{P}(0)=g_{P}(x \cdot x) \leqslant \max \left\{g_{P}(x), g_{P}(x)\right\}=g_{P}(x), \text { by }(2.3) \text { and }(3.2) \\
& b_{P}(0)=b_{P}(x \cdot x) \geqslant \min \left\{b_{P}(x), b_{P}(x)\right\}=b_{P}(x) . \text { by }(2.3) \text { and }(3.3)
\end{aligned}
$$

Hence, P satisfies the conditions (3.7), (3.8), and (3.9).

Theorem 3.19. A PFS $\mathrm{P}$ in $\mathrm{X}$ is constant if and only if it is a special picture fuzzy strong UP-ideal of $\mathrm{X}$.

Proof. Assume that $P$ is a PFS in $X$ which is constant. Then for all $x \in X, r_{P}(x)=r_{P}(0), g_{P}(x)=g_{P}(0)$, and $b_{P}(x)=b_{P}(0)$ and so $r_{P}(0) \leqslant r_{P}(x), g_{P}(0) \leqslant g_{P}(x)$, and $b_{P}(0) \geqslant b_{P}(x)$. Next, let $x, y, z \in X$. Then

$$
\begin{aligned}
& r_{P}(x) \leqslant r_{P}(0)=\max \left\{r_{P}(0), r_{P}(0)\right\}=\max \left\{r_{P}((z \cdot y) \cdot(z \cdot x)), r_{P}(y)\right\}, \\
& g_{P}(x) \leqslant g_{P}(0)=\max \left\{g_{P}(0), g_{P}(0)\right\}=\max \left\{g_{P}((z \cdot y) \cdot(z \cdot x)), g_{P}(y)\right\}, \\
& b_{P}(x) \geqslant b_{P}(0)=\min \left\{b_{P}(0), b_{P}(0)\right\}=\min \left\{b_{P}((z \cdot y) \cdot(z \cdot x)), b_{P}(y)\right\} .
\end{aligned}
$$

Hence, $P$ is a special picture fuzzy strong UP-ideal of $X$.

Conversely, assume that $P$ is a special picture fuzzy strong UP-ideal of $X$. Then for any $x \in X$,

$$
\begin{aligned}
\mathrm{r}_{\mathrm{P}}(x) & \leqslant \max \left\{\mathrm{r}_{\mathrm{P}}((x \cdot 0) \cdot(x \cdot x)), \mathrm{r}_{\mathrm{P}}(0)\right\} \text { by }(3.25) \\
& =\max \left\{\mathrm{r}_{\mathrm{P}}(0 \cdot(x \cdot x)), \mathrm{r}_{\mathrm{P}}(0)\right\} \text { by }(2.2) \\
& =\max \left\{\mathrm{r}_{\mathrm{P}}(x \cdot x), \mathrm{r}_{\mathrm{P}}(0)\right\} \text { by }(2.1) \\
& =\max \left\{\mathrm{r}_{\mathrm{P}}(0), \mathrm{r}_{\mathrm{P}}(0)\right\} \text { by }(2.3)
\end{aligned}
$$




$$
\begin{aligned}
& =r_{P}(0), \\
g_{P}(x) & \leqslant \max \left\{g_{P}((x \cdot 0) \cdot(x \cdot x)), g_{P}(0)\right\} \text { by }(3.26) \\
& =\max \left\{g_{P}(0 \cdot(x \cdot x)), g_{P}(0)\right\} \text { by }(2.2) \\
& =\max \left\{g_{P}(x \cdot x), g_{P}(0)\right\} \text { by }(2.1) \\
& =\max \left\{g_{P}(0), g_{P}(0)\right\} \text { by }(2.3) \\
& =g_{P}(0) \\
b_{P}(x) & \geqslant \min \left\{b_{P}((x \cdot 0) \cdot(x \cdot x)), b_{P}(0)\right\} \text { by }(3.27) \\
& =\min \left\{b_{P}(0 \cdot(x \cdot x)), b_{P}(0)\right\} \text { by }(2.2) \\
& =\min \left\{b_{P}(x \cdot x), b_{P}(0)\right\} \text { by }(2.1) \\
& =\min \left\{b_{P}(0), b_{P}(0)\right\} \text { by }(2.3) \\
& =b_{P}(0) .
\end{aligned}
$$

By (3.7), (3.8), and (3.9), we have $r_{P}(x)=r_{P}(0), g_{P}(x)=g_{P}(0)$, and $b_{P}(x)=b_{P}(0)$ for all $x \in X$. Hence, $P$ is constant.

Theorem 3.20. Every special picture fuzzy near UP-filter of $X$ is a special picture fuzzy UP-subalgebra.

Proof. Assume that $P$ is a special picture fuzzy near UP-filter of $X$. Let $x, y \in X$. Then

$$
\begin{aligned}
& r_{P}(x \cdot y) \leqslant r_{P}(y) \leqslant \max \left\{r_{P}(x), r_{P}(y)\right\} \text { by }(3.4) \\
& g_{P}(x \cdot y) \leqslant g_{P}(y) \leqslant \max \left\{g_{P}(x), g_{P}(y)\right\} \text { by (3.5), } \\
& b_{P}(x \cdot y) \geqslant b_{P}(y) \geqslant \min \left\{b_{P}(x), b_{P}(y)\right\} \text { by (3.6). }
\end{aligned}
$$

Hence, $P$ is a special picture fuzzy UP-subalgebra of $X$.

The following example shows that the converse of Theorem 3.20 is not true.

Example 3.21. From Example 3.2, we have $P$ is a special picture fuzzy UP-subalgebra of $X$. Since $b_{P}(3 \cdot 4)=$ $0.1<0.2=b_{\mathrm{P}}(4)$, we have $\mathrm{P}$ is not a special picture fuzzy near UP-filter of $\mathrm{X}$.

Theorem 3.22. Every special picture fuzzy near UP-filter of X satisfies the conditions (3.7), (3.8), and (3.9).

Proof. It is straightforward by Theorems 3.20 and 3.18 .

Theorem 3.23. Every special picture fuzzy UP-filter of $X$ is a special picture fuzzy near UP-filter.

Proof. Assume that $P$ is a special picture fuzzy UP-filter of $X$. Let $x, y \in X$. Then

$$
\begin{aligned}
r_{P}(x \cdot y) & \leqslant \max \left\{r_{P}(y \cdot(x \cdot y)), r_{P}(y)\right\} \text { by }(3.10) \\
& =\max \left\{r_{P}(0), r_{P}(y)\right\} \text { by }(2.4) \\
& =r_{P}(y) b y(3.7), \\
g_{P}(x \cdot y) & \leqslant \max \left\{g_{P}(y \cdot(x \cdot y)), g_{P}(y)\right\} \text { by }(3.11) \\
& =\max \left\{g_{P}(0), g_{P}(y)\right\} \text { by }(2.4) \\
& =g_{P}(y) b y(3.8), \\
b_{P}(x \cdot y) & \geqslant \min \left\{b_{P}(y \cdot(x \cdot y)), b_{P}(y)\right\} \text { by }(3.12) \\
& =\min \left\{b_{P}(0), b_{P}(y)\right\} \text { by }(2.4) \\
& =b_{P}(y) \text { by }(3.9) .
\end{aligned}
$$

Hence, $P$ is a special picture fuzzy near UP-filter of $X$.

The following example show that the converse of Theorem 3.23 is not true. 
Example 3.24. From Example 3.4, we have $P$ is a special picture fuzzy near UP-filter of $X$. Since $r_{P}(2)=$ $0.4>0.3=\max \left\{\mathrm{r}_{\mathrm{P}}(1 \cdot 2), \mathrm{r}_{\mathrm{P}}(1)\right\}$, we have $\mathrm{P}$ is not a special picture fuzzy UP-filter of $X$.

Theorem 3.25. Every special picture fuzzy UP-ideal of $X$ is a special picture fuzzy UP-filter.

Proof. Assume that $\mathrm{P}$ is a special picture fuzzy UP-ideal of $\mathrm{X}$. Then $\mathrm{P}$ satisfies the conditions (3.7), (3.8), and (3.9). Next, let $x, y \in X$. Then

$$
\begin{aligned}
r_{P}(y) & =r_{P}(0 \cdot y) \text { by }(2.1) \\
& \leqslant \max \left\{r_{P}(0 \cdot(x \cdot y)), r_{P}(x)\right\} \text { by }(3.22) \\
& =\max \left\{r_{P}(x \cdot y), r_{P}(x)\right\} \text { by }(2.1) \\
g_{P}(y) & =g_{P}(0 \cdot y) \text { by }(2.1) \\
& \leqslant \max \left\{g_{P}(0 \cdot(x \cdot y)), g_{P}(x)\right\} \text { by }(3.23) \\
& =\max \left\{g_{P}(x \cdot y), g_{P}(x)\right\} \text { by }(2.1) \\
b_{P}(y) & =b_{P}(0 \cdot y) b y(2.1) \\
& \geqslant \min \left\{b_{P}(0 \cdot(x \cdot y)), b_{P}(x)\right\} \text { by }(3.24) \\
& =\min \left\{b_{P}(x \cdot y), b_{P}(x)\right\} \text { by }(2.1) .
\end{aligned}
$$

Hence, $P$ is a special picture fuzzy UP-filter of $X$.

The following example show that the converse of Theorem 3.25 is not true.

Example 3.26. From Example 3.6, we have $P$ is a special picture fuzzy UP-filter of $X$. Since $r_{P}(2 \cdot 3)=0.2>$ $0.1=\max \left\{\mathrm{r}_{\mathrm{P}}(2 \cdot(1 \cdot 3)), \mathrm{r}_{\mathrm{P}}(1)\right\}$, we have $\mathrm{P}$ is not a special picture fuzzy UP-ideal of $\mathrm{X}$.

Theorem 3.27. Every special picture fuzzy implicative UP-filter of $X$ is a special picture fuzzy UP-filter.

Proof. Assume that $\mathrm{P}$ is a special picture fuzzy implicative UP-filter of $\mathrm{X}$. Then $\mathrm{P}$ satisfies the conditions (3.7), (3.8), and (3.9). Next, let $x, y \in X$. Then

$$
\begin{aligned}
r_{P}(y) & =r_{P}(0 \cdot y) \text { by }(2.1) \\
& \leqslant \max \left\{r_{P}(0 \cdot(x \cdot y)), r_{P}(0 \cdot x)\right\} \text { by }(3.13) \\
& =\max \left\{r_{P}(x \cdot y), r_{P}(x)\right\} \text { by }(2.1), \\
g_{P}(y) & =g_{P}(0 \cdot y) \text { by }(2.1) \\
& \leqslant \max \left\{g_{P}(0 \cdot(x \cdot y)), g_{P}(0 \cdot x)\right\} \text { by }(3.14) \\
& =\max \left\{g_{P}(x \cdot y), g_{P}(x)\right\} \text { by }(2.1), \\
b_{P}(y) & =b_{P}(0 \cdot y) b y(2.1) \\
& \geqslant \min \left\{b_{P}(0 \cdot(x \cdot y)), b_{P}(0 \cdot x)\right\} \text { by }(3.15) \\
& =\min \left\{b_{P}(x \cdot y), b_{P}(x)\right\} \text { by }(2.1) .
\end{aligned}
$$

Hence, $P$ is a special picture fuzzy UP-filter of $X$.

The following example show that the converse of Theorem 3.27 is not true.

Example 3.28. From Example 3.6, we have $P$ is a special picture fuzzy UP-filter of $X$. Since $r_{P}(2 \cdot 3)=0.2>$ $0.1=\max \left\{\mathrm{r}_{\mathrm{P}}(2 \cdot(1 \cdot 3)), \mathrm{r}_{\mathrm{P}}(2 \cdot 1)\right\}$, we have $\mathrm{P}$ is not a special picture fuzzy implicative UP-filter of $\mathrm{X}$.

Theorem 3.29. Every special picture fuzzy implicative UP-filter of $X$ is a special picture fuzzy UP-ideal.

Proof. Assume that $\mathrm{P}$ is a special picture fuzzy implicative UP-filter of $\mathrm{X}$. Then $\mathrm{P}$ satisfies the conditions (3.7), (3.8), and (3.9). Next, let $x, y, z \in X$. Then

$$
\mathrm{r}_{\mathrm{P}}(x \cdot z) \leqslant \max \left\{\mathrm{r}_{\mathrm{P}}(x \cdot(y \cdot z)), \mathrm{r}_{\mathrm{P}}(x \cdot y)\right\} \text { by (3.13) }
$$




$$
\begin{aligned}
& \leqslant \max \left\{r_{P}(x \cdot(y \cdot z)), r_{P}(y)\right\} \text { by Theorems } 3.27 \text { and } 3.23 \text { and (3.4), } \\
g_{P}(x \cdot z) & \leqslant \max \left\{g_{P}(x \cdot(y \cdot z)), g_{P}(x \cdot y)\right\} \text { by (3.14) } \\
& \leqslant \max \left\{g_{P}(x \cdot(y \cdot z)), g_{P}(y)\right\} \text { by Theorems } 3.27 \text { and } 3.23 \text { and (3.5), } \\
b_{P}(x \cdot z) & \geqslant \min \left\{b_{P}(x \cdot(y \cdot z)), b_{P}(x \cdot y)\right\} \text { by (3.15) } \\
& \geqslant \min \left\{b_{P}(x \cdot(y \cdot z)), b_{P}(y)\right\} \text { by Theorems } 3.27 \text { and } 3.23 \text { and (3.6). }
\end{aligned}
$$

Hence, $P$ is a special picture fuzzy UP-ideal of $X$.

The following example show that the converse of Theorem 3.29 is not true.

Example 3.30. From Example 3.14, we have $\mathrm{P}$ is a special picture fuzzy UP-ideal of $\mathrm{X}$. Since $\mathrm{g}_{\mathrm{P}}(3 \cdot 4)=$ $0.4>0=\max \left\{g_{\mathrm{P}}(3 \cdot(3 \cdot 4)), \mathrm{g}_{\mathrm{P}}(3 \cdot 3)\right\}$, we have $\mathrm{P}$ is not a special picture fuzzy implicative UP-filter of $\mathrm{X}$.

Theorem 3.31. Every special picture fuzzy comparative UP-filter of $X$ is a special picture fuzzy UP-filter.

Proof. Assume that $\mathrm{P}$ is a special picture fuzzy comparative UP-filter of $\mathrm{X}$. Then $\mathrm{P}$ satisfies the conditions (3.7), (3.8), and (3.9). Next, let $x, y \in X$. Then

$$
\begin{aligned}
r_{P}(y) & \leqslant \max \left\{r_{P}(x \cdot((y \cdot y) \cdot y)), r_{P}(x)\right\} \text { by }(3.16) \\
& =\max \left\{r_{P}(x \cdot(0 \cdot y)), r_{P}(x)\right\} \text { by }(2.3) \\
& =\max \left\{r_{P}(x \cdot y), r_{P}(x)\right\} \text { by }(2.1) . \\
g_{P}(y) & \leqslant \max \left\{g_{P}(x \cdot((y \cdot y) \cdot y)), g_{P}(x)\right\} \text { by }(3.17) \\
& =\max \left\{g_{P}(x \cdot(0 \cdot y)), g_{P}(x)\right\} \text { by }(2.3) \\
& =\max \left\{g_{P}(x \cdot y), g_{P}(x)\right\} \text { by }(2.1) \\
b_{P}(y) & \geqslant \min \left\{b_{P}(x \cdot((y \cdot y) \cdot y)), b_{P}(x)\right\} \text { by }(3.18) \\
& =\min \left\{b_{P}(x \cdot(0 \cdot y)), b_{P}(x)\right\} \text { by }(2.3) \\
& =\min \left\{b_{P}(x \cdot y), b_{P}(x)\right\} \text { by }(2.1) .
\end{aligned}
$$

Hence, $\mathrm{P}$ is a special picture fuzzy UP-filter of $\mathrm{X}$.

The following example show that the converse of Theorem 3.31 is not true.

Example 3.32. From Example 3.6, we have $P$ is a special picture fuzzy UP-filter of $X$. Since $b_{P}(2)=0.5<$ $0.6=\min \left\{b_{\mathrm{P}}(0 \cdot((2 \cdot 3) \cdot 2)), b_{\mathrm{P}}(0)\right\}$, we have $\mathrm{P}$ is not a special picture fuzzy comparative UP-filter of $X$.

Theorem 3.33. Every special picture fuzzy shift UP-filter of $X$ is a special picture fuzzy UP-filter.

Proof. Assume that $\mathrm{P}$ is a special picture fuzzy shift UP-filter of $\mathrm{X}$. Then $\mathrm{P}$ satisfies the conditions (3.7), (3.8), and (3.9). Next, let $x, y \in X$. Then

$$
\begin{aligned}
r_{P}(y) & =r_{P}(((y \cdot 0) \cdot 0) \cdot y) \text { by }(2.1) \text { and }(2.2) \\
& \leqslant \max \left\{r_{P}(x \cdot(0 \cdot y)), r_{P}(x)\right\} \text { by }(3.19) \\
& =\max \left\{r_{P}(x \cdot y), r_{P}(x)\right\} \text { by }(2.1), \\
g_{P}(y) & =g_{P}(((y \cdot 0) \cdot 0) \cdot y) \text { by }(2.1) \text { and }(2.2) \\
& \leqslant \max \left\{g_{P}(x \cdot(0 \cdot y)), g_{P}(x)\right\} \text { by }(3.20) \\
& =\max \left\{g_{P}(x \cdot y), g_{P}(x)\right\} \text { by }(2.1), \\
b_{P}(y) & =b_{P}(((y \cdot 0) \cdot 0) \cdot y) \text { by }(2.1) \text { and }(2.2) \\
& \geqslant \min \left\{b_{P}(x \cdot(0 \cdot y)), b_{P}(x)\right\} \text { by }(3.21) \\
& =\min \left\{b_{P}(x \cdot y), b_{P}(x)\right\} \text { by }(2.1) .
\end{aligned}
$$

Hence, $P$ is a special picture fuzzy UP-filter of $X$. 
The following example show that the converse of Theorem 3.33 is not true.

Example 3.34. From Example 3.6, we have $P$ is a special picture fuzzy UP-filter of $X$. Since $b_{P}(((1 \cdot 0) \cdot 0)$. $1)=0.5<0.6=\min \left\{b_{P}(0 \cdot(2 \cdot 1)), b_{P}(0)\right\}$, we have $P$ is not a special picture fuzzy shift UP-filter of $X$.

Theorem 3.35. Every special picture fuzzy strong UP-ideal of $\mathrm{X}$ is a special picture fuzzy implicative UP-filter.

Proof. Assume that $\mathrm{P}$ is a special picture fuzzy strong UP-ideal of $\mathrm{X}$. Then $\mathrm{P}$ satisfies the conditions (3.7), (3.8), and (3.9). By Theorem 3.19, we have P is constant. Next, let $x, y, z \in X$. Then

$$
\begin{aligned}
& r_{P}(x \cdot z)=r_{P}(x \cdot y) \text { by } r_{P} \text { is constant } \leqslant \max \left\{r_{P}(x \cdot(y \cdot z)), r_{P}(x \cdot y)\right\}, \\
& g_{P}(x \cdot z)=g_{P}(x \cdot y) \text { by } g_{P} \text { is constant } \leqslant \max \left\{g_{P}(x \cdot(y \cdot z)), g_{P}(x \cdot y)\right\}, \\
& b_{P}(x \cdot z)=b_{P}(x \cdot y) \text { by } b_{P} \text { is constant } \geqslant \min \left\{b_{P}(x \cdot(y \cdot z)), b_{P}(x \cdot y)\right\} .
\end{aligned}
$$

Hence, $\mathrm{P}$ is a special picture fuzzy implicative UP-filter of $\mathrm{X}$.

Theorem 3.36. Every special picture fuzzy strong UP-ideal of $X$ is a special picture fuzzy comparative UP-filter.

Proof. Assume that $\mathrm{P}$ is a special picture fuzzy strong UP-ideal of $X$. Then $\mathrm{P}$ satisfies the conditions (3.7), (3.8), and (3.9). By Theorem 3.19, we have P is constant. Next, let $x, y, z \in X$. Then

$$
\begin{aligned}
& r_{P}(y)=r_{P}(x) \text { by } r_{P} \text { is constant } \leqslant \max \left\{r_{P}(x \cdot((y \cdot z) \cdot y)), r_{P}(x)\right\}, \\
& g_{P}(y)=g_{P}(x) \text { by } g_{P} \text { is constant } \leqslant \max \left\{g_{P}(x \cdot((y \cdot z) \cdot y)), g_{P}(x)\right\}, \\
& b_{P}(y)=b_{P}(x) \text { by } b_{P} \text { is constant } \geqslant \min \left\{b_{P}(x \cdot((y \cdot z) \cdot y)), b_{P}(x)\right\} .
\end{aligned}
$$

Hence, $\mathrm{P}$ is a special picture fuzzy comparative UP-filter of $\mathrm{X}$.

Theorem 3.37. Every special picture fuzzy strong UP-ideal of $X$ is a special picture fuzzy shift UP-filter.

Proof. Assume that $\mathrm{P}$ is a special picture fuzzy strong UP-ideal of $X$. Then $\mathrm{P}$ satisfies the conditions (3.7), (3.8), and (3.9). By Theorem 3.19, we have P is constant. Next, let $x, y, z \in X$. Then

$$
\begin{aligned}
& r_{P}(((z \cdot y) \cdot y) \cdot z)=r_{P}(x) \text { by } r_{P} \text { is constant } \leqslant \max \left\{r_{P}(x \cdot(y \cdot z)), r_{P}(x)\right\}, \\
& g_{P}(((z \cdot y) \cdot y) \cdot z)=g_{P}(x) \text { by } g_{P} \text { is constant } \leqslant \max \left\{g_{P}(x \cdot(y \cdot z)), g_{P}(x)\right\}, \\
& b_{P}(((z \cdot y) \cdot y) \cdot z)=b_{P}(x) \text { by } b_{P} \text { is constant } \geqslant \min \left\{b_{P}(x \cdot(y \cdot z)), b_{P}(x)\right\} .
\end{aligned}
$$

Hence, $P$ is a special picture fuzzy shift UP-filter of $X$.

By Theorems 3.20, 3.23, 3.25, 3.27, 3.29, 3.31, 3.33, 3.35, 3.36, and 3.37 and Examples 3.21, 3.24, 3.26, $3.28,3.30,3.32$, and 3.34 , we have that the concept of special picture fuzzy UP-subalgebras is a generalization of special picture fuzzy near UP-filters, special picture fuzzy near UP-filters is a generalization of special picture fuzzy UP-filters, special picture fuzzy UP-filters is a generalization of special picture fuzzy comparative UP-filters, special picture fuzzy UP-filters is a generalization of special picture fuzzy shift UP-filters, special picture fuzzy UP-filters is a generalization of special picture fuzzy UP-ideals, special picture fuzzy UP-ideals is a generalization of special picture fuzzy implicative UP-filters, and special picture fuzzy implicative UP-filters, special picture fuzzy comparative UP-filters, and special picture fuzzy shift UP-filters is a generalization of special picture fuzzy strong UP-ideals. Moreover, by Theorem 3.19, we obtain that special picture fuzzy strong UP-ideals and constant picture fuzzy sets coincide.

Theorem 3.38. If $\mathrm{P}$ is a special picture fuzzy UP-subalgebra of $\mathrm{X}$ satisfying the following condition:

$$
(\forall x, y \in X)\left(x \cdot y \neq 0 \Rightarrow\left\{\begin{array}{l}
r_{P}(x) \leqslant r_{P}(y) \\
g_{P}(x) \leqslant g_{P}(y) \\
b_{P}(x) \geqslant b_{P}(y)
\end{array}\right),\right.
$$

then $\mathrm{P}$ is a special picture fuzzy near UP-filter of $\mathrm{X}$. 
Proof. Assume that $\mathrm{P}$ is a special picture fuzzy UP-subalgebra of $\mathrm{X}$ satisfying the condition (3.28). Let $x, y \in X$.

Case 1: $x \cdot y=0$. Then

$$
\begin{aligned}
& r_{P}(x \cdot y)=r_{P}(0) \leqslant r_{P}(y) \text { by (3.7) } \\
& g_{P}(x \cdot y)=g_{P}(0) \leqslant g_{P}(y) \text { by (3.8), } \\
& b_{P}(x \cdot y)=b_{P}(0) \geqslant b_{P}(y) \text { by (3.9). }
\end{aligned}
$$

Case 2: $x \cdot y \neq 0$. Then

$$
\begin{aligned}
& r_{P}(x \cdot y) \leqslant \max \left\{r_{P}(x), r_{P}(y)\right\}=r_{P}(y) \text { by (3.1) and (3.28) for } r_{P}, \\
& g_{P}(x \cdot y) \leqslant \max \left\{g_{P}(x), g_{P}(y)\right\}=g_{P}(y) \text { by (3.2) and (3.28) for } g_{P}, \\
& b_{P}(x \cdot y) \geqslant \min \left\{b_{P}(x), b_{P}(y)\right\}=b_{P}(y) \text { by (3.3) and (3.28) for } b_{P} .
\end{aligned}
$$

Hence, $P$ is a special picture fuzzy near UP-filter of $X$.

Theorem 3.39. If $\mathrm{P}$ is a special picture fuzzy near UP-filter of $\mathrm{X}$ satisfying the following condition:

$$
(\forall x \in X)\left(\begin{array}{c}
r_{P}(0)=g_{P}(0)=b_{P}(0) \\
r_{P}(x) \leqslant b_{P}(x) \\
g_{P}(x) \leqslant b_{P}(x)
\end{array}\right)
$$

then $\mathrm{P}$ is a special picture fuzzy strong UP-ideal of $\mathrm{X}$.

Proof. Assume that $P$ is a special picture fuzzy near UP-filter of $X$ satisfying the condition (3.29). By Theorem 3.22, we have P satisfies the conditions (3.7), (3.8), and (3.9). Next, let $x \in X$.

$$
\begin{aligned}
& r_{P}(0) \leqslant r_{P}(x) \leqslant b_{P}(x) \leqslant b_{P}(0)=r_{P}(0), \\
& g_{P}(0) \leqslant g_{P}(x) \leqslant b_{P}(x) \leqslant b_{P}(0)=g_{P}(0), \\
& b_{P}(0) \geqslant b_{P}(x) \geqslant r_{P}(x) \geqslant r_{P}(0)=b_{P}(0) .
\end{aligned}
$$

Thus $r_{P}(x)=r_{P}(0), g_{P}(x)=g_{P}(0)$, and $b_{P}(x)=b_{P}(0)$ for all $x \in X$, that is, $P$ is constant. By Theorem 3.19 , we have $P$ is a special picture fuzzy strong UP-ideal of $X$.

Theorem 3.40. If $\mathrm{P}$ is a special picture fuzzy UP-filter of $\mathrm{X}$ satisfying the following condition:

$$
(\forall x, y, z \in X)\left(\begin{array}{l}
r_{P}(y) \leqslant r_{P}(y \cdot(x \cdot z)) \Rightarrow r_{P}(y \cdot(x \cdot z)) \leqslant r_{P}(x \cdot(y \cdot z)) \\
g_{P}(y) \leqslant g_{P}(y \cdot(x \cdot z)) \Rightarrow g_{P}(y \cdot(x \cdot z)) \leqslant g_{P}(x \cdot(y \cdot z)) \\
b_{P}(y) \geqslant b_{P}(y \cdot(x \cdot z)) \Rightarrow b_{P}(y \cdot(x \cdot z)) \geqslant b_{P}(x \cdot(y \cdot z))
\end{array}\right),
$$

then $\mathrm{P}$ is a special picture fuzzy UP-ideal of $\mathrm{X}$.

Proof. Assume that $\mathrm{P}$ is a special picture fuzzy UP-filter of $X$ satisfying the condition (3.30). Then $P$ satisfies the conditions (3.7), (3.8), and (3.9). Next, let $x, y, z \in X$. Then

$$
\begin{aligned}
& r_{\mathrm{P}}(x \cdot z) \leqslant \max \left\{\mathrm{r}_{\mathrm{P}}(y \cdot(x \cdot z)), \mathrm{r}_{\mathrm{P}}(y)\right\} \text { by }(3.10) \leqslant \max \left\{\mathrm{r}_{\mathrm{P}}(x \cdot(y \cdot z)), \mathrm{r}_{\mathrm{P}}(\mathrm{y})\right\} \text { by (3.30) for } \mathrm{r}_{\mathrm{P}}, \\
& g_{P}(x \cdot z) \leqslant \max \left\{g_{P}(y \cdot(x \cdot z)), g_{P}(y)\right\} \text { by }(3.11) \leqslant \max \left\{g_{P}(x \cdot(y \cdot z)), g_{P}(y)\right\} \text { by (3.30) for } g_{P}, \\
& b_{\mathrm{P}}(x \cdot z) \geqslant \min \left\{b_{\mathrm{P}}(y \cdot(x \cdot z)), b_{\mathrm{P}}(y)\right\} \text { by }(3.12) \geqslant \min \left\{b_{\mathrm{P}}(x \cdot(y \cdot z)), b_{\mathrm{P}}(y)\right\} \text { by }(3.30) \text { for } b_{\mathrm{P}} \text {. }
\end{aligned}
$$

Hence, $P$ is a special picture fuzzy UP-ideal of $X$. 
Theorem 3.41. If $\mathrm{P}$ is a PFS in $\mathrm{X}$ satisfying the conditions (3.10), (3.11), and (3.12) and the following condition:

$$
(\forall x, y, z \in X)\left(\begin{array}{l}
r_{P}(x \cdot(y \cdot z)) \leqslant r_{P}((x \cdot(y \cdot z)) \cdot(x \cdot z)) \Rightarrow r_{P}((x \cdot(y \cdot z)) \cdot(x \cdot z)) \leqslant r_{P}(y) \\
g_{P}(x \cdot(y \cdot z)) \leqslant g_{P}((x \cdot(y \cdot z)) \cdot(x \cdot z)) \Rightarrow g_{P}((x \cdot(y \cdot z)) \cdot(x \cdot z)) \leqslant g_{P}(y) \\
b_{P}(x \cdot(y \cdot z)) \geqslant b_{P}((x \cdot(y \cdot z)) \cdot(x \cdot z)) \Rightarrow b_{P}((x \cdot(y \cdot z)) \cdot(x \cdot z)) \geqslant b_{P}(y)
\end{array}\right),
$$

then $\mathrm{P}$ is a special picture fuzzy UP-ideal of $\mathrm{X}$.

Proof. Assume that $\mathrm{P}$ is a PFS in X satisfying the conditions (3.10), (3.11), (3.12), and (3.31). If $z=0$ and by (3.31), we have $P$ satisfies the conditions (3.7), (3.8), and (3.9). Next, let $x, y, z \in X$. Then

$$
\begin{aligned}
r_{\mathrm{P}}(x \cdot z) & \leqslant \max \left\{\mathrm{r}_{\mathrm{P}}((x \cdot(y \cdot z)) \cdot(x \cdot z)), \mathrm{r}_{\mathrm{P}}(x \cdot(y \cdot z))\right\} \text { by }(3.10) \\
& \leqslant \max \left\{\mathrm{r}_{\mathrm{P}}(x \cdot(y \cdot z)), \mathrm{r}_{\mathrm{P}}(y)\right\} \text { by }(3.31) \text { for } \mathrm{r}_{\mathrm{P}}, \\
g_{\mathrm{P}}(x \cdot z) & \leqslant \max \left\{g_{\mathrm{P}}((x \cdot(y \cdot z)) \cdot(x \cdot z)), g_{\mathrm{P}}(x \cdot(y \cdot z))\right\} \text { by }(3.11) \\
& \leqslant \max \left\{g_{\mathrm{P}}(x \cdot(y \cdot z)), g_{\mathrm{P}}(y)\right\} \text { by }(3.31) \text { for } g_{\mathrm{P}}, \\
b_{\mathrm{P}}(x \cdot z) & \geqslant \min \left\{b_{\mathrm{P}}((x \cdot(y \cdot z)) \cdot(x \cdot z)), b_{\mathrm{P}}(x \cdot(y \cdot z))\right\} \text { by }(3.12) \\
& \geqslant \min \left\{\mathrm{b}_{\mathrm{P}}(x \cdot(y \cdot z)), \mathrm{b}_{\mathrm{P}}(y)\right\} \text { by }(3.31) \text { for } b_{\mathrm{P}} .
\end{aligned}
$$

Hence, $P$ is a special picture fuzzy UP-ideal of $X$.

Theorem 3.42. If $\mathrm{P}$ is a special picture fuzzy UP-ideal of $\mathrm{X}$ satisfying the following condition:

$$
(\forall x, y, z \in X)\left(\begin{array}{l}
r_{P}(x \cdot(y \cdot z)) \leqslant r_{P}(y) \Rightarrow r_{P}(y) \leqslant r_{P}(x \cdot y) \\
g_{P}(x \cdot(y \cdot z)) \leqslant g_{P}(y) \Rightarrow g_{P}(y) \leqslant g_{P}(x \cdot y) \\
b_{P}(x \cdot(y \cdot z)) \geqslant b_{P}(y) \Rightarrow b_{P}(y) \geqslant b_{P}(x \cdot y)
\end{array}\right)
$$

then $\mathrm{P}$ is a special picture fuzzy implicative UP-filter of $\mathrm{X}$.

Proof. Assume that $\mathrm{P}$ is a special picture fuzzy UP-ideal of $X$ satisfying the condition (3.32). Then $P$ satisfies the conditions (3.7), (3.8), and (3.9). Next, let $x, y, z \in X$. Then

$$
\begin{aligned}
& r_{P}(x \cdot z) \leqslant \max \left\{r_{P}(x \cdot(y \cdot z)), r_{P}(y)\right\} \text { by }(3.22) \leqslant \max \left\{r_{P}(x \cdot(y \cdot z)), r_{P}(x \cdot y)\right\} \text { by (3.32) for } r_{P}, \\
& g_{P}(x \cdot z) \leqslant \max \left\{g_{P}(x \cdot(y \cdot z)), g_{P}(y)\right\} \text { by }(3.23) \leqslant \max \left\{g_{P}(x \cdot(y \cdot z)), g_{P}(x \cdot y)\right\} \text { by (3.32) for } g_{P}, \\
& b_{P}(x \cdot z) \geqslant \min \left\{b_{P}(x \cdot(y \cdot z)), b_{P}(y)\right\} \text { by }(3.24) \geqslant \min \left\{b_{P}(x \cdot(y \cdot z)), b_{P}(x \cdot y)\right\} \text { by (3.32) for } b_{P} .
\end{aligned}
$$

Hence, $\mathrm{P}$ is a special picture fuzzy implicative UP-filter of $\mathrm{X}$.

Theorem 3.43. If $\mathrm{P}$ is a picture fuzzy UP-ideal of $\mathrm{X}$ satisfying the following condition:

$$
(\forall x, y, z \in X)\left(\begin{array}{l}
r_{P}(x \cdot y) \leqslant r_{P}(x \cdot((x \cdot y) \cdot z)) \Rightarrow r_{P}(x \cdot((x \cdot y) \cdot z)) \leqslant r_{P}(x \cdot(y \cdot z)) \\
g_{P}(x \cdot y) \leqslant g_{P}(x \cdot((x \cdot y) \cdot z)) \Rightarrow g_{P}(x \cdot((x \cdot y) \cdot z)) \leqslant g_{P}(x \cdot(y \cdot z)) \\
b_{P}(x \cdot y) \geqslant b_{P}(x \cdot((x \cdot y) \cdot z)) \Rightarrow b_{P}(x \cdot((x \cdot y) \cdot z)) \geqslant b_{P}(x \cdot(y \cdot z))
\end{array}\right)
$$

then $\mathrm{P}$ is a special picture fuzzy implicative UP-filter of $\mathrm{X}$.

Proof. Assume that $\mathrm{P}$ is a special picture fuzzy UP-ideal of $X$ satisfying the condition (3.33). Then $\mathrm{P}$ satisfies the conditions (3.7), (3.8), and (3.9). Next, let $x, y, z \in X$. Then

$$
\begin{aligned}
& r_{P}(x \cdot z) \leqslant \max \left\{r_{P}(x \cdot((x \cdot y) \cdot z)), r_{P}(x \cdot y)\right\} \text { by }(3.22) \leqslant \max \left\{r_{P}(x \cdot(y \cdot z)), r_{P}(x \cdot y)\right\} \text { by (3.33) for } r_{P}, \\
& g_{P}(x \cdot z) \leqslant \max \left\{g_{P}(x \cdot((x \cdot y) \cdot z)), g_{P}(x \cdot y)\right\} \text { by }(3.23) \leqslant \max \left\{g_{P}(x \cdot(y \cdot z)), g_{P}(x \cdot y)\right\} \text { by (3.33) for } g_{P}, \\
& b_{P}(x \cdot z) \geqslant \min \left\{b_{P}(x \cdot((x \cdot y) \cdot z)), b_{P}(x \cdot y)\right\} \text { by }(3.24) \geqslant \min \left\{b_{P}(x \cdot(y \cdot z)), b_{P}(x \cdot y)\right\} \text { by (3.33) for } b_{P} .
\end{aligned}
$$

Hence, $P$ is a special picture fuzzy implicative UP-filter of $X$. 
Theorem 3.44. If $\mathrm{P}$ is a special picture fuzzy UP-filter of $\mathrm{X}$ satisfying the following condition:

$$
(\forall x, y, z \in X)\left(\begin{array}{l}
r_{P}(x) \leqslant r_{P}(x \cdot y) \Rightarrow r_{P}(x \cdot y) \leqslant r_{P}(x \cdot((y \cdot z) \cdot y)) \\
r_{P}(x) \leqslant r_{P}(x \cdot y) \Rightarrow g_{P}(x \cdot y) \leqslant g_{P}(x \cdot((y \cdot z) \cdot y)) \\
r_{P}(x) \geqslant r_{P}(x \cdot y) \Rightarrow b_{P}(x \cdot y) \geqslant b_{P}(x \cdot((y \cdot z) \cdot y))
\end{array}\right)
$$

then $\mathrm{P}$ is a special picture fuzzy comparative UP-filter of $\mathrm{X}$.

Proof. Assume that $P$ is a special picture fuzzy UP-filter of $X$ satisfying the condition (3.34). Then $P$ satisfies the conditions (3.7), (3.8), and (3.9). Next, let $x, y, z \in X$. Then

$$
\begin{aligned}
& r_{P}(y) \leqslant \max \left\{r_{P}(x \cdot y), r_{P}(x)\right\} \text { by }(3.10) \leqslant \max \left\{r_{P}(x \cdot((y \cdot z) \cdot y)), r_{P}(x)\right\} \text { by (3.34) for } r_{P}, \\
& g_{P}(y) \leqslant \max \left\{g_{P}(x \cdot y), g_{P}(x)\right\} \text { by }(3.11) \leqslant \max \left\{g_{P}(x \cdot((y \cdot z) \cdot y)), g_{P}(x)\right\} \text { by (3.34) for } g_{P}, \\
& b_{P}(y) \geqslant \min \left\{b_{P}(x \cdot y), b_{P}(x)\right\} \text { by }(3.12) \geqslant \min \left\{b_{P}(x \cdot((y \cdot z) \cdot y)), b_{P}(x)\right\} \text { by (3.34) for } b_{P} .
\end{aligned}
$$

Hence, $\mathrm{P}$ is a special picture fuzzy comparative UP-filter of $\mathrm{X}$.

Theorem 3.45. If $\mathrm{P}$ is a PFS in $\mathrm{X}$ satisfying the conditions (3.10), (3.11), and (3.12) and the following condition:

$$
(\forall x, y, z \in X)\left(\begin{array}{l}
r_{P}(x \cdot((y \cdot z) \cdot y)) \leqslant r_{P}((x \cdot((y \cdot z) \cdot y)) \cdot y) \Rightarrow r_{P}((x \cdot((y \cdot z) \cdot y)) \cdot y) \leqslant r_{P}(x) \\
r_{P}(x \cdot((y \cdot z) \cdot y)) \leqslant r_{P}((x \cdot((y \cdot z) \cdot y)) \cdot y) \Rightarrow g_{P}((x \cdot((y \cdot z) \cdot y)) \cdot y) \leqslant g_{P}(x) \\
r_{P}(x \cdot((y \cdot z) \cdot y)) \geqslant r_{P}((x \cdot((y \cdot z) \cdot y)) \cdot y) \Rightarrow b_{P}((x \cdot((y \cdot z) \cdot y)) \cdot y) \geqslant b_{P}(x)
\end{array}\right),
$$

then $\mathrm{P}$ is a special picture fuzzy comparative UP-filter of $\mathrm{X}$.

Proof. Assume that $P$ is a PFS in $X$ satisfying the conditions (3.10), (3.11), (3.12), and (3.35). If $y=0$ and by (3.35), we have $P$ satisfies the conditions (3.7), (3.8), and (3.9). Next, let $x, y, z \in X$. Then

$$
\begin{aligned}
r_{P}(y) & \leqslant \max \left\{r_{P}((x \cdot((y \cdot z) \cdot y)) \cdot y), r_{P}(x \cdot((y \cdot z) \cdot y))\right\} \text { by }(3.10) \\
& \leqslant \max \left\{r_{P}(x \cdot((y \cdot z) \cdot y)), r_{P}(x)\right\} \text { by }(3.35) \text { for } r_{P} \\
g_{P}(y) & \leqslant \max \left\{g_{P}((x \cdot((y \cdot z) \cdot y)) \cdot y), g_{P}(x \cdot((y \cdot z) \cdot y))\right\} \text { by }(3.11) \\
& \leqslant \max \left\{g_{P}(x \cdot((y \cdot z) \cdot y)), g_{P}(x)\right\} \text { by }(3.35) \text { for } g_{P}, \\
b_{P}(y) & \geqslant \min \left\{b_{P}((x \cdot((y \cdot z) \cdot y)) \cdot y), b_{P}(x \cdot((y \cdot z) \cdot y))\right\} \text { by }(3.12) \\
& \geqslant \min \left\{b_{P}(x \cdot((y \cdot z) \cdot y)), b_{P}(x)\right\} \text { by }(3.35) \text { for } b_{P} .
\end{aligned}
$$

Hence, $P$ is a special picture fuzzy comparative UP-filter of $X$.

Theorem 3.46. If $\mathrm{P}$ is a special picture fuzzy UP-filter of $\mathrm{X}$ satisfying the following condition:

$$
(\forall x, y, z \in X)\left(\begin{array}{l}
r_{\mathrm{P}}(x) \leqslant r_{\mathrm{P}}(x \cdot(((z \cdot y) \cdot y) \cdot z)) \Rightarrow r_{\mathrm{P}}(x \cdot(((z \cdot y) \cdot y) \cdot z)) \leqslant r_{\mathrm{P}}(x \cdot(y \cdot z)) \\
g_{\mathrm{P}}(x) \leqslant g_{\mathrm{P}}(x \cdot(((z \cdot y) \cdot y) \cdot z)) \Rightarrow g_{\mathrm{P}}(x \cdot(((z \cdot y) \cdot y) \cdot z)) \leqslant g_{\mathrm{P}}(x \cdot(y \cdot z)) \\
b_{\mathrm{P}}(x) \geqslant b_{\mathrm{P}}(x \cdot(((z \cdot y) \cdot y) \cdot z)) \Rightarrow b_{\mathrm{P}}(x \cdot(((z \cdot y) \cdot y) \cdot z)) \geqslant b_{\mathrm{P}}(x \cdot(y \cdot z))
\end{array}\right),
$$

then $\mathrm{P}$ is a special picture fuzzy shift UP-filter of $\mathrm{X}$.

Proof. Assume that $\mathrm{P}$ is a special picture fuzzy UP-filter of $X$ satisfying the condition (3.36). Then $P$ satisfies the conditions (3.7), (3.8), and (3.9). Next, let $x, y, z \in X$. Then

$$
\begin{aligned}
r_{P}(((z \cdot y) \cdot y) \cdot z) & \leqslant \max \left\{r_{P}(x \cdot(((z \cdot y) \cdot y) \cdot z)), r_{P}(x)\right\} \text { by }(3.10) \\
& \leqslant \max \left\{r_{P}(x \cdot(y \cdot z)), r_{P}(x)\right\} \text { by }(3.36) \text { for } r_{P} \\
g_{P}(((z \cdot y) \cdot y) \cdot z) & \leqslant \max \left\{g_{P}(x \cdot(((z \cdot y) \cdot y) \cdot z)), g_{P}(x)\right\} \text { by }(3.11) \\
& \leqslant \max \left\{g_{P}(x \cdot(y \cdot z)), g_{P}(x)\right\} \text { by }(3.36) \text { for } g_{P} \\
b_{P}(((z \cdot y) \cdot y) \cdot z) & \geqslant \min \left\{b_{P}(x \cdot(((z \cdot y) \cdot y) \cdot z)), b_{P}(x)\right\} \text { by }(3.12) \\
& \geqslant \min \left\{b_{P}(x \cdot(y \cdot z)), b_{P}(x)\right\} \text { by }(3.36) \text { for } b_{P} .
\end{aligned}
$$

Hence, $P$ is a special picture fuzzy shift UP-filter of $X$. 
Theorem 3.47. If $\mathrm{P}$ is a PFS in $\mathrm{X}$ satisfying the conditions (3.10), (3.11), and (3.12) and the following condition:

$$
(\forall x, y, z \in X)\left(\begin{array}{l}
r_{P}(x \cdot(y \cdot z)) \leqslant r_{P}\left(( x \cdot ( y \cdot z ) ) \cdot ( ( ( z \cdot y ) \cdot y ) \cdot z ) \Rightarrow r _ { P } \left((x \cdot(y \cdot z)) \cdot(((z \cdot y) \cdot y) \cdot z) \leqslant r_{P}(x)\right.\right. \\
g_{P}(x \cdot(y \cdot z)) \leqslant g_{P}\left(( x \cdot ( y \cdot z ) ) \cdot ( ( ( z \cdot y ) \cdot y ) \cdot z ) \Rightarrow g _ { P } \left((x \cdot(y \cdot z)) \cdot(((z \cdot y) \cdot y) \cdot z) \leqslant g_{P}(x)\right.\right. \\
b_{P}(x \cdot(y \cdot z)) \geqslant b_{P}\left(( x \cdot ( y \cdot z ) ) \cdot ( ( ( z \cdot y ) \cdot y ) \cdot z ) \Rightarrow b _ { P } \left((x \cdot(y \cdot z)) \cdot(((z \cdot y) \cdot y) \cdot z) \geqslant b_{P}(x)\right.\right.
\end{array}\right),
$$

then $\mathrm{P}$ is a special picture fuzzy shift UP-filter of $X$.

Proof. Assume that $\mathrm{P}$ is a PFS in X satisfying the conditions (3.10), (3.11), (3.12), and (3.37). If $z=0$ and by (3.37), we have $P$ satisfies the conditions (3.7), (3.8), and (3.9). Next, let $x, y, z \in X$. Then

$$
\begin{aligned}
r_{P}(((z \cdot y) \cdot y) \cdot z) & \leqslant \max \left\{r_{P}\left((x \cdot(y \cdot z)) \cdot(((z \cdot y) \cdot y) \cdot z), r_{P}(x \cdot(y \cdot z))\right\} \text { by }(3.10)\right. \\
& \leqslant \max \left\{r_{P}(x \cdot(y \cdot z)), r_{P}(x)\right\} \text { by }(3.37) \text { for } r_{P} \\
g_{P}(((z \cdot y) \cdot y) \cdot z) & \leqslant \max \left\{g_{P}((x \cdot(y \cdot z)) \cdot(((z \cdot y) \cdot y) \cdot z)), g_{P}(x \cdot(y \cdot z))\right\} \text { by }(3.11) \\
& \leqslant \max \left\{g_{P}(x \cdot(y \cdot z)), g_{P}(x)\right\} \text { by }(3.37) \text { for } g_{P} \\
b_{P}(((z \cdot y) \cdot y) \cdot z) & \geqslant \min \left\{b_{P}\left((x \cdot(y \cdot z)) \cdot(((z \cdot y) \cdot y) \cdot z), b_{P}(x \cdot(y \cdot z))\right\} \text { by }(3.12)\right. \\
& \geqslant \min \left\{b_{P}(x \cdot(y \cdot z)), b_{P}(x)\right\} \text { by }(3.37) \text { for } b_{P} .
\end{aligned}
$$

Hence, $P$ is a special picture fuzzy shift UP-filter of $X$.

Theorem 3.48. If $\mathrm{P}$ is a PFS in $\mathrm{X}$ satisfying the following condition:

$$
(\forall x, y, z \in X)\left(z \leqslant x \cdot y \Rightarrow\left\{\begin{array}{l}
r_{P}(z) \leqslant \max \left\{r_{P}(x), r_{P}(y)\right\} \\
g_{P}(z) \leqslant \max \left\{g_{P}(x), g_{P}(y)\right\} \\
b_{P}(z) \geqslant \min \left\{b_{P}(x), b_{P}(y)\right\}
\end{array}\right)\right.
$$

then $\mathrm{P}$ is a special picture fuzzy UP-subalgebra of $\mathrm{X}$.

Proof. Assume that $P$ is a PFS in $X$ satisfying the condition (3.38). Let $x, y \in X$. By (2.3), we have $(x \cdot y) \cdot(x \cdot y)=0$, that is, $x \cdot y \leqslant x \cdot y$. It follows from (3.38) that

$$
r_{P}(x \cdot y) \leqslant \max \left\{r_{P}(x), r_{P}(y)\right\}, \quad g_{P}(x \cdot y) \leqslant \max \left\{g_{P}(x), g_{P}(y)\right\}, \quad b_{P}(x \cdot y) \geqslant \min \left\{b_{P}(x), b_{P}(y)\right\} .
$$

Hence, $P$ is a special picture fuzzy UP-subalgebra of $X$.

Theorem 3.49. If $\mathrm{P}$ is a PFS in $\mathrm{X}$ satisfying the following condition:

$$
(\forall x, y, z \in X)\left(z \leqslant x \cdot y \Rightarrow\left\{\begin{array}{l}
r_{P}(y) \leqslant \max \left\{r_{P}(z), r_{P}(x)\right\} \\
g_{P}(y) \leqslant \max \left\{g_{P}(z), g_{P}(x)\right\} \\
b_{P}(y) \geqslant \min \left\{b_{P}(z), b_{P}(x)\right\}
\end{array}\right)\right.
$$

then $\mathrm{P}$ is a special picture fuzzy UP-filter of $\mathrm{X}$.

Proof. Assume that $\mathrm{P}$ is a PFS in $X$ satisfying the condition (3.39). Let $x \in X$. By (2.2), we have $x \cdot(x \cdot 0)=0$, that is, $x \leqslant x \cdot 0$. It follows from (3.39) that

$$
\begin{aligned}
& r_{P}(0) \leqslant \max \left\{r_{P}(x), r_{P}(x)\right\}=r_{P}(x), \\
& g_{P}(0) \leqslant \max \left\{g_{P}(x), g_{P}(x)\right\}=g_{P}(x), \\
& b_{P}(0) \geqslant \min \left\{b_{P}(x), b_{P}(x)\right\}=b_{P}(x) .
\end{aligned}
$$

Next, let $x, y \in X$. By (2.3), we have $(x \cdot y) \cdot(x \cdot y)=0$, that is, $x \cdot y \leqslant x \cdot y$. It follows from (3.39) that

$$
r_{P}(y) \leqslant \max \left\{r_{P}(x \cdot y), r_{P}(x)\right\}, \quad g_{P}(y) \leqslant \max \left\{g_{P}(x \cdot y), g_{P}(x)\right\}, \quad b_{P}(y) \geqslant \min \left\{b_{P}(x \cdot y), b_{P}(x)\right\} .
$$

Hence, $P$ is a special picture fuzzy UP-filter of $X$. 
Theorem 3.50. If $\mathrm{P}$ is a PFS in $\mathrm{X}$ satisfying the following condition:

$$
(\forall a, x, y, z \in X)\left(a \leqslant x \cdot(y \cdot z) \Rightarrow\left\{\begin{array}{l}
r_{P}(x \cdot z) \leqslant \max \left\{r_{P}(a), r_{P}(y)\right\} \\
g_{P}(x \cdot z) \leqslant \max \left\{g_{P}(a), g_{P}(y)\right\} \\
b_{P}(x \cdot z) \geqslant \min \left\{b_{P}(a), b_{P}(y)\right\}
\end{array}\right),\right.
$$

then $\mathrm{P}$ is a special picture fuzzy UP-ideal of $\mathrm{X}$.

Proof. Assume that $\mathrm{P}$ is a PFS in $\mathrm{X}$ satisfying the condition (3.40). Let $x \in X$. By (2.2), we have $x \cdot(0 \cdot(x$. $0))=0$, that is, $x \leqslant 0 \cdot(x \cdot 0)$. It follows from (3.40) that

$$
\begin{aligned}
& r_{\mathrm{P}}(0)=\mathrm{r}_{\mathrm{P}}(0 \cdot 0) \leqslant \max \left\{\mathrm{r}_{\mathrm{P}}(\mathrm{x}), \mathrm{r}_{\mathrm{P}}(\mathrm{x})\right\}=\mathrm{r}_{\mathrm{P}}(\mathrm{x}) \text { by }(2.1), \\
& g_{\mathrm{P}}(0)=g_{\mathrm{P}}(0 \cdot 0) \leqslant \max \left\{g_{\mathrm{P}}(x), g_{\mathrm{P}}(x)\right\}=g_{\mathrm{P}}(x) \text { by }(2.1), \\
& \mathrm{b}_{\mathrm{P}}(0)=\mathrm{b}_{\mathrm{P}}(0 \cdot 0) \geqslant \min \left\{\mathrm{b}_{\mathrm{P}}(\mathrm{x}), \mathrm{b}_{\mathrm{P}}(\mathrm{x})\right\}=\mathrm{b}_{\mathrm{P}}(\mathrm{x}) \text { by }(2.1) .
\end{aligned}
$$

Next, let $x, y, z \in X$. By (2.3), we have $(x \cdot(y \cdot z)) \cdot(x \cdot(y \cdot z))=0$, that is, $x \cdot(y \cdot z) \leqslant x \cdot(y \cdot z)$. It follows from (3.40) that

$$
\begin{aligned}
& \mathrm{r}_{\mathrm{P}}(x \cdot z) \leqslant \max \left\{\mathrm{r}_{\mathrm{P}}(x \cdot(y \cdot z)), \mathrm{r}_{\mathrm{P}}(y)\right\}, \\
& g_{\mathrm{P}}(x \cdot z) \leqslant \max \left\{g_{\mathrm{P}}(x \cdot(y \cdot z)), g_{\mathrm{P}}(y)\right\}, \\
& \mathrm{b}_{\mathrm{P}}(x \cdot z) \geqslant \min \left\{g_{\mathrm{P}}(x \cdot(y \cdot z)), g_{\mathrm{P}}(y)\right\} .
\end{aligned}
$$

Hence, $P$ is a special picture fuzzy UP-ideal of $X$.

Theorem 3.51. If $\mathrm{P}$ is a PFS in $\mathrm{X}$ satisfying the following condition:

$$
(\forall a, x, y, z \in X)\left(a \leqslant x \cdot(y \cdot z) \Rightarrow\left\{\begin{array}{l}
r_{P}(x \cdot z) \leqslant \max \left\{r_{P}(a), r_{P}(x \cdot y)\right\} \\
g_{P}(x \cdot z) \leqslant \max \left\{g_{P}(a), g_{P}(x \cdot y)\right\} \\
b_{P}(x \cdot z) \geqslant \min \left\{b_{P}(a), b_{P}(x \cdot y)\right\}
\end{array}\right),\right.
$$

then $\mathrm{P}$ is a special picture fuzzy implicative UP-filter of $\mathrm{X}$.

Proof. Assume that $P$ is a PFS in $X$ satisfying the condition (3.41). Let $x \in X$. By (2.2), we have $x \cdot(0 \cdot(x$. $0))=0$, that is, $x \leqslant 0 \cdot(x \cdot 0)$. It follows from (3.41) that

$$
\begin{aligned}
& r_{P}(0)=r_{P}(0 \cdot 0) \leqslant \max \left\{r_{P}(x), r_{P}(0 \cdot x)\right\}=\max \left\{r_{P}(x), r_{P}(x)\right\}=r_{P}(x) \text { by }(2.1), \\
& g_{P}(0)=g_{P}(0 \cdot 0) \leqslant \max \left\{g_{P}(x), g_{P}(0 \cdot x)\right\}=\max \left\{g_{P}(x), g_{P}(x)\right\}=g_{P}(x) \text { by }(2.1), \\
& b_{P}(0)=b_{P}(0 \cdot 0) \geqslant \min \left\{b_{P}(x), b_{P}(0 \cdot x)\right\}=\min \left\{b_{P}(x), b_{P}(x)\right\}=b_{P}(x) \text { by }(2.1) .
\end{aligned}
$$

Next, let $x, y, z \in X$. By (2.3), we have $(x \cdot(y \cdot z)) \cdot(x \cdot(y \cdot z))=0$, that is, $x \cdot(y \cdot z) \leqslant x \cdot(y \cdot z)$. It follows from (3.41) that

$$
\begin{aligned}
& r_{P}(x \cdot z) \leqslant \max \left\{r_{P}(x \cdot(y \cdot z)), r_{P}(x \cdot y)\right\} \\
& g_{P}(x \cdot z) \leqslant \max \left\{g_{P}(x \cdot(y \cdot z)), g_{P}(x \cdot y)\right\}, \\
& b_{P}(x \cdot z) \geqslant \min \left\{b_{P}(x \cdot(y \cdot z)), b_{P}(x \cdot y)\right\} .
\end{aligned}
$$

Hence, $P$ is a special picture fuzzy implicative UP-filter of $X$.

Theorem 3.52. If $\mathrm{P}$ is a PFS in $\mathrm{X}$ satisfying the following condition:

$$
(\forall a, x, y, z \in X)\left(a \leqslant x \cdot((y \cdot z) \cdot y) \Rightarrow\left\{\begin{array}{l}
r_{P}(y) \leqslant \max \left\{r_{P}(a), r_{P}(x)\right\} \\
g_{P}(y) \leqslant \max \left\{g_{P}(a), g_{P}(x)\right\} \\
b_{P}(y) \geqslant \min \left\{b_{P}(a), b_{P}(x)\right\}
\end{array}\right),\right.
$$

then $\mathrm{P}$ is a special picture fuzzy comparative UP-filter of $\mathrm{X}$. 
Proof. Assume that $P$ is a PFS in $X$ satisfying the condition (3.42). Let $x \in X$. By (2.2), we have $x \cdot(x \cdot((0$. $x) \cdot 0))=0$, that is, $x \leqslant x \cdot((0 \cdot x) \cdot 0)$. It follows from (3.42) that

$$
\begin{aligned}
& r_{P}(0) \leqslant \max \left\{r_{P}(x), r_{P}(x)\right\}=r_{P}(x), \\
& g_{P}(0) \leqslant \max \left\{g_{P}(x), g_{P}(x)\right\}=g_{P}(x), \\
& b_{P}(0) \geqslant \min \left\{b_{P}(x), b_{P}(x)\right\}=b_{P}(x) .
\end{aligned}
$$

Next, let $x, y, z \in X$. By $(2.3)$, we have $(x \cdot((y \cdot z) \cdot y)) \cdot(x \cdot((y \cdot z) \cdot y))=0$, that is, $x \cdot((y \cdot z) \cdot y) \leqslant$ $x \cdot((y \cdot z) \cdot y)$. It follows from $(3.42)$ that

$$
\begin{aligned}
& r_{P}(y) \leqslant \max \left\{r_{P}(x \cdot((y \cdot z) \cdot y)), r_{P}(x)\right\}, \\
& g_{P}(y) \leqslant \max \left\{g_{P}(x \cdot((y \cdot z) \cdot y)), g_{P}(x)\right\}, \\
& b_{P}(y) \geqslant \min \left\{b_{P}(x \cdot((y \cdot z) \cdot y)), b_{P}(x)\right\} .
\end{aligned}
$$

Hence, $\mathrm{P}$ is a special picture fuzzy comparative UP-filter of $\mathrm{X}$.

Theorem 3.53. If $\mathrm{P}$ is a PFS in $\mathrm{X}$ satisfying the following condition:

$$
(\forall a, x, y, z \in X)\left(a \leqslant x \cdot(y \cdot z) \Rightarrow\left\{\begin{array}{l}
r_{P}(((z \cdot y) \cdot y) \cdot z) \leqslant \max \left\{r_{P}(a), r_{P}(x)\right\} \\
g_{P}(((z \cdot y) \cdot y) \cdot z) \leqslant \max \left\{g_{P}(a), g_{P}(x)\right\} \\
b_{P}(((z \cdot y) \cdot y) \cdot z) \geqslant \min \left\{b_{P}(a), b_{P}(x)\right\}
\end{array}\right)\right.
$$

then $\mathrm{P}$ is a special picture fuzzy shift UP-filter of $\mathrm{X}$.

Proof. Assume that $P$ is a PFS in $X$ satisfying the condition (3.43). Let $x \in X$. By (2.2), we have $x \cdot(x \cdot(x$. $0))=0$, that is, $x \leqslant x \cdot(x \cdot 0)$. It follows from (3.43) that

$$
\begin{aligned}
& r_{P}(0)=r_{P}(((0 \cdot x) \cdot x) \cdot 0) \leqslant \max \left\{r_{P}(x), r_{P}(x)\right\}=r_{P}(x) \text { by }(2.2) \\
& g_{P}(0)=g_{P}(((0 \cdot x) \cdot x) \cdot 0) \leqslant \max \left\{g_{P}(x), g_{P}(x)\right\}=g_{P}(x) \text { by }(2.2), \\
& b_{P}(0)=b_{P}(((0 \cdot x) \cdot x) \cdot 0) \geqslant \min \left\{b_{P}(x), b_{P}(x)\right\}=b_{P}(x) \text { by }(2.2) .
\end{aligned}
$$

Next, let $x, y, z \in X$. By (2.3), we have $(x \cdot(y \cdot z)) \cdot(x \cdot(y \cdot z))=0$, that is, $x \cdot(y \cdot z) \leqslant x \cdot(y \cdot z)$. It follows from (3.43) that

$$
\begin{aligned}
& r_{P}(((z \cdot y) \cdot y) \cdot z) \leqslant \max \left\{r_{P}(x \cdot(y \cdot z)), r_{P}(x)\right\}, \\
& g_{P}(((z \cdot y) \cdot y) \cdot z) \leqslant \max \left\{g_{P}(x \cdot(y \cdot z)), g_{P}(x)\right\}, \\
& b_{P}(((z \cdot y) \cdot y) \cdot z) \geqslant \min \left\{b_{P}(x \cdot(y \cdot z)), b_{P}(x)\right\} .
\end{aligned}
$$

Hence, $P$ is a special picture fuzzy shift UP-filter of $X$.

Theorem 3.54. If $\mathrm{P}$ is a PFS in $\mathrm{X}$ satisfying the following condition:

$$
(\forall x, y, z \in X)\left(z \leqslant x \cdot y \Rightarrow\left\{\begin{array}{l}
r_{\mathrm{P}}(z) \leqslant r_{\mathrm{P}}(y) \\
g_{\mathrm{P}}(z) \leqslant g_{\mathrm{P}}(y) \\
b_{\mathrm{P}}(z) \geqslant \mathrm{b}_{\mathrm{P}}(y)
\end{array}\right)\right.
$$

then $\mathrm{P}$ is a special picture fuzzy strong UP-ideal of $\mathrm{X}$.

Proof. Assume that $P$ is a PFS in $X$ satisfying the condition (3.44). Let $x, y \in X$. By (2.2) and (2.3), we have $x \cdot(y \cdot y)=0$, that is, $x \leqslant y \cdot y$. It follows from (3.44) that $r_{P}(x) \leqslant r_{P}(y), g_{P}(x) \leqslant g_{P}(y)$, and $b_{P}(x) \geqslant b_{P}(y)$. Similarly, $r_{P}(y) \leqslant r_{P}(x), g_{P}(y) \leqslant g_{P}(x)$, and $b_{P}(y) \geqslant b_{P}(x)$. Then $r_{P}(x)=r_{P}(y), g_{P}(x)=g_{P}(y)$, and $b_{P}(x)=b_{P}(y)$. Thus $P$ is constant. By Theorem 3.19, we have $P$ is a special picture fuzzy strong UP-ideal of $X$. 
In this part, we define a picture fuzzy set in the same way as a characteristic function and study its characterizations from the related subset.

For any fixed numbers $r^{+}, r^{-}, g^{+}, g^{-}, b^{+}, b^{-} \in[0,1]$ such that $r^{+}>r^{-}, g^{+}>g^{-}, b^{+}>b^{-}$and a

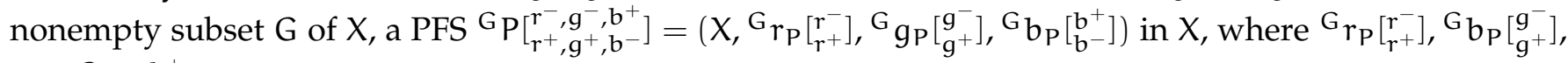
and ${ }^{G} b_{P}\left[b_{b^{-}}^{b^{+}}\right]$are functions on $X$ which are given as follows:

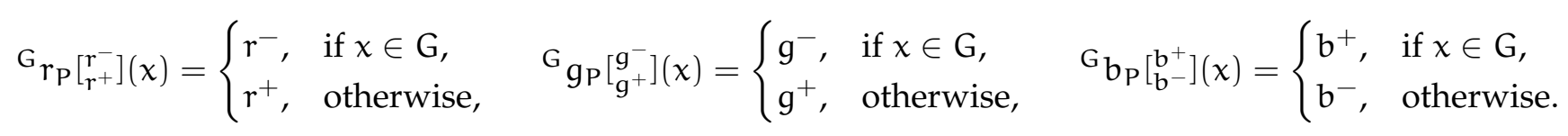

Lemma 3.55. If the constant 0 of $\mathrm{X}$ is in a nonempty subset $\mathrm{G}$ of $\mathrm{X}$, then the PFS ${ }^{\mathrm{G}} \mathrm{P}_{\mathrm{r}^{+}, \mathrm{g}^{+}, \mathrm{b}^{-}}^{\mathrm{r}^{-}} \mathrm{g}^{-}, \mathrm{b}^{+}$in $\mathrm{X}$ satisfies the conditions (3.7), (3.8), and (3.9).

Proof. If $0 \in \mathrm{G}$, then ${ }^{\mathrm{G}} \mathrm{r}_{\mathrm{P}}\left[\begin{array}{c}\mathrm{r}^{+} \\ \mathrm{r}^{-}\end{array}\right](0)=\mathrm{r}^{-},{ }^{\mathrm{G}} \mathrm{g}_{\mathrm{P}}\left[{ }_{\mathrm{g}^{+}}^{g^{-}}\right](0)=\mathrm{g}^{-}$, and ${ }^{\mathrm{G}} \mathrm{b}_{\mathrm{P}}\left[{ }_{\mathrm{b}^{-}}^{\mathrm{b}^{+}}\right](0)=\mathrm{b}^{+}$. Thus

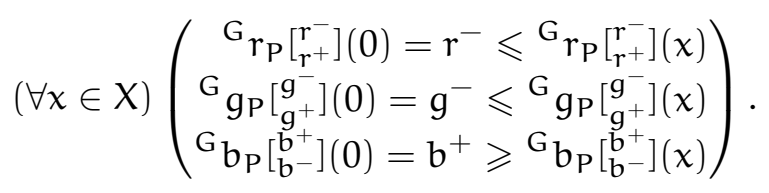

Hence, $\mathrm{P}^{\mathrm{P}}\left[\begin{array}{c}\mathrm{r}^{-}, \mathrm{g}^{-}, \mathrm{b}^{+} \\ \mathrm{r}^{+}, \mathrm{b}^{-}\end{array}\right]$satisfies the conditions (3.7), (3.8), and (3.9).

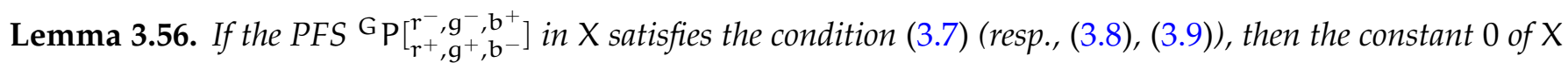
is in a nonempty subset $\mathrm{G}$ of $\mathrm{X}$.

Proof. Assume that the PFS ${ }^{G} P\left[\begin{array}{r}r_{r^{+}}^{-}, g^{+}, b^{-} \\ r^{+}\end{array}\right]$in $X$ satisfies the condition (3.7). Then $\left.{ }^{G} r_{P}{ }_{r^{+}}^{r^{-}}\right](0) \leqslant{ }^{G} r_{P}\left[\begin{array}{l}r_{r^{+}}^{-} \\ r^{+}\end{array}\right](x)$ for all $x \in X$. Since $G$ is nonempty, there exists $g \in G$. Thus ${ }^{G} r_{P}\left[\begin{array}{r}r^{-} \\ r^{+}\end{array}\right](g)=r^{-}$, so ${ }^{G} r_{P}\left[\begin{array}{r}r^{+} \\ r^{-}\end{array}\right](0) \leqslant{ }^{G} r_{P}\left[\begin{array}{r}r_{r}^{-} \\ r^{+}\end{array}\right](g)=r^{-}$, that is, ${ }^{G} r_{\mathrm{P}}\left[{ }_{r^{+}}^{r^{-}}\right](0)=r^{-}$. Hence, $0 \in \mathrm{G}$.

Theorem 3.57. The PFS GP $\mathrm{P}_{\mathrm{r}^{+}, \mathrm{g}^{+}, \mathrm{b}^{-}}^{\mathrm{r}^{-}}, \mathrm{g}^{-}, \mathrm{b}^{+} \mathrm{X} \mathrm{X}$ is a special picture fuzzy UP-subalgebra of $\mathrm{X}$ if and only if a nonempty subset $\mathrm{G}$ of $\mathrm{X}$ is a UP-subalgebra of $\mathrm{X}$.

Proof. Assume that ${ }^{G} \mathrm{P}_{r^{+}} r^{-}, g^{+}, b^{-}, b^{+}$is a special picture fuzzy UP-subalgebra of $X$. Let $x, y \in G$. Then $\left.{ }^{G} r_{P} r_{r^{+}}^{r^{-}}\right](x)=r^{-}={ }^{G} r_{P}\left[\begin{array}{r}r^{+} \\ r^{-}\end{array}\right](y)$. Thus

$$
{ }^{G} r_{P}\left[\begin{array}{r}
r^{+} \\
r^{-}
\end{array}\right](x \cdot y) \leqslant \max \left\{{ }^{G} r_{P}\left[r_{r^{+}}^{r^{-}}\right](x),{ }^{G} r_{P}\left[\begin{array}{r}
r^{+} \\
r^{-}
\end{array}\right](y)\right\}=\max \left\{r^{-}, r^{-}\right\}=r^{-} \leqslant{ }^{G} r_{P}\left[\begin{array}{r}
r^{-} \\
r^{-}
\end{array}\right](x \cdot y) \text { by (3.1) }
$$

and so ${ }^{G} r_{P}\left[r_{r+}^{r-}\right](x \cdot y)=r^{-}$. Thus $x \cdot y \in G$. Hence, $G$ is a UP-subalgebra of $X$. Conversely, assume that $G$ is a UP-subalgebra of $X$. Let $x, y \in X$.

Case 1: $x, y \in G$. Then

$$
{ }^{G} r_{P}\left[\begin{array}{c}
r_{r^{+}}^{+}
\end{array}\right](x)=r^{-}={ }^{G} r_{P}\left[\begin{array}{c}
r_{r}^{-} \\
r^{+}
\end{array}\right](y), \quad{ }^{G} g_{P}\left[\begin{array}{l}
g^{-} \\
g^{+}
\end{array}\right](x)=g^{-}={ }^{G} g_{P}\left[\begin{array}{l}
g^{-} \\
g^{+}
\end{array}\right](y), \quad{ }^{G} b_{P}\left[\begin{array}{l}
b^{+} \\
b^{-}
\end{array}\right](x)=b^{+}={ }^{G} b_{P}\left[\begin{array}{l}
b^{+} \\
b^{-}
\end{array}\right](y) .
$$

Thus

$$
\begin{aligned}
& \max \left\{{ }^{G} r_{\mathrm{P}}\left[{ }_{r^{+}}^{r^{-}}\right](x),{ }^{G} r_{P}\left[{ }_{r^{+}}^{r^{-}}\right](y)\right\}=\max \left\{r^{-}, r^{-}\right\}=r^{-}, \\
& \max \left\{{ }^{G} g_{P}\left[{ }_{g^{+}}^{g^{-}}\right](x),{ }^{G} g_{P}\left[{ }_{\mathbf{g}^{+}}^{g^{-}}\right](y)\right\}=\max \left\{g^{-}, g^{-}\right\}=g^{-}, \\
& \min \left\{{ }^{G} b_{P}\left[\begin{array}{l}
b^{+} \\
b^{-}
\end{array}\right](x),{ }^{G} b_{P}\left[{ }_{b^{-}}^{b^{+}}\right](y)\right\}=\min \left\{b^{+}, b^{+}\right\}=b^{+} .
\end{aligned}
$$

Since $G$ is a UP-subalgebra of $X$, we have $x \cdot y \in G$ and so ${ }^{G} r_{P}\left[r_{r^{+}}^{r^{-}}\right](x \cdot y)=r^{-},{ }^{G} g_{P}\left[g_{g^{+}}^{g^{-}}\right](x \cdot y)=g^{-}$, and ${ }^{G} b_{\mathrm{P}}\left[{ }_{b^{-}}^{b^{+}}\right](x \cdot y)=b^{+}$. Hence,

$$
{ }^{G} r_{P}\left[\begin{array}{r}
r^{-} \\
r^{+}
\end{array}\right](x \cdot y)=r^{-} \leqslant r^{-}=\max \left\{{ }^{G} r_{P}\left[\begin{array}{r}
r^{-} \\
r^{+}
\end{array}\right](x),{ }^{G} r_{P}\left[\begin{array}{r}
r^{+} \\
r^{-}
\end{array}\right](y)\right\}
$$




$$
\begin{aligned}
& { }^{G} g_{P}\left[\left[_{g^{+}}^{g^{-}}\right](x \cdot y)=g^{-} \leqslant g^{-}=\max \left\{{ }^{G} g_{P}\left[{ }_{g^{+}}^{g^{-}}\right](x),{ }^{G} g_{P}\left[{ }_{g^{+}}^{g^{-}}\right](y)\right\},\right. \\
& { }^{G} b_{P}\left[{ }_{b^{-}}^{b^{+}}\right](x \cdot y)=b^{+} \geqslant b^{+}=\min \left\{{ }^{G} b_{P}\left[{ }_{b^{-}}^{b^{+}}\right](x),{ }^{G} b_{P}\left[{ }_{b^{-}}^{b^{+}}\right](y)\right\} .
\end{aligned}
$$

Case 2: $x \notin \mathrm{G}$ or $\mathrm{y} \notin \mathrm{G}$. Then

$$
\begin{aligned}
& { }^{G} r_{P}\left[\begin{array}{l}
r_{r}^{-} \\
r^{+}
\end{array}\right](x)=r^{+} \text {or }{ }^{G} r_{P}\left[\begin{array}{l}
r_{r}^{-} \\
r^{+}
\end{array}\right](y)=r^{+}, \\
& { }^{G} g_{P}\left[\begin{array}{l}
g^{-} \\
g^{+}
\end{array}\right](x)=g^{+} \text {or }{ }^{G} g_{P}\left[\begin{array}{l}
g^{+} \\
g^{+}
\end{array}\right](y)=g^{+}, \\
& { }^{G} b_{P}\left[\begin{array}{l}
b_{b^{+}} \\
b^{+}
\end{array}\right](x)=b^{-} \text {or }{ }^{G} b_{P}\left[\begin{array}{l}
b^{+} \\
b^{-}
\end{array}\right](y)=b^{-} .
\end{aligned}
$$

Thus

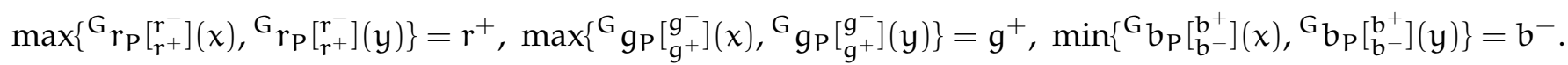

Therefore,

$$
\begin{aligned}
& { }^{G} r_{P}\left[r_{r^{+}}^{r^{-}}\right](x \cdot y) \leqslant r^{+}=\max \left\{{ }^{G} r_{P}\left[{ }_{r^{+}}^{r^{-}}\right](x),{ }^{G} r_{P}\left[r_{r^{+}}^{r^{-}}\right](y)\right\}, \\
& { }^{G} g_{P}\left[{ }_{g^{+}}^{g^{-}}\right](x \cdot y) \leqslant g^{+}=\max \left\{{ }^{G} g_{P}\left[{ }_{g^{+}}^{g^{-}}\right](x),{ }^{G} g_{P}\left[{ }_{g^{+}}^{g^{-}}\right](y)\right\}, \\
& { }^{G} b_{P}\left[{ }_{b^{-}}^{b^{+}}\right](x \cdot y) \geqslant b^{-}=\min \left\{{ }^{G} b_{P}\left[\begin{array}{l}
b^{+} \\
b^{-}
\end{array}\right](x),{ }^{G} b_{P}\left[b_{b^{-}}^{b^{+}}\right](y)\right\} \text {. }
\end{aligned}
$$

Hence, ${ }^{G} P\left[\begin{array}{c}r^{-}, g^{-}, b^{+} \\ r^{+}, g^{+}, b^{-}\end{array}\right]$is a special picture fuzzy UP-subalgebra of $X$.

Theorem 3.58. The PFS G $\mathrm{P}_{\mathrm{r}^{+}, \mathrm{g}^{+}, \mathrm{b}^{-}}^{\mathrm{r}^{-}, \mathrm{g}^{-}}$in $\mathrm{X}$ is a special picture fuzzy near UP-filter of $\mathrm{X}$ if and only if a nonempty subset $\mathrm{G}$ of $\mathrm{X}$ is a near UP-filter of $\mathrm{X}$.

Proof. Assume that $\mathrm{P}^{\mathrm{P}}\left[\begin{array}{c}\mathrm{r}^{-}, \mathrm{g}^{-}, \mathrm{b}^{+} \\ \mathrm{r}^{+}, \mathrm{b}^{-}\end{array}\right]$is special picture fuzzy near UP-filter of $X$. Let $x \in X$ and $y \in G$. Then ${ }^{G} r_{\mathrm{P}}\left[\begin{array}{c}r^{-} \\ r^{+}\end{array}\right](y)=r^{-}$. Thus

$$
{ }^{G} r_{P}\left[r_{r^{+}}^{r^{-}}\right](x \cdot y) \leqslant{ }^{G} r_{P}\left[\begin{array}{r}
r_{r}^{-} \\
r^{+}
\end{array}\right](y)=r^{-} \leqslant{ }^{G} r_{P}\left[\begin{array}{r}
r_{r}^{-} \\
r^{+}
\end{array}\right](x \cdot y) \text { by }(3.4)
$$

and so ${ }^{G} r_{P}\left[r_{r^{+}}^{r^{-}}\right](x \cdot y)=r^{-}$. Thus $x \cdot y \in G$. Hence, $G$ is a near UP-filter of $X$.

Conversely, assume that $G$ is a near UP-filter of $X$. Let $x, y \in X$.

Case 1: $y \in G$. Then ${ }^{G} r_{P}\left[\begin{array}{r}r^{+} \\ r^{-}\end{array}\right](y)=r^{-},{ }^{G} g_{P}\left[\begin{array}{c}g^{+} \\ g^{-}\end{array}\right](y)=g^{-}$, and ${ }^{G} b_{P}\left[b_{b}^{b^{+}}\right](y)=b^{+}$. Since $G$ is a near UP-filter of $X$, we have $x \cdot y \in G$ and so ${ }^{G} r_{P}\left[{ }_{r^{+}}^{r^{-}}\right](x \cdot y)=r^{-},{ }^{G} g_{P}\left[g^{+}{ }^{-}\right](x \cdot y)=g^{-}$, and ${ }^{G} b_{P}\left[{ }_{b^{-}}^{b^{+}}\right](x \cdot y)=b^{+}$. Thus

$$
\begin{aligned}
& { }^{G} r_{P}\left[\begin{array}{c}
r_{r^{-}}^{+}
\end{array}\right](x \cdot y)=r^{-} \leqslant r^{-}={ }^{G} r_{P}\left[\begin{array}{l}
r_{r^{+}}^{-} \\
r^{-}
\end{array}\right](y), \\
& { }^{G} g_{P}\left[\begin{array}{l}
g^{-} \\
g^{+}
\end{array}\right](x \cdot y)=g^{-} \leqslant g^{-}={ }^{G} g_{P}\left[\begin{array}{l}
g^{-} \\
g^{+}
\end{array}\right](y), \\
& { }^{G} b_{P}\left[{ }_{b^{+}}^{b^{+}}\right](x \cdot y)=b^{+} \geqslant b^{+}={ }^{G} b_{P}\left[\begin{array}{l}
b^{+} \\
b^{-}
\end{array}\right](y) .
\end{aligned}
$$

Case 2: $y \notin G$. Then ${ }^{G} r_{P}\left[\begin{array}{r}r^{+} \\ r^{+}\end{array}\right](y)=r^{+},{ }^{G} g_{P}\left[{ }_{g^{+}}^{g^{-}}\right](y)=g^{+}$, and ${ }^{G} b_{P}\left[{ }_{b^{-}}^{b^{+}}\right](y)=b^{-}$. Thus

$$
\begin{aligned}
& { }^{G} r_{P}\left[\begin{array}{r}
r^{-} \\
r^{-}
\end{array}\right](x \cdot y) \leqslant r^{+}={ }^{G} r_{P}\left[{ }_{r^{+}}^{r^{-}}\right](y), \\
& { }^{G} g_{P}\left[g_{g^{+}}^{g^{-}}\right](x \cdot y) \leqslant g^{+}={ }^{G} g_{P}\left[{ }_{g^{+}}^{g^{-}}\right](y) \text {, } \\
& { }^{G} b_{P}\left[\begin{array}{c}
b^{+} \\
b^{-}
\end{array}\right](x \cdot y) \geqslant b^{-}={ }^{G} b_{P}\left[\begin{array}{l}
b^{+} \\
b^{-}
\end{array}\right](y) \text {. }
\end{aligned}
$$

Hence, ${ }^{G} P\left[\begin{array}{c}r^{-}, g^{-}, \mathrm{g}^{+}, \mathrm{b}^{+} \\ \mathrm{r}^{+}\end{array}\right]$is a special picture fuzzy near UP-filter of $X$.

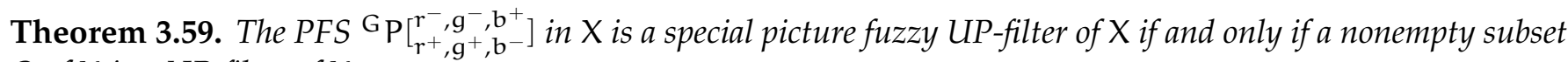
$\mathrm{G}$ of $\mathrm{X}$ is a UP-filter of $\mathrm{X}$. 
Proof. Assume that ${ }^{G} P\left[\begin{array}{c}r_{r}^{-}, g^{-}, b^{+} \\ r^{+}, b^{-}\end{array}\right]$is a special picture fuzzy UP-filter of $X$. Since ${ }^{G} P_{\left[r^{+}, g^{+}, b^{-}\right.}^{r^{-}},,^{-}, b^{+}$satisfies the condition (3.7), it follows from Lemma 3.56 that $0 \in G$. Next, let $x, y \in X$ be such that $x \cdot y \in G$ and $x \in G$. Then ${ }^{G} r_{P}\left[\begin{array}{r}r^{-} \\ r^{-}\end{array}\right](x \cdot y)=r^{-}={ }^{G} r_{P}\left[{ }_{r+}^{r^{-}}\right](x)$. Thus

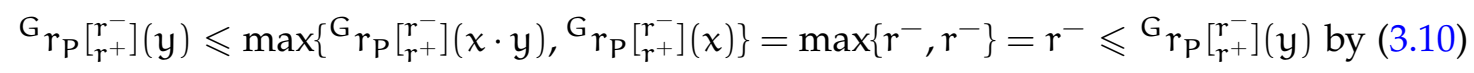

and so ${ }^{G} r_{P}\left[\begin{array}{r}r^{+} \\ r^{-}\end{array}\right](y)=r^{-}$. Thus $y \in G$. Hence, $G$ is a UP-filter of $X$.

Conversely, assume that $G$ is a UP-filter of $X$. Since $0 \in \mathrm{G}$, it follows from Lemma 3.55 that $\left.{ }^{G} \mathrm{P}_{\mathrm{r}^{+}}^{\mathrm{r}^{-}, \mathrm{g}^{+}, \mathrm{b}^{-}} \mathrm{b}^{+}\right]$ satisfies the conditions (3.7), (3.8), and (3.9). Next, let $x, y \in X$.

Case 1: $x \cdot y \in G$ and $x \in G$. Then

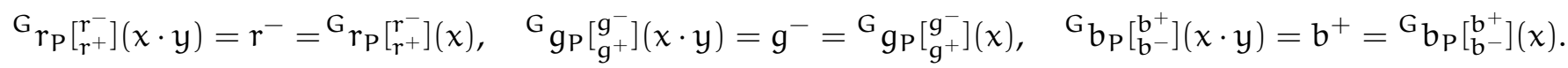

Thus

$$
\begin{aligned}
& \max \left\{{ }^{G} r_{\mathrm{P}}\left[r_{r^{+}}^{r^{-}}\right](x \cdot y),{ }^{G} r_{\mathrm{P}}\left[r_{r^{+}}^{r^{-}}\right](x)\right\}=\max \left\{r^{-}, r^{-}\right\}=r^{-}, \\
& \max \left\{{ }^{G} g_{P}\left[{ }_{g^{+}}^{g^{-}}\right](x \cdot y),{ }^{G} g_{P}\left[{ }_{g^{+}}^{g^{-}}\right](x)\right\}=\max \left\{g^{-}, g^{-}\right\}=g^{-} \text {, } \\
& \min \left\{{ }^{\mathrm{G}} \mathrm{b}_{\mathrm{P}}\left[\mathrm{b}^{\mathrm{b}^{+}}\right](\mathrm{x} \cdot \mathrm{y}),{ }^{\mathrm{G}} \mathrm{b}_{\mathrm{P}}\left[\mathrm{b}^{\mathrm{b}^{+}}\right](\mathrm{x})\right\}=\min \left\{\mathrm{b}^{+}, \mathrm{b}^{+}\right\}=\mathrm{b}^{+} \text {. }
\end{aligned}
$$

Since $G$ is a UP-filter of $X$, we have $y \in G$ and so ${ }^{G} r_{P}\left[\begin{array}{c}r^{-} \\ r^{+}\end{array}\right](y)=r^{-},{ }^{G} g_{P}\left[\begin{array}{l}g^{-} \\ g^{+}\end{array}\right](y)=g^{-}$, and ${ }^{G} b_{P}\left[b^{b^{+}}\right](y)=b^{+}$. Thus

$$
\begin{aligned}
& { }^{G} r_{\mathrm{P}}\left[\left[_{r^{-}}^{r^{-}}\right](y)=r^{-} \leqslant r^{-}=\max \left\{{ }^{G} r_{\mathrm{P}}\left[\begin{array}{c}
r_{r}^{-} \\
r^{+}
\end{array}\right](x \cdot y),{ }^{G} r_{\mathrm{P}}\left[\begin{array}{c}
r^{-} \\
r^{+}
\end{array}\right](x)\right\},\right. \\
& { }^{G} g_{P}\left[g_{g^{+}}^{g^{-}}\right](y)=g^{-} \leqslant g^{-}=\max \left\{{ }^{G} g_{P}\left[g_{g^{+}}^{g^{-}}\right](x \cdot y),{ }^{G} g_{P}\left[g_{g^{+}}^{g^{-}}\right](x)\right\}, \\
& { }^{G} b_{P}\left[b_{b^{-}}^{b^{+}}\right](y)=b^{+} \geqslant b^{+}=\min \left\{{ }^{G} b_{P}\left[b^{b^{+}}\right](x \cdot y),{ }^{G} b_{P}\left[b_{b^{-}}^{b^{+}}\right](x)\right\} \text {. }
\end{aligned}
$$

Case 2: $x \cdot y \notin G$ or $x \notin G$. Then

$$
\begin{aligned}
& { }^{G} r_{P}\left[\begin{array}{r}
r^{+} \\
r^{+}
\end{array}\right](x \cdot y)=r^{+} \text {or }{ }^{G} r_{P}\left[\begin{array}{l}
r_{r}^{-} \\
r^{+}
\end{array}\right](x)=r^{+}, \\
& { }^{G} g_{P}\left[\left[_{g^{+}}^{g^{-}}\right](x \cdot y)=g^{+} \text {or }{ }^{G} g_{P}\left[{ }_{g^{+}}^{g^{-}}\right](x)=g^{+},\right. \\
& { }^{G} b_{P}\left[b_{b^{-}}^{b^{+}}\right](x \cdot y)=b^{-} \text {or }{ }^{G} b_{P}\left[b_{b^{+}}^{b^{+}}\right](x)=b^{-} .
\end{aligned}
$$

Thus

$$
\begin{aligned}
& \max \left\{{ }^{G} r_{\mathrm{P}}\left[\begin{array}{l}
r_{r^{+}}^{-} \\
r^{-}
\end{array}\right](x \cdot y),{ }^{G} r_{P}\left[\begin{array}{l}
r_{r}^{-} \\
r^{+}
\end{array}\right](x)\right\}=r^{+}, \\
& \max \left\{{ }^{G} g_{P}\left[\begin{array}{l}
g^{-} \\
g^{+}
\end{array}\right](x \cdot y),{ }^{G} g_{P}\left[\begin{array}{l}
g^{-} \\
{ }^{+}
\end{array}\right](x)\right\}=g^{+}, \\
& \min \left\{{ }^{G} b_{P}\left[\begin{array}{l}
b^{+} \\
b^{-}
\end{array}\right](x \cdot y),{ }^{G} b_{P}\left[\begin{array}{l}
b^{+} \\
b^{-}
\end{array}\right](x)\right\}=b^{-} .
\end{aligned}
$$

Therefore,

$$
\begin{aligned}
& { }^{G} r_{P}\left[\begin{array}{r}
r_{r^{+}}^{-}
\end{array}\right](x) \leqslant r^{+}=\max \left\{{ }^{G} r_{P}\left[\begin{array}{r}
r^{+} \\
r^{+}
\end{array}\right](x \cdot y),{ }^{G} r_{P_{P}}\left[\begin{array}{r}
r^{+} \\
r^{+}
\end{array}\right](x)\right\}, \\
& { }^{G} g_{P}\left[{ }_{g^{+}}^{g^{-}}\right](x) \leqslant g^{+}=\max \left\{{ }^{G} g_{P}\left[{ }_{g^{+}}^{g^{-}}\right](x \cdot y),{ }^{G} g_{P}\left[{ }_{g^{+}}^{g^{-}}\right](x)\right\}, \\
& { }^{G} b_{P}\left[\begin{array}{c}
b^{+} \\
b^{-}
\end{array}\right](x) \geqslant b^{-}=\min \left\{{ }^{G} b_{P}\left[b^{b^{+}}\right](x \cdot y),{ }^{G} b_{P}\left[b_{b^{-}}^{b^{+}}\right](x)\right\} \text {. }
\end{aligned}
$$

Hence, $G \mathrm{P}_{\mathrm{r}^{+}, \mathrm{g}^{+}, \mathrm{b}^{-}}^{\mathrm{r}^{-}, \mathrm{g}^{-}, \mathrm{b}^{+}}$is a special picture fuzzy UP-filter of $\mathrm{X}$.

Theorem 3.60. The PFS ${ }^{G} P\left[\begin{array}{l}r^{-}, \mathrm{g}^{-}, \mathrm{b}^{+} \\ \mathrm{r}^{+}, \mathrm{g}^{+}, \mathrm{b}^{-}\end{array}\right]$in $\mathrm{X}$ is a special picture fuzzy implicative UP-filter of $\mathrm{X}$ if and only if a nonempty subset $\mathrm{G}$ of $\mathrm{X}$ is an implicative UP-filter of $\mathrm{X}$. 
Proof. Assume that $\mathrm{GP}_{\mathrm{r}^{+}, \mathrm{g}^{+}, \mathrm{b}^{-}}^{\mathrm{r}^{-}, \mathrm{g}^{-}, \mathrm{b}^{+}}$is a special picture fuzzy implicative UP-filter of $\mathrm{X}$. Since ${ }^{\mathrm{G}} \mathrm{P}_{\mathrm{r}^{+}, \mathrm{g}^{+}, \mathrm{b}^{-}}^{\mathrm{r}^{-}, \mathrm{g}^{-}, \mathrm{b}^{+}}$ satisfies the condition (3.7), it follows from Lemma 3.56 that $0 \in G$. Next, let $x, y, z \in X$ be such that $x \cdot(y \cdot z) \in G$ and $x \cdot y \in G$. Then ${ }^{G} r_{\mathrm{P}}\left[\begin{array}{c}r^{-} \\ r^{-}\end{array}\right](x \cdot(y \cdot z))=r^{-}={ }^{G} r_{P_{P}}\left[\begin{array}{r}r^{-} \\ r^{+}\end{array}\right](x \cdot y)$. Thus

$$
\left.{ }^{G} r_{P}\left[r_{r^{+}}^{r^{-}}\right](x \cdot z) \leqslant \max \left\{{ }^{G} r_{P}\left[r_{r^{+}}^{r^{-}}\right](x \cdot(y \cdot z)),{ }^{G} r_{P}\left[r_{r^{+}}^{r^{-}}\right](x \cdot y)\right\}=\max \left\{r^{-}, r^{-}\right\}=r^{-} \leqslant{ }^{G} r_{P_{P}}{ }_{r^{+}}^{r^{-}}\right](x \cdot z) \text { by (3.13) }
$$

and so ${ }^{G} r_{P}\left[\begin{array}{r}r^{-} \\ r^{-}\end{array}\right](x \cdot z)=r^{-}$. Thus $x \cdot z \in G$. Hence, $G$ is an implicative UP-filter of $X$.

Conversely, assume that $G$ is an implicative UP-filter of $X$. Since $0 \in G$, it follows from Lemma 3.55 that ${ }^{G} P\left[\begin{array}{l}r^{-}, g^{-}, b^{+} \\ r^{+}, \mathfrak{b}^{+}\end{array}\right]$satisfies the conditions (3.7), (3.8), and (3.9). Next, let $x, y, z \in X$.

Case 1: $x \cdot(y \cdot z) \in G$ and $x \cdot y \in G$. Then

$$
\begin{aligned}
& { }^{G} r_{P}\left[\begin{array}{l}
r_{r^{+}}^{-} \\
r^{-}
\end{array}\right](x \cdot(y \cdot z))=r^{-}={ }^{G} r_{P}\left[\begin{array}{l}
r_{r^{+}}^{-} \\
r^{+}
\end{array}(x \cdot y),\right. \\
& { }^{G} g_{P}\left[\begin{array}{l}
g^{+} \\
g^{+}
\end{array}\right](x \cdot(y \cdot z))=g^{-}={ }^{G} g_{P}\left[\begin{array}{l}
g^{-} \\
g^{+}
\end{array}\right](x \cdot y), \\
& { }^{G} b_{P}\left[\begin{array}{l}
b^{+} \\
b^{-}
\end{array}\right](x \cdot(y \cdot z))=b^{+}={ }^{G} b_{P}\left[b_{b^{-}}^{b^{+}}\right](x \cdot y) .
\end{aligned}
$$

Thus

$$
\begin{aligned}
& \max \left\{{ }^{G} r_{P}\left[\begin{array}{l}
{ }_{r^{+}}^{r^{-}}
\end{array}\right](x \cdot(y \cdot z)),{ }^{G} r_{P}\left[{ }_{r^{+}}^{r^{-}}\right](x \cdot y)\right\}=\max \left\{r^{-}, r^{-}\right\}=r^{-}, \\
& \max \left\{{ }^{G} g_{P}\left[{ }_{g^{+}}^{g^{-}}\right](x \cdot(y \cdot z)),{ }^{G} g_{P}\left[{ }_{g^{+}}^{g^{-}}\right](x \cdot y)\right\}=\max \left\{g^{-}, g^{-}\right\}=g^{-}, \\
& \min \left\{{ }^{G} b_{P}\left[{ }_{b^{-}}^{b^{+}}\right](x \cdot(y \cdot z)),{ }^{G} b_{P}\left[{ }_{b^{-}}^{b^{+}}\right](x \cdot y)\right\}=\min \left\{b^{+}, b^{+}\right\}=b^{+} .
\end{aligned}
$$

Since $G$ is an implicative UP-filter of $X$, we have $x \cdot z \in G$ and so ${ }^{G} r_{P}\left[\begin{array}{r}r^{+} \\ r^{+}\end{array}\right](x \cdot z)=r^{-},{ }^{G} g_{P}\left[\begin{array}{l}g^{-} \\ g^{+}\end{array}\right](x \cdot z)=g^{-}$, and ${ }^{G} b_{P}\left[\begin{array}{l}b^{+} \\ b^{-}\end{array}\right](x \cdot z)=b^{+}$. Thus

$$
\begin{aligned}
& { }^{G} r_{P}\left[\begin{array}{r}
r^{-} \\
r^{+}
\end{array}\right](x \cdot z)=r^{-} \leqslant r^{-}=\max \left\{{ }^{G} r_{P}\left[\begin{array}{r}
r^{-} \\
r^{-}
\end{array}\right](x \cdot(y \cdot z)),{ }^{G} r_{P}\left[\begin{array}{r}
r^{+} \\
r^{-}
\end{array}\right](x \cdot y)\right\}, \\
& { }^{G} g_{P}\left[g_{g^{+}}^{g^{-}}\right](x \cdot z)=g^{-} \leqslant g^{-}=\max \left\{{ }^{G} g_{P}\left[{ }_{g^{+}}^{g^{-}}\right](x \cdot(y \cdot z)),{ }^{G} g_{P}\left[g_{g^{+}}^{g^{-}}\right](x \cdot y)\right\} \text {, } \\
& { }^{\mathrm{G}} \mathrm{b}_{\mathrm{P}}\left[{ }_{\mathrm{b}^{-}}^{\mathrm{b}^{+}}\right](x \cdot z)=\mathrm{b}^{+} \geqslant \mathrm{b}^{+}=\min \left\{{ }^{\mathrm{G}} \mathrm{b}_{\mathrm{P}}\left[\mathrm{b}^{-}\right](x \cdot(y \cdot z)),{ }^{\mathrm{G}^{+}} \mathrm{b}_{\mathrm{P}}\left[{ }_{\mathrm{b}^{-}}^{\mathrm{b}^{+}}\right](x \cdot y)\right\} \text {. }
\end{aligned}
$$

Case 2: $x \cdot(y \cdot z) \notin G$ or $x \cdot y \notin G$. Then

$$
\begin{aligned}
& { }^{G} r_{P}\left[\begin{array}{l}
r_{r}^{-} \\
r^{+}
\end{array}\right](x \cdot(y \cdot z))=r^{+} \text {or }{ }^{G} r_{P}\left[\begin{array}{l}
r_{r}^{-} \\
r^{+}
\end{array}\right](x \cdot y)=r^{+}, \\
& { }^{G} g_{P}\left[\begin{array}{l}
g^{+} \\
g^{+}
\end{array}\right](x \cdot(y \cdot z))=g^{+} \text {or }{ }^{G} g_{P}\left[\begin{array}{l}
g^{+} \\
g^{+}
\end{array}\right](x \cdot y)=g^{+}, \\
& { }^{G} b_{P}\left[\begin{array}{l}
b^{+} \\
b^{-}
\end{array}\right](x \cdot(y \cdot z))=b^{-} \text {or }{ }^{G} b_{P}\left[\begin{array}{l}
b^{+} \\
b^{+}
\end{array}\right](x \cdot y)=b^{-} .
\end{aligned}
$$

Thus

$$
\begin{aligned}
& \max \left\{{ }^{G} r_{\mathrm{P}}\left[{ }_{r^{+}}^{r^{-}}\right](x \cdot(y \cdot z)),{ }^{G} r_{\mathrm{P}}\left[{ }_{r^{+}}^{r^{-}}\right](x \cdot y)\right\}=r^{+}, \\
& \max \left\{{ }^{G} g_{P}\left[{ }_{g^{+}}^{g^{-}}\right](x \cdot(y \cdot z)),{ }^{G} g_{P}\left[g_{g^{+}}^{g^{-}}\right](x \cdot y)\right\}=g^{+} \text {, } \\
& \min \left\{{ }^{\mathrm{G}} \mathrm{b}_{\mathrm{P}}\left[\mathrm{b}^{\mathrm{b}^{+}}\right](\mathrm{x} \cdot(\mathrm{y} \cdot z)),{ }^{\mathrm{G}} \mathrm{b}_{\mathrm{P}}\left[\mathrm{b}^{\mathrm{b}^{+}}\right](\mathrm{x} \cdot \mathrm{y})\right\}=\mathrm{b}^{-} \text {. }
\end{aligned}
$$

Therefore,

$$
\begin{aligned}
& { }^{\mathrm{G}} \mathrm{r}_{\mathrm{P}}\left[{ }_{\mathrm{r}^{+}}^{\mathrm{r}^{-}}\right](x \cdot z) \leqslant \mathrm{r}^{+}=\max \left\{{ }^{\mathrm{G}} \mathrm{r}_{\mathrm{P}}\left[{ }_{\mathrm{r}^{+}}^{\mathrm{r}^{-}}\right](x \cdot(y \cdot z)),{ }^{\mathrm{G}} \mathrm{r}_{\mathrm{P}}\left[{ }_{\mathrm{r}^{+}}^{\mathrm{r}^{-}}\right](x \cdot y)\right\}, \\
& { }^{G} g_{P}\left[g_{g^{+}}^{g^{-}}\right](x \cdot z) \leqslant g^{+}=\max \left\{{ }^{G} g_{P}\left[{ }_{g^{+}}^{g^{-}}\right](x \cdot(y \cdot z)),{ }^{G} g_{P}\left[g_{g^{+}}^{g^{-}}\right](x \cdot y)\right\}, \\
& { }^{G} b_{P}\left[b_{b^{-}}^{b^{+}}\right](x \cdot z) \geqslant b^{-}=\min \left\{{ }^{G} b_{P}\left[b^{b^{+}}\right](x \cdot(y \cdot z)),{ }^{G} b_{P}\left[b^{b^{+}}\right](x \cdot y)\right\} \text {. }
\end{aligned}
$$

Hence, ${ }^{G} \mathrm{P}_{\mathrm{r}^{+}, \mathrm{g}^{+}, \mathrm{b}^{-}}^{\mathrm{r}^{-}, \mathrm{g}^{-}, \mathrm{b}^{+}}$is a special picture fuzzy implicative UP-filter of $\mathrm{X}$. 


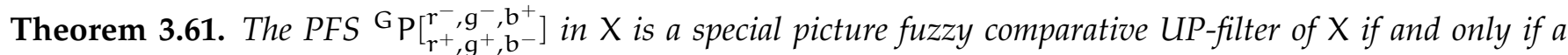
nonempty subset $\mathrm{G}$ of $\mathrm{X}$ is a comparative UP-filter of $\mathrm{X}$.

Proof. Assume that ${ }^{G}\left[\begin{array}{c}r^{-}, g^{-}, b^{+} \\ r^{+}, g^{+}, b^{-}\end{array}\right]$is a special picture fuzzy comparative UP-filter of $X$. Since $\left.{ }^{G} \mathrm{P}_{r^{+}}^{r^{-}, g^{+},,^{+}, b^{+}}\right]$ satisfies the condition (3.7), it follows from Lemma 3.56 that $0 \in G$. Next, let $x, y, z \in X$ be such that $x \cdot((y \cdot z) \cdot y) \in G$ and $x \in G$. Then ${ }^{G} r_{P_{P}}\left[\begin{array}{r}r^{-} \\ r^{+}\end{array}\right](x \cdot((y \cdot z) \cdot y))=r^{-}={ }^{G} r_{P}\left[\begin{array}{l}r^{+} \\ r^{-}\end{array}\right](x)$. Thus

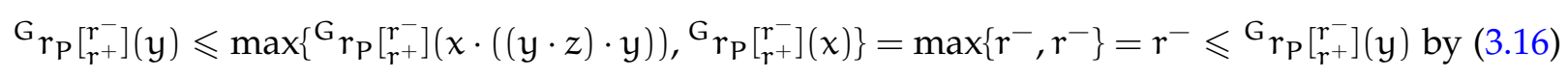

and so $\left.{ }^{G} r_{P}{ }_{r^{+}}^{r^{-}}\right](y)=r^{-}$. Thus $y \in G$. Hence, $G$ is a comparative UP-filter of $X$.

Conversely, assume that $G$ is a comparative UP-filter of $X$. Since $0 \in G$, it follows from Lemma 3.55 that ${ }^{G} P\left[\begin{array}{l}r^{-}, g^{-}, b^{+} \\ r^{+}, g^{+}, b^{-}\end{array}\right]$satisfies the conditions (3.7), (3.8), and (3.9). Next, let $x, y, z \in X$.

Case 1: $x \cdot((y \cdot z) \cdot y) \in G$ and $x \in G$. Then

$$
\begin{aligned}
& { }^{G} r_{P}\left[r_{r^{+}}^{r^{-}}\right](x \cdot((y \cdot z) \cdot y))=r^{-}={ }^{G} r_{P}\left[\begin{array}{l}
r_{r^{+}}^{-} \\
r^{-}
\end{array}(x),\right. \\
& { }^{G} g_{P}\left[{ }_{g^{+}}^{g^{-}}\right](x \cdot((y \cdot z) \cdot y))=g^{-}={ }^{G} g_{P}\left[g_{g^{+}}^{g^{-}}\right](x), \\
& { }^{G} b_{P}\left[b^{b^{+}}\right](x \cdot((y \cdot z) \cdot y))=b^{+}={ }^{G} b_{P}\left[{ }_{b^{-}}^{b^{+}}\right](x) \text {. }
\end{aligned}
$$

Thus

$$
\begin{gathered}
\max \left\{{ }^{G}{ }_{r_{P}}\left[r_{r^{+}}^{r^{-}}\right](x \cdot((y \cdot z) \cdot y)),{ }^{G} r_{P}\left[{ }_{r^{+}}^{r^{-}}\right](x)\right\}=\max \left\{r^{-}, r^{-}\right\}=r^{-}, \\
\max \left\{{ }^{G} g_{P}\left[{ }_{g^{+}}^{g^{-}}\right](x \cdot((y \cdot z) \cdot y)),{ }^{G} g_{P}\left[{ }_{g^{+}}^{g^{-}}\right](x)\right\}=\max \left\{g^{-}, g^{-}\right\}=g^{-}, \\
\min \left\{{ }^{G} b_{P}\left[b_{b^{+}}^{b^{+}}\right](x \cdot((y \cdot z) \cdot y)),{ }^{G} b_{P}\left[b_{b^{+}}^{b^{+}}\right](x)\right\}=\min \left\{b^{+}, b^{+}\right\}=b^{+} .
\end{gathered}
$$

Since $G$ is a comparative UP-filter of $X$, we have $y \in G$ and so ${ }^{G} r_{P}\left[r_{r^{+}}^{r^{-}}\right](y)=r^{-}, G g_{P}\left[g_{g^{+}}^{g^{-}}\right](y)=g^{-}$, and ${ }^{G} b_{\mathrm{P}}\left[b_{b^{-}}^{b^{+}}\right](y)=b^{+}$. Thus

$$
\begin{aligned}
& { }^{G} r_{P}\left[\begin{array}{r}
r_{r^{-}}^{-}
\end{array}\right](y)=r^{-} \leqslant r^{-}=\max \left\{{ }^{G} r_{P}\left[\begin{array}{l}
r_{r^{+}}^{-}
\end{array}\right](x \cdot((y \cdot z) \cdot y)),{ }^{G} r_{P}\left[{ }_{r^{+}}^{r^{-}}\right](x)\right\}, \\
& { }^{G} g_{P}\left[\begin{array}{l}
g^{+} \\
g^{+}
\end{array}\right](y)=g^{-} \leqslant g^{-}=\max \left\{{ }^{G} g_{P}\left[g_{g^{+}}^{g^{-}}\right](x \cdot((y \cdot z) \cdot y)),{ }^{G} g_{P}\left[\begin{array}{c}
g^{+} \\
g^{-}
\end{array}\right](x)\right\}, \\
& { }^{G} b_{P}\left[\begin{array}{l}
b^{+} \\
b^{-}
\end{array}\right](y)=b^{+} \geqslant b^{+}=\min \left\{{ }^{G} b_{P}\left[\begin{array}{l}
b^{+} \\
b^{-}
\end{array}\right](x \cdot((y \cdot z) \cdot y)),{ }^{G} b_{P}\left[\begin{array}{l}
b^{+} \\
b^{-}
\end{array}\right](x)\right\} \text {. }
\end{aligned}
$$

Case 2: $x \cdot((y \cdot z) \cdot y) \notin \mathrm{G}$ or $x \notin \mathrm{G}$. Then

$$
\begin{aligned}
& { }^{G} r_{P}\left[\begin{array}{r}
r^{+} \\
r^{+}
\end{array}\right](x \cdot((y \cdot z) \cdot y))=r^{+} \text {or }{ }^{G} r_{P}\left[\begin{array}{r}
r^{+} \\
r^{-}
\end{array}\right](x)=r^{+}, \\
& { }^{G} g_{P}\left[\begin{array}{l}
g^{-} \\
g^{+}
\end{array}\right](x \cdot((y \cdot z) \cdot y))=g^{+} \text {or }{ }^{G} g_{P}\left[\begin{array}{l}
g^{-} \\
g^{+}
\end{array}\right](x)=g^{+}, \\
& { }^{G} b_{P}\left[\begin{array}{l}
b^{+} \\
b^{-}
\end{array}\right](x \cdot((y \cdot z) \cdot y))=b^{-} \text {or }{ }^{G} b_{P}\left[\begin{array}{l}
b^{+} \\
b^{-}
\end{array}\right](x)=b^{-} .
\end{aligned}
$$

Thus

$$
\begin{aligned}
& \max \left\{{ }^{G} r_{\mathrm{P}}\left[r_{r^{+}}^{r^{-}}\right](x \cdot((y \cdot z) \cdot y)),{ }^{G} r_{P}\left[r_{r^{+}}^{r^{-}}\right](x)\right\}=r^{+}, \\
& \max \left\{{ }^{G} g_{P}\left[{ }_{\mathbf{g}^{+}}^{g^{-}}\right](x \cdot((y \cdot z) \cdot y)),{ }^{G} g_{P}\left[{ }_{\mathbf{g}^{+}}^{g^{-}}\right](x)\right\}=g^{+} \text {, } \\
& \min \left\{{ }^{G} b_{P}\left[{ }_{b^{-}}^{b^{+}}\right](x \cdot((y \cdot z) \cdot y)),{ }^{G} b_{P}\left[b^{b^{+}}\right](x)\right\}=b^{-} \text {. }
\end{aligned}
$$

Therefore,

$$
\begin{aligned}
& { }^{G} r_{P}\left[\begin{array}{r}
r_{r^{+}}^{-}
\end{array}\right](y) \leqslant r^{+}=\max \left\{{ }^{G} r_{P}\left[\begin{array}{r}
r_{r^{+}}^{-} \\
r^{-}
\end{array}\right](x \cdot((y \cdot z) \cdot y)),{ }^{G} r_{P}\left[\begin{array}{r}
r^{-} \\
r^{-}
\end{array}\right](x)\right\}, \\
& { }^{G} g_{P}\left[g_{g^{+}}^{g^{-}}\right](y) \leqslant g^{+}=\max \left\{{ }^{G} g_{P}\left[g^{+}\right](x \cdot((y \cdot z) \cdot y)),{ }^{g^{-}} g_{P}\left[g_{g^{+}}^{g^{-}}\right](x)\right\}, \\
& { }^{G} b_{P}\left[{ }_{b^{-}}^{b^{+}}\right](y) \geqslant b^{-}=\min \left\{{ }^{G} b_{P}\left[b_{b^{-}}^{b^{+}}\right](x \cdot((y \cdot z) \cdot y)),{ }^{G} b_{P}\left[\begin{array}{l}
b^{+} \\
b^{-}
\end{array}\right](x)\right\} \text {. }
\end{aligned}
$$

Hence, ${ }^{G} P\left[\begin{array}{c}r^{-}, \\ r^{+}, g^{+}, b^{-}\end{array}\right]$is a special picture fuzzy comparative UP-filter of $X$. 
Theorem 3.62. The PFS G $\mathrm{P}_{\mathrm{r}^{+}}^{\mathrm{r}^{-}, \mathrm{g}^{+}, \mathrm{b}^{+}}$, in $\mathrm{X}$ is a special picture fuzzy shift UP-filter of $\mathrm{X}$ if and only if a nonempty subset $\mathrm{G}$ of $\mathrm{X}$ is a shift UP-filter of $\mathrm{X}$.

Proof. Assume that $\left.{ }^{G} \mathrm{P}_{r^{+}}^{r^{-}, g^{+}, b^{-}}, b^{+}\right]$is a special picture fuzzy shift UP-filter of X. Since $\left.{ }^{G} \mathrm{P}_{r^{+}}^{r^{-}, g^{+}, b^{-}}, b^{+}\right]$satisfies the condition (3.7), it follows from Lemma 3.56 that $0 \in \mathrm{G}$. Next, let $x, y, z \in X$ be such that $x \cdot(y \cdot z) \in \mathrm{G}$ and $x \in G$. Then ${ }^{G} r_{P}\left[r_{r^{+}}^{r^{-}}\right](x \cdot(y \cdot z))=r^{-}={ }^{G} r_{P}\left[{ }_{r^{+}}^{r^{-}}\right](x)$. Thus

$$
\begin{aligned}
\left.{ }^{G}{ }_{r_{P}} r_{r^{+}}^{r^{-}}\right](((z \cdot y) \cdot y) \cdot z) & \leqslant \max \left\{{ }^{G}{ }_{r_{P}}\left[{ }_{r^{+}}^{r^{-}}\right](x \cdot(y \cdot z)),{ }^{G}{ }_{r_{P}}\left[{ }_{r^{+}}^{r^{-}}\right](x)\right\} \\
& =\max \left\{r^{-}, r^{-}\right\}=r^{-} \leqslant{ }^{G}{ }_{r_{P}}\left[{ }_{r^{+}}^{r^{-}}\right](((z \cdot y) \cdot y) \cdot z) \text { by (3.19) }
\end{aligned}
$$

and so ${ }^{G} r_{P}\left[{ }_{r^{+}}^{r^{-}}\right](((z \cdot y) \cdot y) \cdot z)=r^{-}$. Thus $((z \cdot y) \cdot y) \cdot z \in G$. Hence, $G$ is a shift UP-filter of $X$.

Conversely, assume that $G$ is a shift UP-filter of $X$. Since $0 \in G$, it follows from Lemma 3.55 that ${ }^{G} P\left[\begin{array}{c}r^{+} \\ r^{+}, g^{+}, b^{-}\end{array}\right]$satisfies the conditions (3.7), (3.8), and (3.9). Next, let $x, y, z \in X$.

Case 1: $x \cdot(y \cdot z) \in G$ and $x \in G$. Then

$$
\begin{aligned}
& { }^{G} r_{P}\left[\begin{array}{l}
r^{-} \\
r^{+}
\end{array}\right](x \cdot(y \cdot z))=r^{-}={ }^{G} r_{P}\left[\begin{array}{l}
r_{r}^{+} \\
r^{+}
\end{array}\right](x), \\
& { }^{G} g_{P}\left[\begin{array}{l}
g^{-} \\
{ }^{+}
\end{array}\right](x \cdot(y \cdot z))=g^{-}={ }^{G} g_{P}\left[\begin{array}{l}
g^{-} \\
g^{+}
\end{array}\right](x), \\
& { }^{G} b_{P}\left[\begin{array}{l}
b^{+} \\
{ }^{-}
\end{array}\right](x \cdot(y \cdot z))=b^{+}={ }^{G} b_{P}\left[\begin{array}{l}
b^{+} \\
b^{-}
\end{array}\right](x) .
\end{aligned}
$$

Thus

$$
\begin{aligned}
& \left.\max \left\{{ }^{\mathrm{G}} \mathrm{r}_{\mathrm{P}}\left[{ }_{\mathbf{r}^{+}}^{\mathrm{r}^{-}}\right](\mathrm{x} \cdot(\mathrm{y} \cdot z)),{ }^{\mathrm{G}} \mathrm{r}_{\mathrm{P}}{ }_{\mathrm{r}^{+}}^{\mathrm{r}^{-}}\right](\mathrm{x})\right\}=\max \left\{\mathrm{r}^{-}, \mathrm{r}^{-}\right\}=\mathrm{r}^{-}, \\
& \max \left\{{ }^{G} g_{P}\left[g_{g^{+}}^{g^{-}}\right](x \cdot(y \cdot z)),{ }^{G} g_{P}\left[g_{g^{+}}^{g^{-}}\right](x)\right\}=\max \left\{g^{-}, g^{-}\right\}=g^{-} \text {, } \\
& \min \left\{{ }^{G} b_{P}\left[\begin{array}{l}
b^{+} \\
b^{-}
\end{array}\right](x \cdot(y \cdot z)),{ }^{G} b_{P}\left[b^{b^{+}}\right](x)\right\}=\min \left\{b^{+}, b^{+}\right\}=b^{+} \text {. }
\end{aligned}
$$

Since $G$ is a shift UP-filter of $X$, we have $((z \cdot y) \cdot y) \cdot z \in G$ and so ${ }^{G} r_{P}\left[\begin{array}{r}r^{-} \\ r^{-}\end{array}\right](((z \cdot y) \cdot y) \cdot z)=r^{-},{ }^{G} g_{P}\left[{ }_{g^{+}}^{g^{-}}\right](((z$. $y) \cdot y) \cdot z)=g^{-}$, and ${ }^{G} b_{P}\left[b_{b^{-}}^{b^{+}}\right](((z \cdot y) \cdot y) \cdot z)=b^{+}$. Thus

$$
\begin{aligned}
& { }^{\mathrm{G}} \mathrm{r}_{\mathrm{P}}\left[{ }_{\mathrm{r}^{+}}^{\mathrm{r}^{-}}\right](((z \cdot y) \cdot y) \cdot z)=\mathrm{r}^{-} \leqslant \mathrm{r}^{-}=\max \left\{{ }^{\mathrm{G}} \mathrm{r}_{\mathrm{P}}\left[\begin{array}{r}
\mathrm{r}^{+} \\
\mathrm{r}^{-}
\end{array}\right](x \cdot(y \cdot z)),{ }^{\mathrm{G}} \mathrm{r}_{\mathrm{P}}\left[{ }_{\mathrm{r}^{+}}^{\mathrm{r}^{-}}\right](x)\right\}, \\
& { }^{G} g_{P}\left[g_{g^{+}}^{g^{-}}\right](((z \cdot y) \cdot y) \cdot z)=g^{-} \leqslant g^{-}=\max \left\{{ }^{G} g_{P}\left[g_{g^{+}}^{g^{-}}\right](x \cdot(y \cdot z)),{ }^{G} g_{P}\left[{ }_{g^{+}}^{g^{-}}\right](x)\right\}, \\
& { }^{G} b_{P}\left[b_{b^{-}}^{b^{+}}\right](((z \cdot y) \cdot y) \cdot z)=b^{+} \geqslant b^{+}=\min \left\{{ }^{G} b_{P}\left[b^{b^{+}}\right](x \cdot(y \cdot z)),{ }^{G} b_{P}\left[b_{b^{-}}^{b^{+}}\right](x)\right\} \text {. }
\end{aligned}
$$

Case 2: $x \cdot(y \cdot z) \notin \mathrm{G}$ or $x \notin \mathrm{G}$. Then

$$
\begin{aligned}
& { }^{G} r_{P}\left[\begin{array}{l}
r_{r^{-}}^{-}
\end{array}\right](x \cdot(y \cdot z))=r^{+} \text {or }{ }^{G} r_{P}\left[\begin{array}{r}
r_{r+}^{-} \\
r^{+}
\end{array}\right](x)=r^{+}, \\
& { }^{G} g_{P}\left[g_{g^{+}}^{g^{-}}\right](x \cdot(y \cdot z))=g^{+} \text {or }{ }^{G} g_{P}\left[g_{g^{+}}^{g^{-}}\right](x)=g^{+} \text {, } \\
& { }^{G} b_{P}\left[\begin{array}{c}
b^{+} \\
b^{-}
\end{array}\right](x \cdot(y \cdot z))=b^{-} \text {or }{ }^{G} b_{P}\left[\begin{array}{l}
b^{+} \\
b^{-}
\end{array}\right](x)=b^{-} \text {. }
\end{aligned}
$$

Thus

$$
\begin{aligned}
& \max \left\{{ }^{G} r_{\mathrm{P}}\left[r_{r^{+}}^{r^{-}}\right](x \cdot(y \cdot z)),{ }^{G} r_{\mathrm{P}}\left[r_{r^{+}}^{r^{-}}\right](x)\right\}=r^{+}, \\
& \max \left\{{ }^{G} g_{P}\left[{ }_{g^{+}}^{g^{-}}\right](x \cdot(y \cdot z)),{ }^{G} g_{P}\left[{ }_{g^{+}}^{g^{-}}\right](x)\right\}=g^{+}, \\
& \min \left\{{ }^{\mathrm{G}} \mathrm{b}_{\mathrm{P}}\left[\mathrm{b}_{\mathrm{b}^{-}}^{\mathrm{b}^{+}}\right](\mathrm{x} \cdot(\mathrm{y} \cdot z)),{ }^{\mathrm{G}} \mathrm{b}_{\mathrm{P}}\left[{ }_{\mathrm{b}^{-}}^{\mathrm{b}^{+}}\right](\mathrm{x})\right\}=\mathrm{b}^{-} .
\end{aligned}
$$

Therefore,

$$
{ }^{G} r_{P}\left[{ }_{r^{+}}^{r^{-}}\right](((z \cdot y) \cdot y) \cdot z) \leqslant r^{+}=\max \left\{{ }^{G} r_{P}\left[r_{r^{+}}^{r^{-}}\right](x \cdot(y \cdot z)),{ }^{G} r_{P}\left[\begin{array}{r}
r^{+} \\
r^{-}
\end{array}\right](x)\right\}
$$




$$
\begin{aligned}
& { }^{G} g_{P}\left[{ }_{g^{+}}^{g^{-}}\right](((z \cdot y) \cdot y) \cdot z) \leqslant g^{+}=\max \left\{{ }^{G} g_{P}\left[\begin{array}{l}
g^{+} \\
g^{+}
\end{array}\right](x \cdot(y \cdot z)),{ }^{G} g_{P}\left[\begin{array}{l}
g^{+} \\
g^{-}
\end{array}\right](x)\right\}, \\
& { }^{G} b_{P}\left[b_{b^{+}}^{b^{+}}\right](((z \cdot y) \cdot y) \cdot z) \geqslant b^{-}=\min \left\{{ }^{G} b_{P}\left[b_{b^{-}}^{b^{+}}\right](x \cdot(y \cdot z)),{ }^{G} b_{P}\left[b^{b}\right](x)\right\} .
\end{aligned}
$$

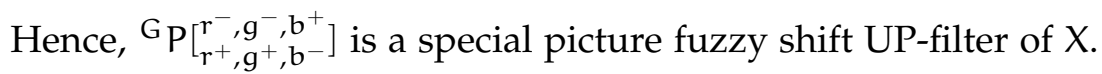

Theorem 3.63. The PFS ${ }^{G} \mathrm{P}_{\mathbf{r}^{+}}^{r^{-}, \mathbf{g}^{+}, \mathrm{b}^{-}}, \mathrm{b}^{+}$in $\mathrm{X}$ is a special picture fuzzy UP-ideal of $\mathrm{X}$ if and only if a nonempty subset $\mathrm{G}$ of $\mathrm{X}$ is a UP-ideal of $\mathrm{X}$.

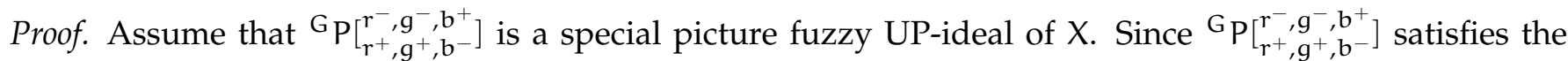
condition (3.7), it follows from Lemma 3.56 that $0 \in \mathrm{G}$. Next, let $x, y, z \in X$ be such that $x \cdot(y \cdot z) \in G$ and $y \in G$. Then ${ }^{G} r_{P_{P}}\left[\begin{array}{l}r^{-} \\ r^{+}\end{array}\right](x \cdot(y \cdot z))=r^{-}={ }^{G} r_{P}\left[\begin{array}{l}r^{+} \\ r^{-}\end{array}\right](y)$. Thus

$$
{ }^{G} r_{P}\left[\begin{array}{r}
r^{+} \\
r^{-}
\end{array}\right](x \cdot z) \leqslant \max \left\{{ }^{G} r_{P}\left[\begin{array}{r}
r_{r^{+}}^{-}
\end{array}\right](x \cdot(y \cdot z)),{ }^{G} r_{P}\left[\begin{array}{r}
r^{-} \\
r^{-}
\end{array}\right](y)\right\}=\max \left\{r^{-}, r^{-}\right\}=r^{-} \leqslant{ }^{G} r_{P}\left[\begin{array}{r}
r_{r}^{-} \\
r^{-}
\end{array}\right](x \cdot z) \text { by }(3.22)
$$

and so ${ }^{G} \mathrm{r}_{\mathrm{P}}\left[{ }_{\mathrm{r}^{+}}^{r^{-}}\right](x \cdot z)=\mathrm{r}^{-}$. Thus $x \cdot z \in \mathrm{G}$. Hence, $\mathrm{G}$ is a UP-ideal of $X$.

Conversely, assume that $G$ is a UP-ideal of $X$. Since $0 \in G$, it follows from Lemma 3.55 that $G P\left[\begin{array}{l}r^{+}, g^{+}, b^{-} \\ r^{-}, b^{+}\end{array}\right]$ satisfies the conditions (3.7), (3.8), and (3.9). Next, let $x, y, z \in X$.

Case 1: $x \cdot(y \cdot z) \in G$ and $y \in G$. Then

$$
\begin{aligned}
& { }^{G} r_{P}\left[\begin{array}{l}
r_{r}^{-} \\
r^{+}
\end{array}\right](x \cdot(y \cdot z))=r^{-}={ }^{G} r_{P}\left[\begin{array}{l}
r_{r}^{-} \\
r^{+}
\end{array}\right](y), \\
& { }^{G} g_{P}\left[\begin{array}{l}
g^{-} \\
g^{+}
\end{array}\right](x \cdot(y \cdot z))=g^{-}={ }^{G} g_{P}\left[\begin{array}{l}
g^{-} \\
g^{+}
\end{array}\right](y), \\
& { }^{G} b_{P}\left[\begin{array}{l}
b^{+} \\
b^{-}
\end{array}\right](x \cdot(y \cdot z))=b^{+}={ }^{G} b_{P}\left[\begin{array}{l}
b^{+} \\
b^{-}
\end{array}\right](y) .
\end{aligned}
$$

Thus

$$
\begin{aligned}
& \max \left\{{ }^{G} r_{P}\left[\begin{array}{r}
r^{+} \\
r^{+}
\end{array}\right](x \cdot(y \cdot z)),{ }^{G} r_{P}\left[\begin{array}{c}
r^{+} \\
r^{-}
\end{array}\right](y)\right\}=\max \left\{r^{-}, r^{-}\right\}=r^{-}, \\
& \max \left\{{ }^{G} g_{P}\left[{ }_{g^{+}}^{g^{-}}\right](x \cdot(y \cdot z)),{ }^{G} g_{P}\left[\begin{array}{c}
g^{+} \\
g^{-}
\end{array}\right](y)\right\}=\max \left\{g^{-}, g^{-}\right\}=g^{-}, \\
& \min \left\{{ }^{G} b_{P}\left[b^{b^{+}}\right](x \cdot(y \cdot z)),{ }^{G} b_{P}\left[\begin{array}{l}
b^{+} \\
b^{-}
\end{array}\right](y)\right\}=\min \left\{b^{+}, b^{+}\right\}=b^{+} .
\end{aligned}
$$

Since $G$ is a UP-ideal of $X$, we have $x \cdot z \in G$ and so ${ }^{G} r_{P}\left[\begin{array}{r}r^{+} \\ r^{-}\end{array}\right](x \cdot z)=r^{-},{ }^{G} g_{P}\left[g^{g^{-}}\right](x \cdot z)=g^{-}$, and ${ }^{\mathrm{G}} \mathrm{b}_{\mathrm{P}}\left[\mathrm{b}^{+}\right](\mathrm{x} \cdot z)=\mathrm{b}^{+}$. Thus

$$
\begin{aligned}
& { }^{G} r_{P}\left[\begin{array}{r}
r^{+} \\
r^{+}
\end{array}\right](x \cdot z)=r^{-} \leqslant r^{-}=\max \left\{{ }^{G} r_{P}\left[\begin{array}{r}
r_{r}^{-} \\
r^{+}
\end{array}\right](x \cdot(y \cdot z)),{ }^{G} r_{P}\left[\begin{array}{r}
r_{r}^{-} \\
r^{-}
\end{array}\right](y)\right\}, \\
& { }^{G} g_{P}\left[g_{g^{+}}^{g^{-}}\right](x \cdot z)=g^{-} \leqslant g^{-}=\max \left\{{ }^{G} g_{P}\left[{ }_{g^{+}}^{g^{-}}\right](x \cdot(y \cdot z)),{ }^{G} b_{P}\left[g_{g^{+}}^{g^{-}}\right](y)\right\}, \\
& { }^{G} b_{P}\left[b_{b^{-}}^{b^{+}}\right](x \cdot z)=b^{+} \geqslant b^{+}=\min \left\{{ }^{G} b_{P}\left[b^{b^{+}}\right](x \cdot(y \cdot z)),{ }^{G} b_{P}\left[{ }_{b^{-}}^{b^{+}}\right](y)\right\} \text {. }
\end{aligned}
$$

Case 2: $x \cdot(y \cdot z) \notin G$ or $y \notin G$. Then

$$
\begin{aligned}
& { }^{{ }^{G}} r_{P}\left[\begin{array}{c}
r^{+} \\
r^{+}
\end{array}\right](x \cdot(y \cdot z))=r^{+} \text {or }{ }^{G} r_{P}\left[\begin{array}{r}
r^{+} \\
r^{+}
\end{array}\right](y)=r^{+}, \\
& { }^{G} g_{P}\left[\begin{array}{l}
g^{-} \\
g^{+}
\end{array}\right](x \cdot(y \cdot z))=g^{+} \text {or }{ }^{G} g_{P}\left[\begin{array}{l}
g^{-} \\
g^{+}
\end{array}\right](y)=g^{+}, \\
& { }^{G} b_{P}\left[\begin{array}{l}
b^{+} \\
b^{-}
\end{array}\right](x \cdot(y \cdot z))=b^{-} \text {or }{ }^{G} b_{P}\left[b_{b^{-}}^{b^{+}}\right](y)=b^{-} .
\end{aligned}
$$

Thus

$$
\begin{gathered}
\max \left\{{ }^{G} r_{\mathrm{P}}\left[{ }_{r^{+}}^{r^{-}}\right](x \cdot(y \cdot z)),{ }^{G} r_{P}\left[{ }_{r^{+}}^{r^{-}}\right](y)\right\}=r^{+}, \\
\max \left\{{ }^{G} g_{P}\left[{ }_{g^{+}}^{g^{-}}\right](x \cdot(y \cdot z)),{ }^{G} g_{P}\left[{ }_{g^{+}}^{g^{-}}\right](y)\right\}=g^{+},
\end{gathered}
$$




$$
\min \left\{{ }^{G} b_{P}\left[b_{b^{-}}^{b^{+}}\right](x \cdot(y \cdot z)),{ }^{G} b_{P}\left[b^{b^{+}}\right](y)\right\}=b^{-} .
$$

Therefore,

$$
\begin{aligned}
& { }^{G} r_{\mathrm{P}}\left[\begin{array}{c}
r_{r^{-}}^{+}
\end{array}\right](x \cdot z) \leqslant r^{+}=\max \left\{{ }^{G} r_{\mathrm{P}}\left[\begin{array}{c}
r_{r^{-}}^{+}
\end{array}\right](x \cdot(y \cdot z)),{ }^{G} r_{P}\left[\begin{array}{l}
r_{r^{+}}^{-}
\end{array}\right](y)\right\}, \\
& { }^{G} g_{P}\left[g_{g^{+}}^{g^{-}}\right](x \cdot z) \leqslant g^{+}=\max \left\{{ }^{G} g_{P}\left[{ }_{g^{+}}^{g^{-}}\right](x \cdot(y \cdot z)),{ }^{G} g_{P}\left[{ }_{g^{+}}^{g^{-}}\right](y)\right\}, \\
& { }^{G} b_{P}\left[{ }_{b^{-}}^{b^{+}}\right](x \cdot z) \geqslant b^{-}=\min \left\{{ }^{G} b_{P}\left[{ }_{b^{-}}^{b^{+}}\right](x \cdot(y \cdot z)),{ }^{G} b_{P}\left[{ }_{b^{-}}^{b^{+}}\right](y)\right\} \text {. }
\end{aligned}
$$

Hence, ${ }^{G} \mathrm{P}_{\mathrm{r}^{+}, \mathrm{g}^{+}, \mathrm{b}^{-}}^{\mathrm{r}^{-}, \mathrm{g}^{-}, \mathrm{b}^{+}}$is a special picture fuzzy UP-ideal of $\mathrm{X}$.

Theorem 3.64. The PFS ${ }^{G} \mathrm{P}_{\mathrm{r}^{+}}^{r^{-}, \mathrm{g}^{+}, \mathrm{b}^{+}}, \mathrm{b}^{+}$in $\mathrm{X}$ is a special picture fuzzy strong UP-ideal of $\mathrm{X}$ if and only if a nonempty subset $\mathrm{G}$ of $\mathrm{X}$ is a strong UP-ideal of $\mathrm{X}$.

Proof. Assume that $\mathrm{G}^{\mathrm{P}}\left[\begin{array}{c}\mathrm{r}^{-}, \mathrm{g}^{-}, \mathrm{b}^{+} \\ \mathrm{r}^{+}, \mathrm{b}^{-}\end{array}\right]$is a special picture fuzzy strong UP-ideal of X. By Theorem 3.19, we have ${ }^{G} P\left[\begin{array}{c}r_{r}^{-}, g^{-}, b^{+} \\ r^{+}, g^{+}, b^{-}\end{array}\right]$is constant, that is, ${ }^{G} r_{P}\left[\begin{array}{c}r_{r+}^{-} \\ r^{+}\end{array}\right]$is constant. Since $G$ is nonempty, we have ${ }^{G} r_{P}\left[\begin{array}{c}r^{+} \\ r^{-}\end{array}\right](x)=r^{-}$for all $x \in X$. Thus $G=X$. Hence, $G$ is a strong UP-ideal of $X$.

Conversely, assume that $\mathrm{G}$ is a strong UP-ideal of $X$. Then $G=X$, so

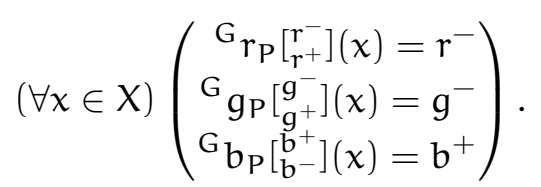

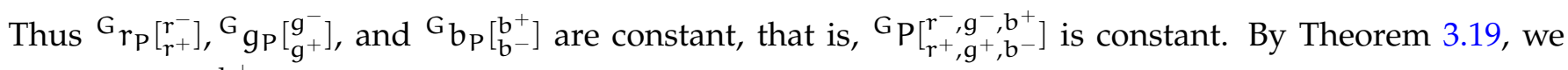
have ${ }^{G} P_{r^{+}, g^{+}, b^{-}}^{r^{-}}, g^{-}, b^{+}$is a special picture fuzzy strong UP-ideal of $X$.

\section{Level subsets of a PFS}

In this section, we discuss the relationships between special picture fuzzy UP-subalgebras (resp., special picture fuzzy near UP-filters, special picture fuzzy UP-filters, special picture fuzzy UP-ideals, special picture fuzzy strong UP-ideals) of UP-algebras and their level subsets.

Definition 4.1. [26] Let $f$ be a fuzzy set in $X$. For any $t \in[0,1]$, the sets

$$
\begin{array}{rlrl}
\mathrm{U}(f ; t) & =\{x \in X \mid f(x) \geqslant t\}, & \mathrm{U}^{+}(f ; t) & =\{x \in X \mid f(x)>t\}, \\
L(f ; t) & =\{x \in X \mid f(x) \leqslant t\}, & L^{-}(f ; t)=\{x \in X \mid f(x)<t\}, \\
E(f ; t) & =\{x \in X \mid f(x)=t\} &
\end{array}
$$

are called an upper $\mathrm{t}$-level subset, an upper $\mathrm{t}$-strong level subset, a lower $\mathrm{t}$-level subset, a lower $\mathrm{t}$-strong level subset and an equal $t$-level subset of $f$, respectively.

Theorem 4.2. A PFS $\mathrm{P}$ in $\mathrm{X}$ is a special picture fuzzy UP-subalgebra of $\mathrm{X}$ if and only if for all $\mathrm{t} \in[0,1]$, the sets $\mathrm{L}\left(\mathrm{r}_{\mathrm{P}} ; \mathrm{t}\right), \mathrm{L}\left(\mathrm{g}_{\mathrm{P}} ; \mathrm{t}\right)$, and $\mathrm{U}\left(\mathrm{b}_{\mathrm{P}} ; \mathrm{t}\right)$ are $\mathrm{UP}$-subalgebras of $\mathrm{X}$ if $\mathrm{L}\left(\mathrm{r}_{\mathrm{P}} ; \mathrm{t}\right), \mathrm{L}\left(\mathrm{g}_{\mathrm{P}} ; \mathrm{t}\right)$, and $\mathrm{U}\left(\mathrm{b}_{\mathrm{P}} ; \mathrm{t}\right)$ are nonempty.

Proof. Assume that $P$ is a special picture fuzzy UP-subalgebra of $X$. Let $t \in[0,1]$ be such that $L\left(r_{P} ; t\right)$, $\mathrm{L}\left(g_{P} ; t\right)$, and $\mathrm{U}\left(\mathrm{b}_{\mathrm{P}} ; \mathrm{t}\right)$ are nonempty.

Let $x, y \in L\left(r_{P} ; t\right)$. Then $r_{P}(x) \leqslant t$ and $r_{P}(y) \leqslant t$, so $t$ is an upper bound of $\left\{r_{P}(x), r_{P}(y)\right\}$. By (3.1), we have $r_{P}(x \cdot y) \leqslant \max \left\{r_{P}(x), r_{P}(y)\right\} \leqslant t$. Thus $x \cdot y \in L\left(r_{P} ; t\right)$.

Let $x, y \in L\left(g_{P} ; t\right)$. Then $g_{P}(x) \leqslant t$ and $g_{P}(y) \leqslant t$, so $t$ is an upper bound of $\left\{g_{P}(x), g_{P}(y)\right\}$. By (3.2), we have $g_{P}(x \cdot y) \leqslant \max \left\{g_{P}(x), g_{P}(y)\right\} \leqslant t$. Thus $x \cdot y \in L\left(g_{P} ; t\right)$.

Let $x, y \in U\left(b_{P} ; t\right)$. Then $b_{P}(x) \geqslant t$ and $b_{P}(y) \geqslant t$, so $t$ is a lower bound of $\left\{b_{P}(x), b_{P}(y)\right\}$. By (3.3), we have $b_{P}(x \cdot y) \geqslant \min \left\{b_{P}(x), b_{P}(y)\right\} \geqslant t$. Thus $x \cdot y \in U\left(b_{P} ; t\right)$. 
Hence, $\mathrm{L}\left(\mathrm{r}_{\mathrm{P}} ; \mathrm{t}\right), \mathrm{L}\left(\mathrm{g}_{\mathrm{P}} ; \mathrm{t}\right)$, and $\mathrm{U}\left(\mathrm{b}_{\mathrm{P}} ; \mathrm{t}\right)$ are UP-subalgebras of $\mathrm{X}$.

Conversely, assume that for all $t \in[0,1]$, the sets $L\left(r_{P} ; t\right), L\left(g_{P} ; t\right)$, and $U\left(b_{P} ; t\right)$ are UP-subalgebras of $\mathrm{L}\left(\mathrm{r}_{\mathrm{P}} ; \mathrm{t}\right), \mathrm{L}\left(\mathrm{g}_{\mathrm{P}} ; \mathrm{t}\right)$, and $\mathrm{U}\left(\mathrm{b}_{\mathrm{P}} ; \mathrm{t}\right)$ are nonempty.

Let $x, y \in X$. Then $r_{P}(x), r_{P}(y) \in[0,1]$. Choose $t=\max \left\{r_{P}(x), r_{P}(y)\right\}$. Thus $r_{P}(x) \leqslant t$ and $r_{P}(y) \leqslant t$, so $x, y \in L\left(r_{P} ; t\right) \neq \emptyset$. By assumption, we have $L\left(r_{P} ; t\right)$ is a UP-subalgebra of $X$ and so $x \cdot y \in L\left(r_{P} ; t\right)$. Thus $r_{P}(x \cdot y) \leqslant t=\max \left\{r_{P}(x), r_{P}(y)\right\}$.

Let $x, y \in X$. Then $g_{P}(x), g_{P}(y) \in[0,1]$. Choose $t=\max \left\{g_{P}(x), g_{P}(y)\right\}$. Thus $g_{P}(x) \leqslant t$ and $g_{P}(y) \leqslant t$, so $x, y \in \mathrm{L}\left(g_{P} ; t\right) \neq \emptyset$. By assumption, we have $\mathrm{L}\left(g_{P} ; t\right)$ is a UP-subalgebra of $X$ and so $x \cdot y \in L\left(g_{P} ; t\right)$. Thus $g_{P}(x \cdot y) \leqslant t=\max \left\{g_{P}(x), g_{P}(y)\right\}$.

Let $x, y \in X$. Then $b_{P}(x), b_{P}(y) \in[0,1]$. Choose $t=\min \left\{b_{P}(x), b_{P}(y)\right\}$. Thus $b_{P}(x) \geqslant t$ and $b_{P}(y) \geqslant t$, so $x, y \in U\left(b_{P} ; t\right) \neq \emptyset$. By assumption, we have $U\left(b_{P} ; t\right)$ is a UP-subalgebra of $X$ and so $x \cdot y \in U\left(b_{P} ; t\right)$. Thus $b_{P}(x \cdot y) \geqslant t=\min \left\{b_{P}(x), b_{P}(y)\right\}$.

Therefore, $\mathrm{P}$ is a special picture fuzzy UP-subalgebra of $\mathrm{X}$.

Theorem 4.3. If $\mathrm{P}$ is a special picture fuzzy UP-subalgebra of $\mathrm{X}$, then for all $\mathrm{t} \in[0,1]$, the sets $\mathrm{L}^{-}\left(\mathrm{r}_{\mathrm{P}} ; \mathrm{t}\right), \mathrm{L}^{-}\left(\mathrm{g}_{\mathrm{P}} ; \mathrm{t}\right)$, and $\mathrm{U}^{+}\left(\mathrm{b}_{\mathrm{P}} ; \mathrm{t}\right)$ are $\mathrm{UP}$-subalgebras of $\mathrm{X}$ if $\mathrm{L}^{-}\left(\mathrm{r}_{\mathrm{P}} ; \mathrm{t}\right), \mathrm{L}^{-}\left(\mathrm{g}_{\mathrm{P}} ; \mathrm{t}\right)$, and $\mathrm{U}^{+}\left(\mathrm{b}_{\mathrm{P}} ; \mathrm{t}\right)$ are nonempty.

Proof. Assume that $\mathrm{P}$ is a special picture fuzzy UP-subalgebra of $X$. Let $t \in[0,1]$ be such that $\mathrm{L}^{-}\left(\mathrm{r}_{\mathrm{P}} ; t\right)$, $\mathrm{L}^{-}\left(\mathrm{g}_{\mathrm{P}} ; \mathrm{t}\right)$, and $\mathrm{U}^{+}\left(\mathrm{b}_{\mathrm{P}} ; \mathrm{t}\right)$ are nonempty.

Let $x, y \in L^{-}\left(r_{P} ; t\right)$. Then $r_{P}(x)<t$ and $r_{P}(y)<t$, so $t$ is an upper bound of $\left\{r_{P}(x), r_{P}(y)\right\}$. By (3.1), we have $r_{P}(x \cdot y) \leqslant \max \left\{r_{P}(x), r_{P}(y)\right\}<t$. Thus $x \cdot y \in L^{-}\left(r_{P} ; t\right)$.

Let $x, y \in L^{-}\left(g_{P} ; t\right)$. Then $g_{P}(x)<t$ and $g_{P}(y)<t$, so $t$ is an upper bound of $\left\{g_{P}(x), g_{P}(y)\right\}$. By (3.2), we have $g_{P}(x \cdot y) \leqslant \max \left\{g_{P}(x), g_{P}(y)\right\}<t$. Thus $x \cdot y \in L^{-}\left(g_{P} ; t\right)$.

Let $x, y \in u^{+}\left(b_{P} ; t\right)$. Then $b_{P}(x)>t$ and $b_{P}(y)>t$, so $t$ is a lower bound of $\left\{b_{P}(x), b_{P}(y)\right\}$. By (3.3), we have $b_{P}(x \cdot y) \geqslant \min \left\{b_{P}(x), b_{P}(y)\right\}>t$. Thus $x \cdot y \in U^{+}\left(b_{P} ; t\right)$.

Hence, $\mathrm{L}^{-}\left(\mathrm{r}_{\mathrm{P}} ; \mathrm{t}\right), \mathrm{L}^{-}\left(g_{P} ; t\right)$, and $\mathrm{U}^{+}\left(b_{P} ; t\right)$ are UP-subalgebras of $X$.

Theorem 4.4. A PFS $\mathrm{P}$ in $\mathrm{X}$ is a special picture fuzzy near UP-filter of $\mathrm{X}$ if and only if for all $\mathrm{t} \in[0,1]$, the sets $\mathrm{L}\left(\mathrm{r}_{\mathrm{P}} ; \mathrm{t}\right), \mathrm{L}\left(\mathrm{g}_{\mathrm{P}} ; \mathrm{t}\right)$, and $\mathrm{U}\left(\mathrm{b}_{\mathrm{P}} ; \mathrm{t}\right)$ are near UP-filters of $\mathrm{X}$ if $\mathrm{L}\left(\mathrm{r}_{\mathrm{P}} ; \mathrm{t}\right), \mathrm{L}\left(\mathrm{g}_{\mathrm{P}} ; \mathrm{t}\right)$, and $\mathrm{U}\left(\mathrm{b}_{\mathrm{P}} ; \mathrm{t}\right)$ are nonempty.

Proof. Assume that $P$ is a special picture fuzzy near UP-filter of $X$. Let $t \in[0,1]$ be such that $L\left(r_{P} ; t\right)$, $\mathrm{L}\left(g_{P} ; t\right)$, and $\mathrm{U}\left(b_{P} ; t\right)$ are nonempty.

Let $x \in X$ and $y \in L\left(r_{P} ; t\right)$. Then $r_{P}(y) \leqslant t$. By (3.4), we have $r_{P}(x \cdot y) \leqslant r_{P}(y) \leqslant t$. Thus $x \cdot y \in L\left(r_{P} ; t\right)$. Let $x \in X$ and $y \in L\left(g_{P} ; t\right)$. Then $g_{P}(y) \leqslant t$. By (3.5), we have $g_{P}(x \cdot y) \leqslant g_{P}(y) \leqslant t$. Thus $x \cdot y \in L\left(g_{P} ; t\right)$. Let $x \in X$ and $y \in U\left(b_{P} ; t\right)$. Then $b_{P}(y) \geqslant t$. By (3.6), we have $b_{P}(x \cdot y) \geqslant b_{P}(y) \geqslant t$. Thus $x \cdot y \in U\left(b_{P} ; t\right)$.

Hence, $\mathrm{L}\left(\mathrm{r}_{\mathrm{P}} ; \mathrm{t}\right), \mathrm{L}\left(\mathrm{g}_{\mathrm{P}} ; \mathrm{t}\right)$, and $\mathrm{U}\left(\mathrm{b}_{\mathrm{P}} ; \mathrm{t}\right)$ are near UP-filters of $\mathrm{X}$.

Conversely, assume that for all $t \in[0,1]$, the sets $L\left(r_{P} ; t\right), L\left(g_{P} ; t\right)$, and $U\left(b_{P} ; t\right)$ are near UP-filters of $X$ if $L\left(r_{P} ; t\right), L\left(g_{P} ; t\right)$, and $U\left(b_{P} ; t\right)$ are nonempty. Let $x, y \in X$. Then $r_{P}(y) \in[0,1]$. Choose $t=r_{P}(y)$. Thus $r_{P}(y) \leqslant t$, so $y \in L\left(r_{P} ; t\right) \neq \emptyset$. By assumption, we have $L\left(r_{P} ; t\right)$ is a near UP-filter of $X$ and so $x \cdot y \in L\left(r_{P} ; t\right)$. Thus $r_{P}(x \cdot y) \leqslant t=r_{P}(y)$. Let $x, y \in X$. Then $g_{P}(y) \in[0,1]$. Choose $t=g_{P}(y)$. Thus $g_{P}(y) \leqslant t$, so $y \in L\left(g_{P} ; t\right) \neq \emptyset$. By assumption, we have $L\left(g_{P} ; t\right)$ is a near UP-filter of $X$ and so $x \cdot y \in L\left(g_{P} ; t\right)$. Thus $g_{P}(x \cdot y) \leqslant t=g_{P}(y)$. Let $x, y \in X$. Then $b_{P}(y) \in[0,1]$. Choose $t=b_{P}(y)$. Thus $b_{P}(y) \geqslant t$, so $y \in U\left(b_{P} ; t\right) \neq \emptyset$. By assumption, we have $U\left(b_{P} ; t\right)$ is a near UP-filter of $X$ and so $x \cdot y \in U\left(b_{P} ; t\right)$. Thus $b_{P}(x \cdot y) \geqslant t=b_{P}(y)$. Therefore, $P$ is a special picture fuzzy near UP-filter of $X$.

Theorem 4.5. If $\mathrm{P}$ is a special picture fuzzy near $U P$-filter of $\mathrm{X}$, then for all $\mathrm{t} \in[0,1]$, the sets $\mathrm{L}^{-}\left(\mathrm{r}_{\mathrm{P}} ; \mathrm{t}\right), \mathrm{L}^{-}\left(g_{\mathrm{P}} ; \mathrm{t}\right)$, and $\mathrm{U}^{+}\left(\mathrm{b}_{\mathrm{P}} ; \mathrm{t}\right)$ are near $\mathrm{UP}$-filters of $\mathrm{X}$ if $\mathrm{L}^{-}\left(\mathrm{r}_{\mathrm{P}} ; \mathrm{t}\right), \mathrm{L}^{-}\left(\mathrm{g}_{\mathrm{P}} ; \mathrm{t}\right)$, and $\mathrm{U}^{+}\left(\mathrm{b}_{\mathrm{P}} ; \mathrm{t}\right)$ are nonempty.

Proof. Assume that $P$ is a special picture fuzzy near UP-filter of $X$. Let $t \in[0,1]$ be such that $L^{-}\left(r_{P} ; t\right)$, $\mathrm{L}^{-}\left(g_{\mathrm{P}} ; \mathrm{t}\right)$, and $\mathrm{U}^{+}\left(\mathrm{b}_{\mathrm{P}} ; \mathrm{t}\right)$ are nonempty.

Let $x \in X$ and $y \in L^{-}\left(r_{P} ; t\right)$. Then $r_{P}(y)<t$. By (3.4), we have $r_{P}(x \cdot y)<r_{P}(y)<t$. Thus $x \cdot y \in$ $\mathrm{L}^{-}\left(r_{P} ; t\right)$. Let $x \in X$ and $y \in L^{-}\left(g_{P} ; t\right)$. Then $g_{P}(y)<t$. By (3.5), we have $g_{P}(x \cdot y)<g_{P}(y)<t$. Thus 
$x \cdot y \in L^{-}\left(g_{P} ; t\right)$. Let $x \in X$ and $y \in U^{+}\left(b_{P} ; t\right)$. Then $b_{P}(y)>t$. By (3.6), we have $b_{P}(x \cdot y)>b_{P}(y)>t$. Thus $x \cdot y \in \mathrm{U}^{+}\left(b_{P} ; t\right)$. Hence, $\mathrm{L}^{-}\left(\mathrm{r}_{\mathrm{P}} ; t\right), \mathrm{L}^{-}\left(\mathrm{g}_{\mathrm{P}} ; t\right)$, and $\mathrm{U}^{+}\left(\mathrm{b}_{\mathrm{P}} ; t\right)$ are near UP-filters of $X$.

Theorem 4.6. A PFS $\mathrm{P}$ in $\mathrm{X}$ is a special picture fuzzy UP-filter of $\mathrm{X}$ if and only if for all $\mathrm{t} \in[0,1]$, the sets $\mathrm{L}\left(\mathrm{r}_{\mathrm{P}} ; \mathrm{t}\right), \mathrm{L}\left(\mathrm{g}_{\mathrm{P}} ; \mathrm{t}\right)$, and $\mathrm{U}\left(\mathrm{b}_{\mathrm{P}} ; \mathrm{t}\right)$ are $\mathrm{UP}$-filters of $\mathrm{X}$ if $\mathrm{L}\left(\mathrm{r}_{\mathrm{P}} ; \mathrm{t}\right), \mathrm{L}\left(\mathrm{g}_{\mathrm{P}} ; \mathrm{t}\right)$, and $\mathrm{U}\left(\mathrm{b}_{\mathrm{P}} ; \mathrm{t}\right)$ are nonempty.

Proof. Assume that $P$ is a special picture fuzzy UP-filter of $X$. Let $t \in[0,1]$ be such that $L\left(r_{P} ; t\right), L\left(g_{P} ; t\right)$, and $\mathrm{U}\left(\mathrm{b}_{\mathrm{P}} ; \mathrm{t}\right)$ are nonempty.

Let $x \in L\left(r_{P} ; t\right)$. Then $r_{P}(x) \leqslant t$. By (3.7), we have $r_{P}(0) \leqslant r_{P}(x) \leqslant t$. Thus $0 \in L\left(r_{P} ; t\right)$. Next, let $x, y \in X$ be such that $x \cdot y \in L\left(r_{P} ; t\right)$ and $x \in L\left(r_{P} ; t\right)$. Then $r_{P}(x \cdot y) \leqslant t$ and $r_{P}(x) \leqslant t$, so $t$ is an upper bound of $\left\{r_{P}(x \cdot y), r_{P}(x)\right\}$. By (3.10), we have $r_{P}(y) \leqslant \max \left\{r_{P}(x \cdot y), r_{P}(x)\right\} \leqslant t$. Thus $y \in L\left(r_{P} ; t\right)$.

Let $x \in L\left(g_{P} ; t\right)$. Then $g_{P}(x) \leqslant t$. By (3.8), we have $g_{P}(0) \leqslant g_{P}(x) \leqslant t$. Thus $0 \in L\left(g_{P} ; t\right)$. Next, let $x, y \in X$ be such that $x \cdot y \in L\left(g_{P} ; t\right)$ and $x \in L\left(g_{P} ; t\right)$. Then $g_{P}(x \cdot y) \leqslant t$ and $g_{P}(x) \leqslant t$, so $t$ is an upper bound of $\left\{g_{P}(x \cdot y), g_{P}(x)\right\}$. By (3.11), we have $g_{P}(y) \leqslant \max \left\{g_{P}(x \cdot y), g_{P}(x)\right\} \leqslant t$. Thus $y \in L\left(g_{P} ; t\right)$.

Let $x \in U\left(b_{P} ; t\right)$. Then $b_{P}(x) \geqslant t$. By (3.9), we have $b_{P}(0) \geqslant b_{P}(x) \geqslant t$. Thus $0 \in U\left(b_{P} ; t\right)$. Next, let $x, y \in X$ be such that $x \cdot y \in U\left(b_{P} ; t\right)$ and $x \in U\left(b_{P} ; t\right)$. Then $b_{P}(x \cdot y) \geqslant t$ and $b_{P}(x) \geqslant t$, so $t$ is a lower bound of $\left\{b_{P}(x \cdot y), b_{P}(x)\right\}$. By (3.12), we have $b_{P}(y) \geqslant \min \left\{b_{P}(x \cdot y), b_{P}(x)\right\} \geqslant t$. Thus $y \in U\left(b_{P} ; t\right)$.

Hence, $\mathrm{L}\left(\mathrm{r}_{\mathrm{P}} ; \mathrm{t}\right), \mathrm{L}\left(\mathrm{g}_{\mathrm{P}} ; \mathrm{t}\right)$, and $\mathrm{U}\left(\mathrm{b}_{\mathrm{P}} ; \mathrm{t}\right)$ are UP-filters of $X$.

Conversely, assume that for all $t \in[0,1]$, the sets $L\left(r_{P} ; t\right), L\left(g_{P} ; t\right)$, and $U\left(b_{P} ; t\right)$ are UP-filters of $X$ if $\mathrm{L}\left(\mathrm{r}_{\mathrm{P}} ; \mathrm{t}\right), \mathrm{L}\left(\mathrm{g}_{\mathrm{P}} ; \mathrm{t}\right)$, and $\mathrm{U}\left(\mathrm{b}_{\mathrm{P}} ; \mathrm{t}\right)$ are nonempty.

Let $x \in X$. Then $r_{P}(x) \in[0,1]$. Choose $t=r_{P}(x)$. Thus $r_{P}(x) \leqslant t$, so $x \in L\left(r_{P} ; t\right) \neq \emptyset$. By assumption, we have $L\left(r_{P} ; t\right)$ is a UP-filter of $X$ and so $0 \in L\left(r_{P} ; t\right)$. Thus $r_{P}(0) \leqslant t=r_{P}(x)$. Next, let $x, y \in X$. Then $r_{P}(x \cdot y), r_{P}(x) \in[0,1]$. Choose $t=\max \left\{r_{P}(x \cdot y), r_{P}(x)\right\}$. Thus $r_{P}(x \cdot y) \leqslant t$ and $r_{P}(x) \leqslant t$, so $x \cdot y, x \in L\left(r_{P} ; t\right) \neq \emptyset$. By assumption, we have $L\left(r_{P} ; t\right)$ is a UP-filter of $X$ and so $y \in L\left(r_{P} ; t\right)$. Thus $r_{P}(y) \leqslant t=\max \left\{r_{P}(x \cdot y), r_{P}(x)\right\}$.

Let $x \in X$. Then $g_{P}(x) \in[0,1]$. Choose $t=g_{P}(x)$. Thus $g_{P}(x) \leqslant t$, so $x \in L\left(g_{P} ; t\right) \neq \emptyset$. By assumption, we have $L\left(g_{P} ; t\right)$ is a UP-filter of $X$ and so $0 \in L\left(g_{P} ; t\right)$. Thus $g_{P}(0) \leqslant t=g_{P}(x)$. Next, let $x, y \in X$. Then $g_{P}(x \cdot y), g_{P}(x) \in[0,1]$. Choose $t=\max \left\{g_{P}(x \cdot y), g_{P}(x)\right\}$. Thus $g_{P}(x \cdot y) \leqslant t$ and $g_{P}(x) \leqslant t$, so $x \cdot y, x \in L\left(g_{P} ; t\right) \neq \emptyset$. By assumption, we have $L\left(g_{P} ; t\right)$ is a UP-filter of $X$ and so $y \in L\left(g_{P} ; t\right)$. Thus $g_{P}(y) \leqslant t=\max \left\{g_{P}(x \cdot y), g_{P}(x)\right\}$.

Let $x \in X$. Then $b_{P}(x) \in[0,1]$. Choose $t=b_{P}(x)$. Thus $b_{P}(x) \geqslant t$, so $x \in U\left(b_{P} ; t\right) \neq \emptyset$. By assumption, we have $\mathrm{U}\left(\mathrm{b}_{\mathrm{P}} ; t\right)$ is a UP-filter of $X$ and so $0 \in \mathrm{U}\left(\mathrm{b}_{\mathrm{P}} ; t\right)$. Thus $b_{P}(0) \geqslant t=b_{P}(x)$. Next, let $x, y \in X$. Then $b_{P}(x \cdot y), b_{P}(x) \in[0,1]$. Choose $t=\min \left\{b_{P}(x \cdot y), b_{P}(x)\right\}$. Thus $b_{P}(x \cdot y) \geqslant t$ and $b_{P}(x) \geqslant t$, so $x \cdot y, x \in U\left(b_{P} ; t\right) \neq \emptyset$. By assumption, we have $U\left(b_{P} ; t\right)$ is a UP-filter of $X$ and so $y \in U\left(b_{P} ; t\right)$. Thus $b_{P}(y) \geqslant t=\min \left\{b_{P}(x \cdot y), b_{P}(x)\right\}$.

Therefore, $\mathrm{P}$ is a special picture fuzzy UP-filter of $\mathrm{X}$.

Theorem 4.7. If $\mathrm{P}$ is a special picture fuzzy $U P$-filter of $\mathrm{X}$, then for all $\mathrm{t} \in[0,1]$, the sets $\mathrm{L}^{-}\left(\mathrm{r}_{\mathrm{P}} ; \mathrm{t}\right), \mathrm{L}^{-}\left(\mathrm{g}_{\mathrm{P}} ; \mathrm{t}\right)$, and $\mathrm{U}^{+}\left(\mathrm{b}_{\mathrm{P}} ; \mathrm{t}\right)$ are UP-filters of $\mathrm{X}$ if $\mathrm{L}^{-}\left(\mathrm{r}_{\mathrm{P}} ; \mathrm{t}\right), \mathrm{L}^{-}(\mathrm{g} ; \mathrm{t})$, and $\mathrm{U}^{+}\left(\mathrm{b}_{\mathrm{P}} ; \mathrm{t}\right)$ are nonempty.

Proof. Assume that $\mathrm{P}$ is a special picture fuzzy UP-filter of $X$. Let $t \in[0,1]$ be such that $L^{-}\left(r_{P} ; t\right), L^{-}\left(g_{P} ; t\right)$, and $\mathrm{U}^{+}\left(\mathrm{b}_{\mathrm{P}} ; \mathrm{t}\right)$ are nonempty.

Let $x \in \mathrm{L}^{-}\left(\mathrm{r}_{\mathrm{P}} ; t\right)$. Then $\mathrm{r}_{\mathrm{P}}(\mathrm{x})<\mathrm{t}$. By (3.7), we have $\mathrm{r}_{\mathrm{P}}(0)<\mathrm{r}_{\mathrm{P}}(\mathrm{x})<\mathrm{t}$. Thus $0 \in \mathrm{L}^{-}\left(\mathrm{r}_{\mathrm{P}} ; \mathrm{t}\right)$. Next, let $x, y \in X$ be such that $x \cdot y \in \mathrm{L}^{-}\left(r_{P} ; t\right)$ and $x \in \mathrm{L}^{-}\left(r_{P} ; t\right)$. Then $r_{P}(x \cdot y)<t$ and $r_{P}(x)<t$, so $t$ is an upper bound of $\left\{r_{P}(x \cdot y), r_{P}(x)\right\}$. By (3.10), we have $r_{P}(y) \leqslant \max \left\{r_{P}(x \cdot y), r_{P}(x)\right\}<t$. Thus $y \in L^{-}\left(r_{P} ; t\right)$. Let $x \in \mathrm{L}^{-}\left(g_{\mathrm{P}} ; \mathrm{t}\right)$. Then $\mathrm{g}_{\mathrm{P}}(x)<\mathrm{t}$. By (3.8), we have $\mathrm{g}_{\mathrm{P}}(0)<\mathrm{g}_{\mathrm{P}}(\mathrm{x})<\mathrm{t}$. Thus $0 \in \mathrm{L}^{-}\left(\mathrm{g}_{\mathrm{P}} ; \mathrm{t}\right)$. Next, let $x, y \in X$ be such that $x \cdot y \in L^{-}\left(g_{P} ; t\right)$ and $x \in L^{-}\left(g_{P} ; t\right)$. Then $g_{P}(x \cdot y)<t$ and $g_{P}(x)<t$, so $t$ is an upper bound of $\left\{g_{P}(x \cdot y), g_{P}(x)\right\}$. By (3.11), we have $g_{P}(y) \leqslant \max \left\{g_{P}(x \cdot y), g_{P}(x)\right\}<t$. Thus $y \in L^{-}\left(g_{P} ; t\right)$. Let $x \in U^{+}\left(b_{P} ; t\right)$. Then $b_{P}(x)>t$. By (3.9), we have $b_{P}(0)>b_{P}(x)>t$. Thus $0 \in U^{+}\left(b_{P} ; t\right)$. Next, let $x, y \in X$ be such that $x \cdot y \in U^{+}\left(b_{P} ; t\right)$ and $x \in U^{+}\left(b_{P} ; t\right)$. Then $b_{P}(x \cdot y)>t$ and $b_{P}(x)>t$, so $t$ is a lower bound of $\left\{b_{P}(x \cdot y), b_{P}(x)\right\}$. By (3.12), we have $b_{P}(y) \geqslant \min \left\{b_{P}(x \cdot y), b_{P}(x)\right\}>t$. Thus $y \in u^{+}\left(b_{P} ; t\right)$. Hence, $\mathrm{L}^{-}\left(\mathrm{r}_{\mathrm{P}} ; \mathrm{t}\right), \mathrm{L}^{-}\left(g_{P} ; t\right)$, and $\mathrm{U}^{+}\left(b_{P} ; t\right)$ are UP-filters of $X$. 
Theorem 4.8. A PFS $\mathrm{P}$ in $\mathrm{X}$ is a special picture fuzzy implicative UP-filter of $\mathrm{X}$ if and only if for all $\mathrm{t} \in[0,1]$, the sets $\mathrm{L}\left(\mathrm{r}_{\mathrm{P}} ; \mathrm{t}\right), \mathrm{L}\left(\mathrm{g}_{\mathrm{P}} ; \mathrm{t}\right)$, and $\mathrm{U}\left(\mathrm{b}_{\mathrm{P}} ; \mathrm{t}\right)$ are implicative $\mathrm{UP}$-filters of $\mathrm{X}$ if $\mathrm{L}\left(\mathrm{r}_{\mathrm{P}} ; \mathrm{t}\right), \mathrm{L}\left(\mathrm{g}_{\mathrm{P}} ; \mathrm{t}\right)$, and $\mathrm{U}\left(\mathrm{b}_{\mathrm{P}} ; \mathrm{t}\right)$ are nonempty.

Proof. Assume that $P$ is a special picture fuzzy implicative UP-filter of $X$. Let $t \in[0,1]$ be such that $\mathrm{L}\left(\mathrm{r}_{\mathrm{P}} ; \mathrm{t}\right), \mathrm{L}\left(\mathrm{g}_{\mathrm{p}} ; \mathrm{t}\right)$, and $\mathrm{U}\left(\mathrm{b}_{\mathrm{P}} ; \mathrm{t}\right)$ are nonempty.

Let $x \in L\left(r_{P} ; t\right)$. Then $r_{P}(x) \leqslant t$. By (3.7), we have $r_{P}(0) \leqslant r_{P}(x) \leqslant t$. Thus $0 \in L\left(r_{P} ; t\right)$. Next, let $x, y, z \in X$ be such that $x \cdot(y \cdot z) \in L\left(r_{P} ; t\right)$ and $x \cdot y \in L\left(r_{P} ; t\right)$. Then $r_{P}(x \cdot(y \cdot z)) \leqslant t$ and $r_{P}(x \cdot y) \leqslant t$, so $t$ is an upper bound of $\left\{r_{P}(x \cdot(y \cdot z)), r_{P}(x \cdot y)\right\}$. By (3.13), we have $r_{P}(x \cdot z) \leqslant \max \left\{r_{P}(x \cdot(y \cdot z)), r_{P}(x \cdot y)\right\} \leqslant$ t. Thus $x \cdot z \in \mathrm{L}\left(\mathrm{r}_{\mathrm{P}} ; \mathrm{t}\right)$. Let $x \in \mathrm{L}\left(g_{\mathrm{P}} ; \mathrm{t}\right)$. Then $g_{\mathrm{P}}(x) \leqslant \mathrm{t}$. By $(3.8)$, we have $g_{\mathrm{P}}(0) \leqslant g_{\mathrm{P}}(x) \leqslant \mathrm{t}$. Thus $0 \in \mathrm{L}\left(\mathrm{g}_{\mathrm{P}} ; \mathrm{t}\right)$. Next, let $x, y, z \in X$ be such that $x \cdot(y \cdot z) \in \mathrm{L}\left(\mathrm{g}_{\mathrm{P}} ; \mathrm{t}\right)$ and $x \cdot y \in \mathrm{L}\left(\mathrm{g}_{\mathrm{P}} ; \mathrm{t}\right)$. Then $g_{P}(x \cdot(y \cdot z)) \leqslant t$ and $g_{P}(x \cdot y) \leqslant t$, so $t$ is an upper bound of $\left\{g_{P}(x \cdot(y \cdot z)), g_{P}(x \cdot y)\right\}$. By (3.14), we have $g_{P}(x \cdot z) \leqslant \max \left\{g_{P}(x \cdot(y \cdot z)), g_{P}(x \cdot y)\right\} \leqslant t$. Thus $x \cdot z \in L\left(g_{P} ; t\right)$. Let $x \in U\left(b_{P} ; t\right)$. Then $b_{P}(x) \geqslant t$. By (3.9), we have $b_{P}(0) \geqslant b_{P}(x) \geqslant t$. Thus $0 \in U\left(b_{P} ; t\right)$. Next, let $x, y, z \in X$ be such that $x \cdot(y \cdot z) \in U\left(b_{P} ; t\right)$ and $x \cdot y \in U\left(b_{P} ; t\right)$. Then $b_{P}(x \cdot(y \cdot z)) \geqslant t$ and $b_{P}(x \cdot y) \geqslant t$, so $t$ is a lower bound of $\left\{b_{P}(x \cdot(y \cdot z)), b_{P}(x \cdot y)\right\}$. By (3.15), we have $b_{P}(x \cdot z) \geqslant \min \left\{b_{P}(x \cdot(y \cdot z)), b_{P}(x \cdot y)\right\} \geqslant t$. Thus $x \cdot z \in \mathrm{U}\left(\mathrm{b}_{\mathrm{P}} ; \mathrm{t}\right)$. Hence, $\mathrm{L}\left(\mathrm{r}_{\mathrm{P}} ; \mathrm{t}\right), \mathrm{L}\left(\mathrm{g}_{\mathrm{P}} ; \mathrm{t}\right)$, and $\mathrm{U}\left(\mathrm{b}_{\mathrm{P}} ; \mathrm{t}\right)$ are implicative UP-filters of $X$.

Conversely, assume that for all $t \in[0,1]$, the sets $L\left(r_{P} ; t\right), L\left(g_{P} ; t\right)$, and $U\left(b_{P} ; t\right)$ are implicative UPfilters of $X$ if $L\left(r_{P} ; t\right), L\left(g_{P} ; t\right)$, and $U\left(b_{P} ; t\right)$ are nonempty.

Let $x \in X$. Then $r_{P}(x) \in[0,1]$. Choose $t=r_{P}(x)$. Thus $r_{P}(x) \leqslant t$, so $x \in L\left(r_{P} ; t\right) \neq \emptyset$. By assumption, we have $L\left(r_{P} ; t\right)$ is an implicative UP-filter of $X$ and so $0 \in L\left(r_{P} ; t\right)$. Thus $r_{P}(0) \leqslant t=r_{P}(x)$. Next, let $x, y, z \in X$. Then $r_{P}(x \cdot(y \cdot z)), r_{P}(x \cdot y) \in[0,1]$. Choose $t=\max \left\{r_{P}(x \cdot(y \cdot z)), r_{P}(x \cdot y)\right\}$. Thus $r_{P}(x \cdot(y \cdot z)) \leqslant t$ and $r_{P}(x \cdot y) \leqslant t$, so $x \cdot(y \cdot z), x \cdot y \in L\left(r_{P} ; t\right) \neq \emptyset$. By assumption, we have $L\left(r_{P} ; t\right)$ is an implicative UP-filter of $X$ and so $x \cdot z \in L\left(r_{P} ; t\right)$. Thus $r_{P}(x \cdot z) \leqslant t=\max \left\{r_{P}(x \cdot(y \cdot z)), r_{P}(x \cdot y)\right\}$.

Let $x \in X$. Then $g_{P}(x) \in[0,1]$. Choose $t=g_{P}(x)$. Thus $g_{P}(x) \leqslant t$, so $x \in L\left(g_{P} ; t\right) \neq \emptyset$. By assumption, we have $L\left(g_{P} ; t\right)$ is an implicative UP-filter of $X$ and so $0 \in L\left(g_{P} ; t\right)$. Thus $g_{P}(0) \leqslant t=g_{P}(x)$. Next, let $x, y, z \in X$. Then $g_{P}(x \cdot(y \cdot z)), g_{P}(x \cdot y) \in[0,1]$. Choose $t=\max \left\{g_{P}(x \cdot(y \cdot z)), g_{P}(x \cdot y)\right\}$. Thus $g_{P}(x \cdot(y \cdot z)) \leqslant t$ and $g_{P}(x \cdot y) \leqslant t$, so $x \cdot(y \cdot z), x \cdot y \in L\left(g_{P} ; t\right) \neq \emptyset$. By assumption, we have $L\left(g_{P} ; t\right)$ is an implicative UP-filter of $X$ and so $x \cdot z \in L\left(g_{P} ; t\right)$. Thus $g_{P}(x \cdot z) \leqslant t=\max \left\{g_{P}(x \cdot(y \cdot z)), g_{P}(x \cdot y)\right\}$.

Let $x \in X$. Then $b_{P}(x) \in[0,1]$. Choose $t=b_{P}(x)$. Thus $b_{P}(x) \geqslant t$, so $x \in U\left(b_{P} ; t\right) \neq \emptyset$. By assumption, we have $\mathrm{U}\left(\mathrm{b}_{\mathrm{P}} ; t\right)$ is an implicative UP-filter of $X$ and so $0 \in \mathrm{U}\left(\mathrm{b}_{\mathrm{P}} ; t\right)$. Thus $\mathrm{b}_{\mathrm{P}}(0) \geqslant t=\mathrm{b}_{\mathrm{P}}(\mathrm{x})$. Next, let $x, y, z \in X$. Then $b_{P}(x \cdot(y \cdot z)), b_{P}(x \cdot y) \in[0,1]$. Choose $t=\min \left\{b_{P}(x \cdot(y \cdot z)), b_{P}(x \cdot y)\right\}$. Thus $b_{P}(x \cdot(y \cdot z)) \geqslant t$ and $b_{P}(x \cdot y) \geqslant t$, so $x \cdot(y \cdot z), x \cdot y \in U\left(b_{P} ; t\right) \neq \emptyset$. By assumption, we have $U\left(b_{P} ; t\right)$ is an implicative UP-filter of $X$ and so $x \cdot z \in U\left(b_{P} ; t\right)$. Thus $b_{P}(x \cdot z) \geqslant t=\min \left\{b_{P}(x \cdot(y \cdot z)), b_{P}(x \cdot y)\right\}$.

Therefore, $P$ is a special picture fuzzy implicative UP-filter of $X$.

Theorem 4.9. If $\mathrm{P}$ is a special picture fuzzy implicative UP-filter of $\mathrm{X}$, then for all $\mathrm{t} \in[0,1]$, the sets $\mathrm{L}^{-}\left(\mathrm{r}_{\mathrm{P}} ; \mathrm{t}\right)$, $\mathrm{L}^{-}\left(g_{\mathrm{P}} ; \mathrm{t}\right)$, and $\mathrm{U}^{+}\left(\mathrm{b}_{\mathrm{P}} ; \mathrm{t}\right)$ are implicative $\mathrm{UP}$-filters of $\mathrm{X}$ if $\mathrm{L}^{-}\left(\mathrm{r}_{\mathrm{P}} ; \mathrm{t}\right), \mathrm{L}^{-}\left(\mathrm{g}_{\mathrm{P}} ; \mathrm{t}\right)$, and $\mathrm{U}^{+}\left(\mathrm{b}_{\mathrm{P}} ; \mathrm{t}\right)$ are nonempty.

Proof. Assume that $P$ is a special picture fuzzy implicative UP-filter of $X$. Let $t \in[0,1]$ be such that $\mathrm{L}^{-}\left(\mathrm{r}_{\mathrm{P}} ; \mathrm{t}\right), \mathrm{L}^{-}\left(\mathrm{g}_{\mathrm{P}} ; \mathrm{t}\right)$, and $\mathrm{U}^{+}\left(\mathrm{b}_{\mathrm{P}} ; \mathrm{t}\right)$ are nonempty.

Let $x \in \mathrm{L}^{-}\left(\mathrm{r}_{\mathrm{P}} ; t\right)$. Then $\mathrm{r}_{\mathrm{P}}(x)<\mathrm{t}$. By (3.7), we have $\mathrm{r}_{\mathrm{P}}(0)<\mathrm{r}_{\mathrm{P}}(x)<t$. Thus $0 \in \mathrm{L}^{-}\left(\mathrm{r}_{\mathrm{P}} ; \mathrm{t}\right)$. Next, let $x, y, z \in X$ be such that $x \cdot(y \cdot z) \in L^{-}\left(r_{P} ; t\right)$ and $x \cdot y \in L^{-}\left(r_{P} ; t\right)$. Then $r_{P}(x \cdot(y \cdot z))<t$ and $r_{P}(x \cdot y)<t$, so $t$ is an upper bound of $\left\{r_{P}(x \cdot(y \cdot z)), r_{P}(x \cdot y)\right\}$. By (3.13), we have $r_{P}(x \cdot z) \leqslant \max \left\{r_{P}(x \cdot(y \cdot z)), r_{P}(x \cdot y)\right\}<$ t. Thus $\mathrm{x} \cdot z \in \mathrm{L}^{-}\left(\mathrm{r}_{\mathrm{P}} ; \mathrm{t}\right)$.

Let $x \in \mathrm{L}^{-}\left(g_{\mathrm{P}} ; \mathrm{t}\right)$. Then $\mathrm{g}_{\mathrm{P}}(\mathrm{x})<\mathrm{t}$. By (3.8), we have $\mathrm{g}_{\mathrm{P}}(0)<\mathrm{g}_{\mathrm{P}}(\mathrm{x})<\mathrm{t}$. Thus $0 \in \mathrm{L}^{-}\left(\mathrm{g}_{\mathrm{P}} ; \mathrm{t}\right)$. Next, let $x, y, z \in X$ be such that $x \cdot(y \cdot z) \in \mathrm{L}^{-}\left(g_{P} ; t\right)$ and $x \cdot y \in L^{-}\left(g_{P} ; t\right)$. Then $g_{P}(x \cdot(y \cdot z))<t$ and $g_{P}(x \cdot y)<t$, so $t$ is an upper bound of $\left\{g_{P}(x \cdot(y \cdot z)), g_{P}(x \cdot y)\right\}$. By (3.14), we have $g_{P}(x \cdot z) \leqslant$ $\max \left\{g_{\mathrm{P}}(x \cdot(y \cdot z)), g_{\mathrm{P}}(x \cdot y)\right\}<t$. Thus $x \cdot z \in \mathrm{L}^{-}\left(g_{\mathrm{P}} ; \mathrm{t}\right)$.

Let $x \in U^{+}\left(b_{P} ; t\right)$. Then $b_{P}(x)>t$. By (3.9), we have $b_{P}(0)>b_{P}(x)>t$. Thus $0 \in U^{+}\left(b_{P} ; t\right)$. Next, let $x, y, z \in X$ be such that $x \cdot(y \cdot z) \in \mathrm{U}^{+}\left(b_{P} ; t\right)$ and $x \cdot y \in U^{+}\left(b_{P} ; t\right)$. Then $b_{P}(x \cdot(y \cdot z))>t$ and $b_{P}(x \cdot y)>t$, so $t$ is a lower bound of $\left\{b_{P}(x \cdot(y \cdot z)), b_{P}(x \cdot y)\right\}$. By (3.15), we have $b_{P}(x \cdot z) \geqslant$ $\min \left\{b_{P}(x \cdot(y \cdot z)), b_{P}(x \cdot y)\right\}>t$. Thus $x \cdot z \in U^{+}\left(b_{P} ; t\right)$. 
Hence, $\mathrm{L}^{-}\left(\mathrm{r}_{\mathrm{P}} ; \mathrm{t}\right), \mathrm{L}^{-}\left(g_{\mathrm{P}} ; \mathrm{t}\right)$, and $\mathrm{U}^{+}\left(\mathrm{b}_{\mathrm{P}} ; \mathrm{t}\right)$ are implicative UP-filters of $X$.

Theorem 4.10. A PFS $\mathrm{P}$ in $\mathrm{X}$ is a special picture fuzzy comparative UP-filter of $\mathrm{X}$ if and only if for all $\mathrm{t} \in[0,1]$, the sets $\mathrm{L}\left(\mathrm{r}_{\mathrm{P}} ; \mathrm{t}\right), \mathrm{L}\left(\mathrm{g}_{\mathrm{P}} ; \mathrm{t}\right)$, and $\mathrm{U}\left(\mathrm{b}_{\mathrm{P}} ; \mathrm{t}\right)$ are comparative $\mathrm{UP}$-filters of $\mathrm{X}$ if $\mathrm{L}\left(\mathrm{r}_{\mathrm{P}} ; \mathrm{t}\right), \mathrm{L}\left(\mathrm{g}_{\mathrm{P}} ; \mathrm{t}\right)$, and $\mathrm{U}\left(\mathrm{b}_{\mathrm{P}} ; \mathrm{t}\right)$ are nonempty.

Proof. Assume that $P$ is a special picture fuzzy comparative UP-filter of $X$. Let $t \in[0,1]$ be such that $\mathrm{L}\left(\mathrm{r}_{\mathrm{P}} ; \mathrm{t}\right), \mathrm{L}\left(\mathrm{g}_{\mathrm{P}} ; \mathrm{t}\right)$, and $\mathrm{U}\left(\mathrm{b}_{\mathrm{P}} ; \mathrm{t}\right)$ are nonempty.

Let $x \in \mathrm{L}\left(\mathrm{r}_{\mathrm{P}} ; t\right)$. Then $\mathrm{r}_{\mathrm{P}}(x) \leqslant t$. By (3.7), we have $\mathrm{r}_{\mathrm{P}}(0) \leqslant \mathrm{r}_{\mathrm{P}}(x) \leqslant \mathrm{t}$. Thus $0 \in \mathrm{L}\left(\mathrm{r}_{\mathrm{P}} ; \mathrm{t}\right)$. Next, let $x, y, z \in X$ be such that $x \cdot((y \cdot z) \cdot y) \in L\left(r_{P} ; t\right)$ and $x \in L\left(r_{P} ; t\right)$. Then $r_{P}(x \cdot((y \cdot z) \cdot y)) \leqslant t$ and $r_{P}(x) \leqslant t$, so $t$ is an upper bound of $\left\{r_{P}(x \cdot((y \cdot z) \cdot y)), r_{P}(x)\right\}$. By (3.16), we have $r_{P}(y) \leqslant \max \left\{r_{P}(x \cdot((y \cdot z) \cdot y)), r_{P}(x)\right\} \leqslant$ t. Thus $y \in \mathrm{L}\left(\mathrm{r}_{\mathrm{P}} ; \mathrm{t}\right)$.

Let $x \in \mathrm{L}\left(g_{\mathrm{P}} ; \mathrm{t}\right)$. Then $g_{\mathrm{P}}(x) \leqslant \mathrm{t}$. By (3.8), we have $g_{\mathrm{P}}(0) \leqslant g_{\mathrm{P}}(x) \leqslant \mathrm{t}$. Thus $0 \in \mathrm{L}\left(g_{\mathrm{P}} ; \mathrm{t}\right)$. Next, let $x, y, z \in X$ be such that $x \cdot((y \cdot z) \cdot y) \in L\left(g_{P} ; t\right)$ and $x \in L\left(g_{P} ; t\right)$. Then $g_{P}(x \cdot((y \cdot z) \cdot y)) \leqslant t$ and $g_{P}(x) \leqslant t$, so $t$ is an upper bound of $\left\{g_{P}(x \cdot((y \cdot z) \cdot y)), g_{P}(x)\right\}$. By $(3.17)$, we have $g_{P}(y) \leqslant \max \left\{g_{P}(x \cdot((y\right.$. $\left.z) \cdot y)), g_{P}(x)\right\} \leqslant t$. Thus $y \in L\left(g_{P} ; t\right)$.

Let $x \in U\left(b_{P} ; t\right)$. Then $b_{P}(x) \geqslant t$. By (3.9), we have $b_{P}(0) \geqslant b_{P}(x) \geqslant t$. Thus $0 \in U\left(b_{P} ; t\right)$. Next, let $x, y, z \in X$ be such that $x \cdot((y \cdot z) \cdot y) \in U\left(b_{P} ; t\right)$ and $x \in U\left(b_{P} ; t\right)$. Then $b_{P}(x \cdot((y \cdot z) \cdot y)) \geqslant t$ and $b_{P}(x) \geqslant t$, so $t$ is a lower bound of $\left\{b_{P}(x \cdot((y \cdot z) \cdot y)), b_{P}(x)\right\}$. By (3.18), we have $b_{P}(y) \geqslant \min \left\{b_{P}(x \cdot((y\right.$. $\left.z) \cdot y)), b_{P}(x)\right\} \geqslant t$. Thus $y \in U\left(b_{P} ; t\right)$.

Hence, $\mathrm{L}\left(\mathrm{r}_{\mathrm{P}} ; \mathrm{t}\right), \mathrm{L}\left(\mathrm{g}_{\mathrm{P}} ; \mathrm{t}\right)$, and $\mathrm{U}\left(\mathrm{b}_{\mathrm{P}} ; \mathrm{t}\right)$ are comparative UP-filters of $\mathrm{X}$.

Conversely, assume that for all $t \in[0,1]$, the sets $L\left(r_{P} ; t\right), L\left(g_{P} ; t\right)$, and $U\left(b_{P} ; t\right)$ are comparative UPfilters of $X$ if $L\left(r_{P} ; t\right), L\left(g_{P} ; t\right)$, and $U\left(b_{P} ; t\right)$ are nonempty.

Let $x \in X$. Then $r_{P}(x) \in[0,1]$. Choose $t=r_{P}(x)$. Thus $r_{P}(x) \leqslant t$, so $x \in L\left(r_{P} ; t\right) \neq \emptyset$. By assumption, we have $L\left(r_{P} ; t\right)$ is a comparative UP-filter of $X$ and so $0 \in L\left(r_{P} ; t\right)$. Thus $r_{P}(0) \leqslant t=r_{P}(x)$. Next, let $x, y, z \in X$. Then $r_{P}(x \cdot((y \cdot z) \cdot y)), r_{P}(x) \in[0,1]$. Choose $t=\max \left\{r_{P}(x \cdot((y \cdot z) \cdot y)), r_{P}(x)\right\}$. Thus $r_{P}(x \cdot((y \cdot z) \cdot y)) \leqslant t$ and $r_{P}(x) \leqslant t$, so $x \cdot((y \cdot z) \cdot y), x \in L\left(r_{P} ; t\right) \neq \emptyset$. By assumption, we have $L\left(r_{P} ; t\right)$ is a comparative UP-filter of $X$ and so $y \in L\left(r_{P} ; t\right)$. Thus $r_{P}(y) \leqslant t=\max \left\{r_{P}(x \cdot((y \cdot z) \cdot y)), r_{P}(x)\right\}$.

Let $x \in X$. Then $g_{P}(x) \in[0,1]$. Choose $t=g_{P}(x)$. Thus $g_{P}(x) \leqslant t$, so $x \in L\left(g_{P} ; t\right) \neq \emptyset$. By assumption, we have $L\left(g_{P} ; t\right)$ is a comparative UP-filter of $X$ and so $0 \in L\left(g_{P} ; t\right)$. Thus $g_{P}(0) \leqslant t=g_{P}(x)$. Next, let $x, y, z \in X$. Then $g_{P}(x \cdot((y \cdot z) \cdot y)), g_{P}(x) \in[0,1]$. Choose $t=\max \left\{g_{P}(x \cdot((y \cdot z) \cdot y)), g_{P}(x)\right\}$. Thus $g_{P}(x \cdot((y \cdot z) \cdot y)) \leqslant t$ and $g_{P}(x) \leqslant t$, so $x \cdot((y \cdot z) \cdot y), x \in L\left(g_{P} ; t\right) \neq \emptyset$. By assumption, we have $L\left(g_{P} ; t\right)$ is a comparative UP-filter of $X$ and so $y \in L\left(g_{P} ; t\right)$. Thus $g_{P}(y) \leqslant t=\max \left\{g_{P}(x \cdot((y \cdot z) \cdot y)), g_{P}(x)\right\}$.

Let $x \in X$. Then $b_{P}(x) \in[0,1]$. Choose $t=b_{P}(x)$. Thus $b_{P}(x) \geqslant t$, so $x \in U\left(b_{P} ; t\right) \neq \emptyset$. By assumption, we have $\mathrm{U}\left(\mathrm{b}_{\mathrm{P}} ; t\right)$ is a comparative UP-filter of $X$ and so $0 \in \mathrm{U}\left(\mathrm{b}_{\mathrm{P}} ; t\right)$. Thus $b_{P}(0) \geqslant t=b_{P}(x)$. Next, let $x, y, z \in X$. Then $b_{P}(x \cdot((y \cdot z) \cdot y)), b_{P}(x) \in[0,1]$. Choose $t=\min \left\{b_{P}(x \cdot((y \cdot z) \cdot y)), b_{P}(x)\right\}$. Thus $b_{P}(x \cdot((y \cdot z) \cdot y)) \geqslant t$ and $b_{P}(x) \geqslant t$, so $x \cdot((y \cdot z) \cdot y), x \in U\left(b_{P} ; t\right) \neq \emptyset$. By assumption, we have $U\left(b_{P} ; t\right)$ is a comparative UP-filter of $X$ and so $y \in U\left(b_{P} ; t\right)$. Thus $b_{P}(y) \geqslant t=\min \left\{b_{P}(x \cdot((y \cdot z) \cdot y)), b_{P}(x)\right\}$.

Therefore, $P$ is a special picture fuzzy comparative UP-filter of $X$.

Theorem 4.11. If $\mathrm{P}$ is a special picture fuzzy comparative UP-filter of $\mathrm{X}$, then for all $\mathrm{t} \in[0,1]$, the sets $\mathrm{L}^{-}\left(\mathrm{r}_{\mathrm{P}} ; \mathrm{t}\right)$, $\mathrm{L}^{-}\left(\mathrm{g}_{\mathrm{P}} ; \mathrm{t}\right)$, and $\mathrm{U}^{+}\left(\mathrm{b}_{\mathrm{P}} ; \mathrm{t}\right)$ are comparative UP-filters of $\mathrm{X}$ if $\mathrm{L}^{-}\left(\mathrm{r}_{\mathrm{P}} ; \mathrm{t}\right), \mathrm{L}^{-}\left(\mathrm{g}_{\mathrm{P}} ; \mathrm{t}\right)$, and $\mathrm{U}^{+}\left(\mathrm{b}_{\mathrm{P}} ; \mathrm{t}\right)$ are nonempty.

Proof. Assume that $\mathrm{P}$ is a special picture fuzzy comparative UP-filter of $X$. Let $t \in[0,1]$ be such that $\mathrm{L}^{-}\left(\mathrm{r}_{\mathrm{P}} ; \mathrm{t}\right), \mathrm{L}^{-}\left(g_{\mathrm{P}} ; \mathrm{t}\right)$, and $\mathrm{U}^{+}\left(\mathrm{b}_{\mathrm{P}} ; \mathrm{t}\right)$ are nonempty.

Let $x \in \mathrm{L}^{-}\left(\mathrm{r}_{\mathrm{P}} ; \mathrm{t}\right)$. Then $\mathrm{r}_{\mathrm{P}}(x)<\mathrm{t}$. By (3.7), we have $\mathrm{r}_{\mathrm{P}}(0)<\mathrm{r}_{\mathrm{P}}(x)<\mathrm{t}$. Thus $0 \in \mathrm{L}^{-}\left(\mathrm{r}_{\mathrm{P}} ; \mathrm{t}\right)$. Next, let $x, y, z \in X$ be such that $x \cdot((y \cdot z) \cdot y) \in \mathrm{L}^{-}\left(r_{P} ; t\right)$ and $x \in \mathrm{L}^{-}\left(r_{P} ; t\right)$. Then $r_{P}(x \cdot((y \cdot z) \cdot y))<t$ and $r_{P}(x)<t$, so $t$ is an upper bound of $\left\{r_{P}(x \cdot((y \cdot z) \cdot y)), r_{P}(x)\right\}$. By (3.16), we have $r_{P}(y) \leqslant \max \left\{r_{P}(x \cdot((y \cdot\right.$ $\left.z) \cdot y)), r_{P}(x)\right\}<t$. Thus $y \in L^{-}\left(r_{P} ; t\right)$.

Let $x \in \mathrm{L}^{-}\left(g_{\mathrm{P}} ; \mathrm{t}\right)$. Then $\mathrm{g}_{\mathrm{P}}(\mathrm{x})<\mathrm{t}$. By (3.8), we have $\mathrm{g}_{\mathrm{P}}(0)<\mathrm{g}_{\mathrm{P}}(\mathrm{x})<\mathrm{t}$. Thus $0 \in \mathrm{L}^{-}\left(g_{\mathrm{P}} ; \mathrm{t}\right)$. Next, let $x, y, z \in X$ be such that $x \cdot((y \cdot z) \cdot y) \in \mathrm{L}^{-}\left(g_{P} ; t\right)$ and $x \in \mathrm{L}^{-}\left(g_{P} ; t\right)$. Then $g_{P}(x \cdot((y \cdot z) \cdot y))<t$ and $g_{P}(x)<t$, so $t$ is an upper bound of $\left\{g_{P}(x \cdot((y \cdot z) \cdot y)), g_{P}(x)\right\}$. By $(3.17)$, we have $g_{P}(y) \leqslant \max \left\{g_{P}(x \cdot((y\right.$. $\left.z) \cdot y)), g_{P}(x)\right\}<t$. Thus $y \in L^{-}\left(g_{P} ; t\right)$. 
Let $x \in \mathrm{U}^{+}\left(\mathrm{b}_{\mathrm{P}} ; \mathrm{t}\right)$. Then $\mathrm{b}_{\mathrm{P}}(\mathrm{x})>\mathrm{t}$. By (3.9), we have $\mathrm{b}_{\mathrm{P}}(0)>\mathrm{b}_{\mathrm{P}}(\mathrm{x})>\mathrm{t}$. Thus $0 \in \mathrm{U}^{+}\left(\mathrm{b}_{\mathrm{P}} ; \mathrm{t}\right)$. Next, let $x, y, z \in X$ be such that $x \cdot((y \cdot z) \cdot y) \in U^{+}\left(b_{P} ; t\right)$ and $x \in U^{+}\left(b_{P} ; t\right)$. Then $b_{P}(x \cdot((y \cdot z) \cdot y))>t$ and $b_{P}(x)>t$, so $t$ is a lower bound of $\left\{b_{P}(x \cdot((y \cdot z) \cdot y)), b_{P}(x)\right\}$. By (3.18), we have $b_{P}(y) \geqslant \min \left\{b_{P}(x \cdot((y\right.$. $\left.z) \cdot y)), b_{\mathrm{P}}(x)\right\}>t$. Thus $y \in \mathrm{U}^{+}\left(\mathrm{b}_{\mathrm{P}} ; \mathrm{t}\right)$.

Hence, $L^{-}\left(r_{P} ; t\right), L^{-}\left(g_{P} ; t\right)$, and $U^{+}\left(b_{P} ; t\right)$ are comparative UP-filters of $X$.

Theorem 4.12. A PFS $\mathrm{P}$ in $\mathrm{X}$ is a special picture fuzzy shift UP-filter of $\mathrm{X}$ if and only if for all $\mathrm{t} \in[0,1]$, the sets $\mathrm{L}\left(\mathrm{r}_{\mathrm{P}} ; \mathrm{t}\right), \mathrm{L}\left(\mathrm{g}_{\mathrm{P}} ; \mathrm{t}\right)$, and $\mathrm{U}\left(\mathrm{b}_{\mathrm{P}} ; \mathrm{t}\right)$ are shift $\mathrm{UP}$-filters of $\mathrm{X}$ if $\mathrm{L}\left(\mathrm{r}_{\mathrm{P}} ; \mathrm{t}\right), \mathrm{L}\left(\mathrm{g}_{\mathrm{P}} ; \mathrm{t}\right)$, and $\mathrm{U}\left(\mathrm{b}_{\mathrm{P}} ; \mathrm{t}\right)$ are nonempty.

Proof. Assume that $P$ is a special picture fuzzy shift UP-filter of $X$. Let $t \in[0,1]$ be such that $L\left(r_{P} ; t\right)$, $\mathrm{L}\left(g_{\mathrm{P}} ; \mathrm{t}\right)$, and $\mathrm{U}\left(\mathrm{b}_{\mathrm{P}} ; \mathrm{t}\right)$ are nonempty.

Let $x \in L\left(r_{P} ; t\right)$. Then $r_{P}(x) \leqslant t$. By (3.7), we have $r_{P}(0) \leqslant r_{P}(x) \leqslant t$. Thus $0 \in L\left(r_{P} ; t\right)$. Next, let $x, y, z \in X$ be such that $x \cdot(y \cdot z) \in L\left(r_{P} ; t\right)$ and $x \in L\left(r_{P} ; t\right)$. Then $r_{P}(x \cdot(y \cdot z)) \leqslant t$ and $r_{P}(x) \leqslant t$, so $t$ is an upper bound of $\left\{r_{P}(x \cdot(y \cdot z)), r_{P}(x)\right\}$. By (3.19), we have $r_{P}(((z \cdot y) \cdot y) \cdot z) \leqslant \max \left\{r_{P}(x \cdot(y \cdot z)), r_{P}(x)\right\} \leqslant t$. Thus $((z \cdot y) \cdot y) \cdot z \in \mathrm{L}\left(\mathrm{r}_{\mathrm{P}} ; \mathrm{t}\right)$.

Let $x \in \mathrm{L}\left(g_{\mathrm{P}} ; \mathrm{t}\right)$. Then $g_{\mathrm{P}}(x) \leqslant t$. By (3.8), we have $g_{\mathrm{P}}(0) \leqslant g_{\mathrm{P}}(x) \leqslant t$. Thus $0 \in \mathrm{L}\left(g_{\mathrm{P}} ; \mathrm{t}\right)$. Next, let $x, y, z \in X$ be such that $x \cdot(y \cdot z) \in \mathrm{L}\left(g_{P} ; t\right)$ and $x \in L\left(g_{P} ; t\right)$. Then $g_{P}(x \cdot(y \cdot z)) \leqslant t$ and $g_{P}(x) \leqslant t$, so $t$ is an upper bound of $\left\{g_{P}(x \cdot(y \cdot z)), g_{P}(x)\right\}$. By (3.20), we have $g_{P}(((z \cdot y) \cdot y) \cdot z) \leqslant \max \left\{g_{P}(x \cdot(y \cdot z)), g_{P}(x)\right\} \leqslant t$. Thus $((z \cdot y) \cdot y) \cdot z \in \mathrm{L}\left(g_{P} ; \mathrm{t}\right)$.

Let $x \in U\left(b_{P} ; t\right)$. Then $b_{P}(x) \geqslant t$. By (3.9), we have $b_{P}(0) \geqslant b_{P}(x) \geqslant t$. Thus $0 \in U\left(b_{P} ; t\right)$. Next, let $x, y, z \in X$ be such that $x \cdot(y \cdot z) \in U\left(b_{P} ; t\right)$ and $x \in U\left(b_{P} ; t\right)$. Then $b_{P}(x \cdot(y \cdot z)) \geqslant t$ and $b_{P}(x) \geqslant t$, so $t$ is a lower bound of $\left\{b_{P}(x \cdot(y \cdot z)), b_{P}(x)\right\}$. By $(3.21)$, we have $b_{P}(((z \cdot y) \cdot y) \cdot z) \geqslant \min \left\{b_{P}(x \cdot(y \cdot z)), b_{P}(x)\right\} \geqslant t$. Thus $((z \cdot y) \cdot y) \cdot z \in U\left(b_{P} ; t\right)$. Hence, $L\left(r_{P} ; t\right), L\left(g_{P} ; t\right)$, and $U\left(b_{P} ; t\right)$ are shift UP-filters of $X$.

Conversely, assume that for all $t \in[0,1]$, the sets $L\left(r_{P} ; t\right), L\left(g_{P} ; t\right)$, and $U\left(b_{P} ; t\right)$ are shift UP-filters of $X$ if $\mathrm{L}\left(\mathrm{r}_{\mathrm{P}} ; \mathrm{t}\right), \mathrm{L}\left(\mathrm{g}_{\mathrm{P}} ; \mathrm{t}\right)$, and $\mathrm{U}\left(\mathrm{b}_{\mathrm{P}} ; \mathrm{t}\right)$ are nonempty.

Let $x \in X$. Then $r_{P}(x) \in[0,1]$. Choose $t=r_{P}(x)$. Thus $r_{P}(x) \leqslant t$, so $x \in L\left(r_{P} ; t\right) \neq \emptyset$. By assumption, we have $L\left(r_{P} ; t\right)$ is a shift UP-filter of $X$ and so $0 \in L\left(r_{P} ; t\right)$. Thus $r_{P}(0) \leqslant t=r_{P}(x)$. Next, let $x, y, z \in X$. Then $r_{P}(x \cdot(y \cdot z)), r_{P}(x) \in[0,1]$. Choose $t=\max \left\{r_{P}(x \cdot(y \cdot z)), r_{P}(x)\right\}$. Thus $r_{P}(x \cdot(y \cdot z)) \leqslant t$ and $r_{P}(x) \leqslant t$, so $x \cdot(y \cdot z), x \in L\left(r_{P} ; t\right) \neq \emptyset$. By assumption, we have $L\left(r_{P} ; t\right)$ is a shift UP-filter of $X$ and so $((z \cdot y) \cdot y) \cdot z \in \mathrm{L}\left(\mathrm{r}_{\mathrm{P}} ; \mathrm{t}\right)$. Thus $\mathrm{r}_{\mathrm{P}}(((z \cdot y) \cdot \mathrm{y}) \cdot z) \leqslant \mathrm{t}=\max \left\{\mathrm{r}_{\mathrm{P}}(\mathrm{x} \cdot(\mathrm{y} \cdot z)), \mathrm{r}_{\mathrm{P}}(\mathrm{x})\right\}$.

Let $x \in X$. Then $g_{P}(x) \in[0,1]$. Choose $t=g_{P}(x)$. Thus $g_{P}(x) \leqslant t$, so $x \in L\left(g_{P} ; t\right) \neq \emptyset$. By assumption, we have $L\left(g_{P} ; t\right)$ is a shift UP-filter of $X$ and so $0 \in L\left(g_{P} ; t\right)$. Thus $g_{P}(0) \leqslant t=g_{P}(x)$. Next, let $x, y, z \in X$. Then $g_{P}(x \cdot(y \cdot z)), g_{P}(x) \in[0,1]$. Choose $t=\max \left\{g_{P}(x \cdot(y \cdot z)), g_{P}(x)\right\}$. Thus $g_{P}(x \cdot(y \cdot z)) \leqslant t$ and $g_{P}(x) \leqslant t$, so $x \cdot(y \cdot z), x \in L\left(g_{P} ; t\right) \neq \emptyset$. By assumption, we have $L\left(g_{P} ; t\right)$ is a shift UP-filter of $X$ and so $((z \cdot y) \cdot y) \cdot z \in \mathrm{L}\left(g_{P} ; t\right)$. Thus $g_{P}(((z \cdot y) \cdot y) \cdot z) \leqslant t=\max \left\{g_{P}(x \cdot(y \cdot z)), g_{P}(x)\right\}$.

Let $x \in X$. Then $b_{P}(x) \in[0,1]$. Choose $t=b_{P}(x)$. Thus $b_{P}(x) \geqslant t$, so $x \in U\left(b_{P} ; t\right) \neq \emptyset$. By assumption, we have $U\left(b_{P} ; t\right)$ is a shift UP-filter of $X$ and so $0 \in U\left(b_{P} ; t\right)$. Thus $b_{P}(0) \geqslant t=b_{P}(x)$. Next, let $x, y, z \in X$. Then $b_{P}(x \cdot(y \cdot z)), b_{P}(x) \in[0,1]$. Choose $t=\min \left\{b_{P}(x \cdot(y \cdot z)), b_{P}(x)\right\}$. Thus $b_{P}(x \cdot(y \cdot z)) \geqslant t$ and $b_{P}(x) \geqslant t$, so $x \cdot(y \cdot z), x \in U\left(b_{P} ; t\right) \neq \emptyset$. By assumption, we have $U\left(b_{P} ; t\right)$ is a shift UP-filter of $X$ and so $((z \cdot y) \cdot y) \cdot z \in U\left(b_{P} ; t\right)$. Thus $b_{P}(((z \cdot y) \cdot y) \cdot z) \geqslant t=\min \left\{b_{P}(x \cdot(y \cdot z)), b_{P}(x)\right\}$.

Therefore, $P$ is a special picture fuzzy shift UP-filter of $X$.

Theorem 4.13. If $\mathrm{P}$ is a special picture fuzzy shift $U P$-filter of $X$, then for all $t \in[0,1]$, the sets $\mathrm{L}^{-}\left(\mathrm{r}_{\mathrm{P}} ; \mathrm{t}\right), \mathrm{L}^{-}\left(g_{\mathrm{P}} ; \mathrm{t}\right)$, and $\mathrm{U}^{+}\left(\mathrm{b}_{\mathrm{P}} ; \mathrm{t}\right)$ are shift $\mathrm{UP}$-filters of $\mathrm{X}$ if $\mathrm{L}^{-}\left(\mathrm{r}_{\mathrm{P}} ; \mathrm{t}\right), \mathrm{L}^{-}\left(\mathrm{g}_{\mathrm{P}} ; \mathrm{t}\right)$, and $\mathrm{U}^{+}\left(\mathrm{b}_{\mathrm{P}} ; \mathrm{t}\right)$ are nonempty.

Proof. Assume that $P$ is a special picture fuzzy shift UP-filter of $X$. Let $t \in[0,1]$ be such that $L^{-}\left(r_{P} ; t\right)$, $\mathrm{L}^{-}\left(\mathrm{g}_{\mathrm{P}} ; \mathrm{t}\right)$, and $\mathrm{U}^{+}\left(\mathrm{b}_{\mathrm{P}} ; \mathrm{t}\right)$ are nonempty.

Let $x \in \mathrm{L}^{-}\left(\mathrm{r}_{\mathrm{P}} ; \mathrm{t}\right)$. Then $\mathrm{r}_{\mathrm{P}}(\mathrm{x})<\mathrm{t}$. By (3.7), we have $\mathrm{r}_{\mathrm{P}}(0)<\mathrm{r}_{\mathrm{P}}(\mathrm{x})<\mathrm{t}$. Thus $0 \in \mathrm{L}^{-}\left(\mathrm{r}_{\mathrm{P}} ; \mathrm{t}\right)$. Next, let $x, y, z \in X$ be such that $x \cdot(y \cdot z) \in \mathrm{L}^{-}\left(r_{P} ; t\right)$ and $x \in \mathrm{L}^{-}\left(r_{P} ; t\right)$. Then $r_{P}(x \cdot(y \cdot z))<t$ and $r_{P}(x)<t$, so $t$ is an upper bound of $\left\{r_{P}(x \cdot(y \cdot z)), r_{P}(x)\right\}$. By (3.19), we have $r_{P}(((z \cdot y) \cdot y) \cdot z) \leqslant \max \left\{r_{P}(x \cdot(y \cdot z)), r_{P}(x)\right\}<t$. Thus $((z \cdot y) \cdot y) \cdot z \in \mathrm{L}^{-}\left(\mathrm{r}_{\mathrm{P}} ; \mathrm{t}\right)$. 
Let $x \in \mathrm{L}^{-}\left(g_{\mathrm{P}} ; \mathrm{t}\right)$. Then $\mathrm{g}_{\mathrm{P}}(\mathrm{x})<\mathrm{t}$. By (3.8), we have $\mathrm{g}_{\mathrm{P}}(0)<\mathrm{g}_{\mathrm{P}}(\mathrm{x})<\mathrm{t}$. Thus $0 \in \mathrm{L}^{-}\left(g_{\mathrm{P}} ; \mathrm{t}\right)$. Next, let $x, y, z \in X$ be such that $x \cdot(y \cdot z) \in \mathrm{L}^{-}\left(g_{P} ; t\right)$ and $x \in \mathrm{L}^{-}\left(g_{P} ; t\right)$. Then $g_{P}(x \cdot(y \cdot z))<t$ and $g_{P}(x)<t$, so $t$ is an upper bound of $\left\{g_{\mathrm{P}}(x \cdot(y \cdot z)), g_{\mathrm{P}}(x)\right\}$. By (3.20), we have $g_{\mathrm{P}}(((z \cdot y) \cdot y) \cdot z) \leqslant \max \left\{g_{\mathrm{P}}(x \cdot(y \cdot z)), g_{\mathrm{P}}(x)\right\}<$ t. Thus $((z \cdot y) \cdot y) \cdot z \in \mathrm{L}^{-}\left(g_{P} ; t\right)$.

Let $x \in \mathrm{U}^{+}\left(b_{\mathrm{P}} ; t\right)$. Then $b_{\mathrm{P}}(x)>t$. By (3.9), we have $b_{P}(0)>b_{P}(x)>t$. Thus $0 \in U^{+}\left(b_{P} ; t\right)$. Next, let $x, y, z \in X$ be such that $x \cdot(y \cdot z) \in U^{+}\left(b_{P} ; t\right)$ and $x \in U^{+}\left(b_{P} ; t\right)$. Then $b_{P}(x \cdot(y \cdot z))>t$ and $b_{P}(x)>t$, so $t$ is a lower bound of $\left\{b_{P}(x \cdot(y \cdot z)), b_{P}(x)\right\}$. By (3.21), we have $\left.b_{P}((z \cdot y) \cdot y) \cdot z\right) \geqslant \min \left\{b_{P}(x \cdot(y \cdot z)), b_{P}(x)\right\}>t$. Thus $((z \cdot y) \cdot y) \cdot z \in U^{+}\left(b_{P} ; t\right)$.

Hence, $\mathrm{L}^{-}\left(\mathrm{r}_{\mathrm{P}} ; \mathrm{t}\right), \mathrm{L}^{-}\left(g_{P} ; t\right)$, and $\mathrm{U}^{+}\left(b_{\mathrm{P}} ; t\right)$ are shift UP-filters of $X$.

Theorem 4.14. A PFS $\mathrm{P}$ in $\mathrm{X}$ is a special picture fuzzy UP-ideal of $\mathrm{X}$ if and only if for all $\mathrm{t} \in[0,1]$, the sets $\mathrm{L}\left(\mathrm{r}_{\mathrm{P}} ; \mathrm{t}\right), \mathrm{L}\left(\mathrm{g}_{\mathrm{P}} ; \mathrm{t}\right)$, and $\mathrm{U}\left(\mathrm{b}_{\mathrm{P}} ; \mathrm{t}\right)$ are $\mathrm{UP}$-ideals of $\mathrm{X}$ if $\mathrm{L}\left(\mathrm{r}_{\mathrm{P}} ; \mathrm{t}\right), \mathrm{L}\left(\mathrm{g}_{\mathrm{P}} ; \mathrm{t}\right)$, and $\mathrm{U}\left(\mathrm{b}_{\mathrm{P}} ; \mathrm{t}\right)$ are nonempty.

Proof. Assume that $P$ is a special picture fuzzy UP-ideal of $X$. Let $t \in[0,1]$ be such that $L\left(r_{P} ; t\right), L\left(g_{P} ; t\right)$, and $\mathrm{U}\left(\mathrm{b}_{\mathrm{P}} ; \mathrm{t}\right)$ are nonempty.

Let $x \in \mathrm{L}\left(\mathrm{r}_{\mathrm{P}} ; t\right)$. Then $\mathrm{r}_{\mathrm{P}}(x) \leqslant t$. By (3.7), we have $\mathrm{r}_{\mathrm{P}}(0) \leqslant \mathrm{r}_{\mathrm{P}}(x) \leqslant \mathrm{t}$. Thus $0 \in \mathrm{L}\left(\mathrm{r}_{\mathrm{P}} ; \mathrm{t}\right)$. Next, let $x, y, z \in X$ be such that $x \cdot(y \cdot z) \in L\left(r_{P} ; t\right)$ and $y \in L\left(r_{P} ; t\right)$. Then $r_{P}(x \cdot(y \cdot z)) \leqslant t$ and $r_{P}(y) \leqslant t$, so $t$ is an upper bound of $\left\{r_{P}(x \cdot(y \cdot z)), r_{P}(y)\right\}$. By (3.22), we have $r_{P}(x \cdot z) \leqslant \max \left\{r_{P}(x \cdot(y \cdot z)), r_{P}(y)\right\} \leqslant t$. Thus $x \cdot z \in \mathrm{L}\left(\mathrm{r}_{\mathrm{P}} ; \mathrm{t}\right)$.

Let $x \in \mathrm{L}\left(g_{\mathrm{P}} ; \mathrm{t}\right)$. Then $g_{\mathrm{P}}(x) \leqslant t$. By (3.8), we have $g_{\mathrm{P}}(0) \leqslant g_{\mathrm{P}}(x) \leqslant t$. Thus $0 \in \mathrm{L}\left(g_{\mathrm{P}} ; \mathrm{t}\right)$. Next, let $x, y, z \in X$ be such that $x \cdot(y \cdot z) \in L\left(g_{P} ; t\right)$ and $y \in L\left(g_{P} ; t\right)$. Then $g_{P}(x \cdot(y \cdot z)) \leqslant t$ and $g_{P}(y) \leqslant t$, so $t$ is an upper bound of $\left\{g_{P}(x \cdot(y \cdot z)), g_{P}(y)\right\}$. By (3.23), we have $g_{P}(x \cdot z) \leqslant \max \left\{g_{P}(x \cdot(y \cdot z)), g_{P}(y)\right\} \leqslant t$. Thus $x \cdot z \in \mathrm{L}\left(g_{\mathrm{p}} ; \mathrm{t}\right)$.

Let $x \in U\left(b_{P} ; t\right)$. Then $b_{P}(x) \geqslant t$. By (3.9), we have $b_{P}(0) \geqslant b_{P}(x) \geqslant t$. Thus $0 \in U\left(b_{P} ; t\right)$. Next, let $x, y, z \in X$ be such that $x \cdot(y \cdot z) \in U\left(b_{P} ; t\right)$ and $y \in U\left(b_{P} ; t\right)$. Then $b_{P}(x \cdot(y \cdot z)) \geqslant t$ and $b_{P}(y) \geqslant t$, so $t$ is a lower bound of $\left\{b_{P}(x \cdot(y \cdot z)), b_{P}(y)\right\}$. By (3.24), we have $b_{P}(x \cdot z) \geqslant \min \left\{b_{P}(x \cdot(y \cdot z)), b_{P}(y)\right\} \geqslant t$. Thus $x \cdot z \in \mathrm{U}\left(\mathrm{b}_{\mathrm{P}} ; \mathrm{t}\right)$. Hence, $\mathrm{L}\left(\mathrm{r}_{\mathrm{P}} ; \mathrm{t}\right), \mathrm{L}\left(\mathrm{g}_{\mathrm{P}} ; \mathrm{t}\right)$, and $\mathrm{U}\left(\mathrm{b}_{\mathrm{P}} ; \mathrm{t}\right)$ are UP-ideals of $X$.

Conversely, assume that for all $t \in[0,1]$, the sets $L\left(r_{P} ; t\right), L\left(g_{P} ; t\right)$, and $U\left(b_{P} ; t\right)$ are UP-ideals of $X$ if $\mathrm{L}\left(\mathrm{r}_{\mathrm{P}} ; \mathrm{t}\right), \mathrm{L}\left(\mathrm{g}_{\mathrm{P}} ; \mathrm{t}\right)$, and $\mathrm{U}\left(\mathrm{b}_{\mathrm{P}} ; \mathrm{t}\right)$ are nonempty.

Let $x \in X$. Then $r_{P}(x) \in[0,1]$. Choose $t=r_{P}(x)$. Thus $r_{P}(x) \leqslant t$, so $x \in L\left(r_{P} ; t\right) \neq \emptyset$. By assumption, we have $L\left(r_{P} ; t\right)$ is a UP-ideal of $X$ and so $0 \in L\left(r_{P} ; t\right)$. Thus $r_{P}(0) \leqslant t=r_{P}(x)$. Next, let $x, y, z \in X$. Then $r_{P}(x \cdot(y \cdot z)), r_{P}(y) \in[0,1]$. Choose $t=\max \left\{r_{P}(x \cdot(y \cdot z)), r_{P}(y)\right\}$. Thus $r_{P}(x \cdot(y \cdot z)) \leqslant t$ and $r_{P}(y) \leqslant t$, so $x \cdot(y \cdot z), y \in L\left(r_{P} ; t\right) \neq \emptyset$. By assumption, we have $L\left(r_{P} ; t\right)$ is a UP-ideal of $X$ and so $x \cdot z \in L\left(r_{P} ; t\right)$. Thus $\mathrm{r}_{\mathrm{P}}(\mathrm{x} \cdot z) \leqslant \mathrm{t}=\max \left\{\mathrm{r}_{\mathrm{P}}(\mathrm{x} \cdot(\mathrm{y} \cdot z)), \mathrm{r}_{\mathrm{P}}(\mathrm{y})\right\}$.

Let $x \in X$. Then $g_{P}(x) \in[0,1]$. Choose $t=g_{P}(x)$. Thus $g_{P}(x) \leqslant t$, so $x \in L\left(g_{P} ; t\right) \neq \emptyset$. By assumption, we have $L\left(g_{P} ; t\right)$ is a UP-ideal of $X$ and so $0 \in L\left(g_{P} ; t\right)$. Thus $g_{P}(0) \leqslant t=g_{P}(x)$. Next, let $x, y, z \in X$. Then $g_{P}(x \cdot(y \cdot z)), g_{P}(y) \in[0,1]$. Choose $t=\max \left\{g_{P}(x \cdot(y \cdot z)), g_{P}(y)\right\}$. Thus $g_{P}(x \cdot(y \cdot z)) \leqslant t$ and $g_{P}(y) \leqslant t$, so $x \cdot(y \cdot z), y \in L\left(g_{P} ; t\right) \neq \emptyset$. By assumption, we have $L\left(g_{P} ; t\right)$ is a UP-ideal of $X$ and so $x \cdot z \in L\left(g_{P} ; t\right)$. Thus $g_{\mathrm{P}}(x \cdot z) \leqslant t=\max \left\{g_{\mathrm{P}}(x \cdot(y \cdot z)), g_{\mathrm{P}}(y)\right\}$.

Let $x \in X$. Then $b_{P}(x) \in[0,1]$. Choose $t=b_{P}(x)$. Thus $b_{P}(x) \geqslant t$, so $x \in U\left(b_{P} ; t\right) \neq \emptyset$. By assumption, we have $U\left(b_{P} ; t\right)$ is a UP-ideal of $X$ and so $0 \in U\left(b_{P} ; t\right)$. Thus $b_{P}(0) \geqslant t=b_{P}(x)$. Next, let $x, y, z \in X$. Then $b_{P}(x \cdot(y \cdot z)), b_{P}(y) \in[0,1]$. Choose $t=\min \left\{b_{P}(x \cdot(y \cdot z)), b_{P}(y)\right\}$. Thus $b_{P}(x \cdot(y \cdot z)) \geqslant t$ and $b_{P}(y) \geqslant t$, so $x \cdot(y \cdot z), y \in U\left(b_{P} ; t\right) \neq \emptyset$. By assumption, we have $U\left(b_{P} ; t\right)$ is a UP-ideal of $X$ and so $x \cdot z \in U\left(b_{P} ; t\right)$. Thus $b_{P}(x \cdot z) \geqslant t=\min \left\{b_{P}(x \cdot(y \cdot z)), b_{P}(y)\right\}$.

Therefore, $\mathrm{P}$ is a special picture fuzzy UP-ideal of $\mathrm{X}$.

Theorem 4.15. If $\mathrm{P}$ is a special picture fuzzy $U P$-ideal of $\mathrm{X}$, then for all $\mathrm{t} \in[0,1]$, the sets $\mathrm{L}^{-}\left(\mathrm{r}_{\mathrm{P}} ; \mathrm{t}\right), \mathrm{L}^{-}\left(g_{\mathrm{P}} ; \mathrm{t}\right)$, and $\mathrm{U}^{+}\left(\mathrm{b}_{\mathrm{P}} ; \mathrm{t}\right)$ are $\mathrm{UP}$-ideals of $\mathrm{X}$ if $\mathrm{L}^{-}\left(\mathrm{r}_{\mathrm{P}} ; \mathrm{t}\right), \mathrm{L}^{-}\left(\mathrm{g}_{\mathrm{P}} ; \mathrm{t}\right)$, and $\mathrm{U}^{+}\left(\mathrm{b}_{\mathrm{P}} ; \mathrm{t}\right)$ are nonempty.

Proof. Assume that $\mathrm{P}$ is a special picture fuzzy UP-ideal of $X$. Let $t \in[0,1]$ be such that $\mathrm{L}^{-}\left(\mathrm{r}_{\mathrm{P}} ; \mathrm{t}\right), \mathrm{L}^{-}\left(\mathrm{g}_{\mathrm{P}} ; \mathrm{t}\right)$, and $\mathrm{U}^{+}\left(\mathrm{b}_{\mathrm{P}} ; \mathrm{t}\right)$ are nonempty. 
Let $x \in \mathrm{L}^{-}\left(\mathrm{r}_{\mathrm{P}} ; \mathrm{t}\right)$. Then $\mathrm{r}_{\mathrm{P}}(\mathrm{x})<\mathrm{t}$. By (3.7), we have $\mathrm{r}_{\mathrm{P}}(0)<\mathrm{r}_{\mathrm{P}}(\mathrm{x})<\mathrm{t}$. Thus $0 \in \mathrm{L}^{-}\left(\mathrm{r}_{\mathrm{P}} ; \mathrm{t}\right)$. Next, let $x, y, z \in X$ be such that $x \cdot(y \cdot z) \in L^{-}\left(r_{P} ; t\right)$ and $y \in L^{-}\left(r_{P} ; t\right)$. Then $r_{P}(x \cdot(y \cdot z))<t$ and $r_{P}(y)<t$, so $t$ is an upper bound of $\left\{r_{P}(x \cdot(y \cdot z)), r_{P}(y)\right\}$. By (3.22), we have $r_{P}(x \cdot z) \leqslant \max \left\{r_{P}(x \cdot(y \cdot z)), r_{P}(y)\right\}<t$. Thus $x \cdot z \in \mathrm{L}^{-}\left(\mathrm{r}_{\mathrm{P}} ; \mathrm{t}\right)$. Let $x \in \mathrm{L}^{-}\left(g_{\mathrm{P}} ; \mathrm{t}\right)$. Then $\mathrm{g}_{\mathrm{P}}(x)<\mathrm{t}$. By $(3.8)$, we have $g_{\mathrm{P}}(0)<g_{\mathrm{P}}(x)<t$. Thus $0 \in \mathrm{L}^{-}\left(g_{\mathrm{P}} ; \mathrm{t}\right)$. Next, let $x, y, z \in X$ be such that $x \cdot(y \cdot z) \in \mathrm{L}^{-}\left(g_{\mathrm{P}} ; \mathrm{t}\right)$ and $y \in \mathrm{L}^{-}\left(g_{\mathrm{P}} ; \mathrm{t}\right)$. Then $g_{P}(x \cdot(y \cdot z))<t$ and $g_{P}(y)<t$, so $t$ is an upper bound of $\left\{g_{P}(x \cdot(y \cdot z)), g_{P}(y)\right\}$. By (3.23), we have $g_{P}(x \cdot z) \leqslant \max \left\{g_{P}(x \cdot(y \cdot z)), g_{P}(y)\right\}<t$. Thus $x \cdot z \in L^{-}\left(g_{P} ; t\right)$. Let $x \in U^{+}\left(b_{P} ; t\right)$. Then $b_{P}(x)>t$. By (3.9), we have $b_{P}(0)>b_{P}(x)>t$. Thus $0 \in U^{+}\left(b_{P} ; t\right)$. Next, let $x, y, z \in X$ be such that $x \cdot(y \cdot z) \in U^{+}\left(b_{P} ; t\right)$ and $y \in U^{+}\left(b_{P} ; t\right)$. Then $b_{P}(x \cdot(y \cdot z))>t$ and $b_{P}(y)>t$, so $t$ is a lower bound of $\left\{b_{P}(x \cdot(y \cdot z)), b_{P}(y)\right\}$. By (3.24), we have $b_{P}(x \cdot z) \geqslant \min \left\{b_{P}(x \cdot(y \cdot z)), b_{P}(y)\right\}>t$. Thus $x \cdot z \in U^{+}\left(b_{P} ; t\right)$. Hence, $L^{-}\left(r_{P} ; t\right), L^{-}\left(g_{P} ; t\right)$, and $\mathrm{U}^{+}\left(b_{P} ; t\right)$ are UP-ideals of $X$.

Theorem 4.16. A PFS $\mathrm{P}$ in $\mathrm{X}$ is a special picture fuzzy strong UP-ideal of $\mathrm{X}$ if and only if the sets $\mathrm{E}\left(\mathrm{r}_{\mathrm{P}} ; \mathrm{r}_{\mathrm{P}}(0)\right)$, $\mathrm{E}\left(\mathrm{g}_{\mathrm{P}} ; \mathrm{g}_{\mathrm{P}}(0)\right)$, and $\mathrm{E}\left(\mathrm{b}_{\mathrm{P}} ; \mathrm{b}_{\mathrm{P}}(0)\right)$ are strong UP-ideals of $\mathrm{X}$.

Proof. Assume that $\mathrm{P}$ is a special picture fuzzy strong UP-ideal of $\mathrm{X}$. By Theorem 3.19, we have $\mathrm{P}$ is constant, that is, $r_{P}, g_{P}$, and $b_{P}$ are constant. Thus

$$
(\forall x \in X)\left(\begin{array}{l}
r_{\mathrm{P}}(x)=r_{\mathrm{P}}(0) \\
g_{\mathrm{P}}(x)=g_{\mathrm{P}}(0) \\
b_{\mathrm{P}}(x)=b_{\mathrm{P}}(0)
\end{array}\right) .
$$

Hence, $E\left(r_{P} ; r_{P}(0)\right)=X, E\left(g_{P} ; g_{P}(0)\right)=X$, and $E\left(b_{P} ; b_{P}(0)\right)=X$ and so $E\left(r_{P} ; r_{P}(0)\right), E\left(g_{P} ; g_{P}(0)\right)$, and $E\left(b_{P} ; b_{P}(0)\right)$ are strong UP-ideals of $X$.

Conversely, assume that $E\left(r_{P} ; r_{P}(0)\right), E\left(g_{P} ; g_{P}(0)\right)$, and $E\left(b_{P} ; b_{P}(0)\right)$ are strong UP-ideals of $X$. Then $\mathrm{E}\left(\mathrm{r}_{\mathrm{P}} ; \mathrm{r}_{\mathrm{P}}(0)\right)=\mathrm{X}, \mathrm{E}\left(\mathrm{g}_{\mathrm{P}} ; \mathrm{g}_{\mathrm{P}}(0)\right)=\mathrm{X}$, and $\mathrm{E}\left(\mathrm{b}_{\mathrm{P}} ; \mathrm{b}_{\mathrm{P}}(0)\right)=\mathrm{X}$ and so

$$
(\forall x \in X)\left(\begin{array}{l}
r_{P}(x)=r_{P}(0) \\
g_{P}(x)=g_{P}(0) \\
b_{P}(x)=b_{P}(0)
\end{array}\right) .
$$

Thus $r_{P}, g_{P}$, and $b_{P}$ are constant, that is, $P$ is constant. By Theorem 3.19, we have $P$ is a special picture fuzzy strong UP-ideal of $X$.

Definition 4.17. Let $P$ be a PFS in X. For any $\alpha, \beta, \gamma \in[0,1]$, the sets

$$
\begin{aligned}
\operatorname{UUL}_{\mathrm{P}}(\alpha, \beta, \gamma) & =\left\{x \in X \mid \mathrm{r}_{\mathrm{P}}(x) \geqslant \alpha, g_{P}(x) \geqslant \beta, b_{P}(x) \leqslant \gamma\right\}, \\
\operatorname{LLU}_{\mathrm{P}}(\alpha, \beta, \gamma) & =\left\{x \in X \mid \mathrm{r}_{\mathrm{P}}(x) \leqslant \alpha, g_{P}(x) \leqslant \beta, b_{P}(x) \geqslant \gamma\right\}, \\
E_{P}(\alpha, \beta, \gamma) & =\left\{x \in X \mid r_{P}(x)=\alpha, g_{P}(x)=\beta, b_{P}(x)=\gamma\right\}
\end{aligned}
$$

are called a UUL- $(\alpha, \beta, \gamma)$-level subset, a LLU- $(\alpha, \beta, \gamma)$-level subset, and an E- $(\alpha, \beta, \gamma)$-level subset of $P$, respectively. Then we see that

$$
\begin{aligned}
\operatorname{UUL}_{P}(\alpha, \beta, \gamma) & =\mathrm{U}\left(\mathrm{r}_{P} ; \alpha\right) \cap \mathrm{U}\left(\mathrm{g}_{P} ; \beta\right) \cap \mathrm{L}\left(\mathrm{b}_{P} ; \gamma\right), \\
\operatorname{LLU}_{\mathrm{P}}(\alpha, \beta, \gamma) & =\mathrm{L}\left(\mathrm{r}_{P} ; \alpha\right) \cap \mathrm{L}\left(\mathrm{g}_{P} ; \beta\right) \cap \mathrm{U}\left(\mathrm{b}_{P} ; \gamma\right), \\
\mathrm{E}_{P}(\alpha, \beta, \gamma) & =\mathrm{E}\left(\mathrm{r}_{P} ; \alpha\right) \cap \mathrm{E}\left(g_{P} ; \beta\right) \cap \mathrm{E}\left(\mathrm{b}_{P} ; \gamma\right) .
\end{aligned}
$$

Corollary 4.18. A PFS $P$ in $X$ is a special picture fuzzy UP-subalgebra of $X$ if and only if for all $\alpha, \beta, \gamma \in$ $[0,1], \operatorname{LLU}_{\mathrm{P}}(\alpha, \beta, \gamma)$ is a UP-subalgebra of $\mathrm{X}$ if $\operatorname{LLU}_{\mathrm{P}}(\alpha, \beta, \gamma)$ is nonempty.

Proof. It is straightforward by Theorems 4.2 and 2.5 .

Corollary 4.19. A PFS $P$ in $X$ is a special picture fuzzy near UP-filter of $X$ if and only if for all $\alpha, \beta, \gamma \in$ $[0,1], \operatorname{LLU}_{P}(\alpha, \beta, \gamma)$ is a near UP-filter of $X$ if $\operatorname{LLU}_{P}(\alpha, \beta, \gamma)$ is nonempty.

Proof. It is straightforward by Theorems 4.4 and 2.5 . 
Corollary 4.20. A PFS $P$ in $X$ is a special picture fuzzy UP-filter of $X$ if and only if for all $\alpha, \beta, \gamma \in[0,1]$, $\operatorname{LLU}_{\mathrm{P}}(\alpha, \beta, \gamma)$ is a UP-filter of $\mathrm{X}$ if $\operatorname{LLU}_{\mathrm{P}}(\alpha, \beta, \gamma)$ is nonempty.

Proof. It is straightforward by Theorems 4.6 and 2.5 .

Corollary 4.21. A PFS $\mathrm{P}$ in $\mathrm{X}$ is a special picture fuzzy implicative UP-filter of $\mathrm{X}$ if and only if for all $\alpha, \beta, \gamma \in$ $[0,1], \operatorname{LLU}_{P}(\alpha, \beta, \gamma)$ is a implicative UP-filter of $X$ if $\operatorname{LLU}_{\mathrm{P}}(\alpha, \beta, \gamma)$ is nonempty.

Proof. It is straightforward by Theorems 4.8 and 2.5 .

Corollary 4.22. A PFS $\mathrm{P}$ in $\mathrm{X}$ is a special picture fuzzy comparative UP-filter of $\mathrm{X}$ if and only if for all $\alpha, \beta, \gamma \in$ $[0,1], \operatorname{LLU}_{P}(\alpha, \beta, \gamma)$ is a comparative UP-filter of $X$ if $\operatorname{LLU}_{P}(\alpha, \beta, \gamma)$ is nonempty.

Proof. It is straightforward by Theorems 4.10 and 2.5 .

Corollary 4.23. A PFS $P$ in $X$ is a special picture fuzzy shift UP-filter of $X$ if and only if for all $\alpha, \beta, \gamma \in$ $[0,1], \operatorname{LLU}_{P}(\alpha, \beta, \gamma)$ is a shift UP-filter of $X$ if $\operatorname{LLU}_{P}(\alpha, \beta, \gamma)$ is nonempty.

Proof. It is straightforward by Theorems 4.12 and 2.5 .

Corollary 4.24. A PFS $\mathrm{P}$ in $\mathrm{X}$ is a special picture fuzzy UP-ideal of $\mathrm{X}$ if and only if for all $\alpha, \beta, \gamma \in[0,1]$, $\operatorname{LLU}_{P}(\alpha, \beta, \gamma)$ is a UP-ideal of $X$ if $\operatorname{LLU}_{P}(\alpha, \beta, \gamma)$ is nonempty.

Proof. It is straightforward by Theorems 4.14 and 2.5 .

Corollary 4.25. A PFS $\mathrm{P}$ in $\mathrm{X}$ is a special picture fuzzy strong $U P$-ideal of $\mathrm{X}$ if and only if $\mathrm{E}_{\mathrm{P}}\left(\mathrm{r}_{\mathrm{P}}(0), g_{\mathrm{P}}(0), \mathrm{b}_{\mathrm{P}}(0)\right)$ is a strong UP-ideal of $\mathrm{X}$, that is, $\mathrm{E}\left(\mathrm{r}_{\mathrm{P}}, \mathrm{r}_{\mathrm{P}}(0)\right)=\mathrm{X}, \mathrm{E}\left(\mathrm{g}_{\mathrm{P}}, \mathrm{g}_{\mathrm{P}}(0)\right)=\mathrm{X}$, and $\mathrm{E}\left(\mathrm{b}_{\mathrm{P}}, \mathrm{b}_{\mathrm{P}}(0)\right)=\mathrm{X}$.

Proof. It is straightforward by Theorems 4.16 and 2.5 .

\section{Conclusions}

In this paper, we have introduced the eight new concepts of picture fuzzy sets by means of a special type: special picture fuzzy UP-subalgebras, special picture fuzzy near UP-filters, special picture fuzzy UP-filters, special picture fuzzy implicative UP-filters, special picture fuzzy comparative UP-filters, special picture fuzzy shift UP-filters, special picture fuzzy UP-ideals, and special picture fuzzy strong UP-ideals of UP-algebras and investigated some of their important properties. Then, we get the diagram of generalization of PFSs in UP-algebras as shown in Figure 1 and sufficient conditions of PFSs as shown in Figure 2.

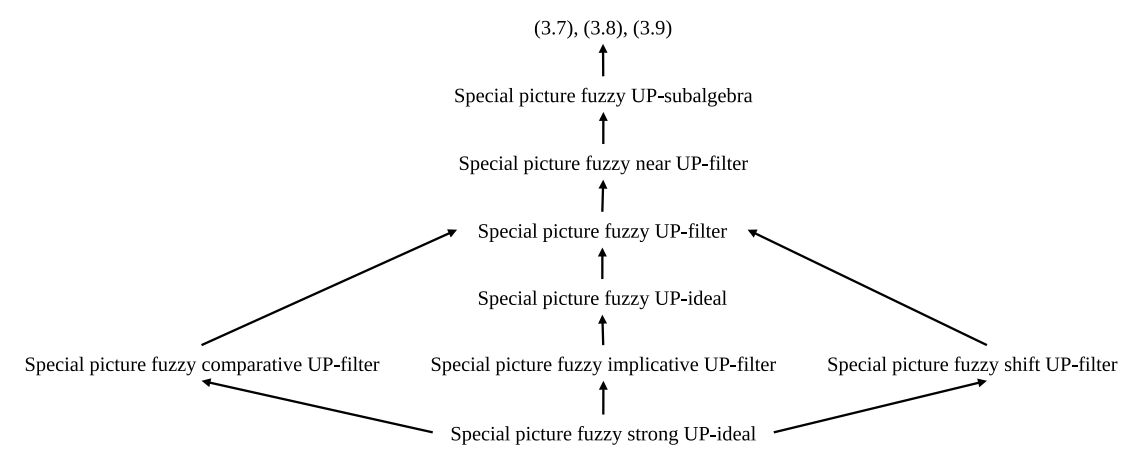

Figure 1: PFSs in UP-algebras by means of a special type 


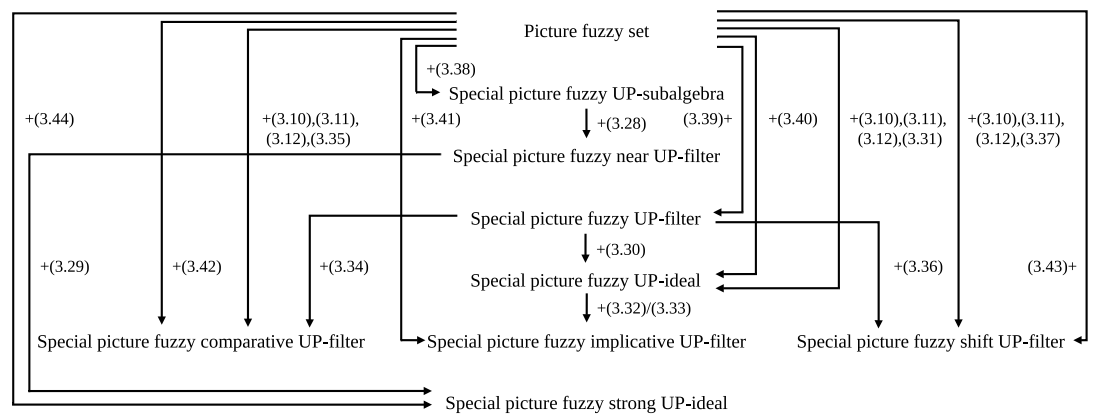

Figure 2: Sufficient conditions of PFSs in UP-algebras

In our future study, we will apply the concepts/results to other type of PFSs in UP-algebras. Also, we will study interval-valued picture fuzzy sets/soft sets/cubic sets/rough sets of PFSs in UP-algebras.

\section{Acknowledgment}

This work was supported by the Unit of Excellence in Mathematics, University of Phayao.

\section{References}

[1] M. A. Ansari, A. Haidar, A. N. A. Koam, On a graph associated to UP-algebras, Math. Comput. Appl., 23 (2018), 12 pages. 2

[2] M. A. Ansari, A. N. A. Koam, A. Haider, Rough set theory applied to UP-algebras, Ital. J. Pure Appl. Math., 42 (2019), 388-402. 2

[3] K. T. Atanassov, Intuitionistic fuzzy sets, Fuzzy Sets and Systems, 20 (1986), 87-96. 1

[4] K. T. Atanassov, New operations defined over the intuitionistic fuzzy sets, Fuzzy Sets and Systems, 61 (1994), $137-142$. 1

[5] B. C. Cuong, Picture fuzzy sets, J. Comput. Sci. Cybernet., 30 (2014), 409-420. 1

[6] B. C. Cuong, V. Kreinovich, Picture fuzzy sets-a new concept for computational intelligence problems, Proceedings of the Third World Congress on Information and Communication Technologies WIICT, (2013), 1-6. 1, 3

[7] N. Dokkhamdang, A. Kesorn, A. Iampan, Generalized fuzzy sets in UP-algebras, Ann. Fuzzy Math. Inform., 16 (2018), 171-190. 2.3

[8] A. H. Ganie, S. Singh, P. K. Bhatia, Some new correlation coefficients of picture fuzzy sets with applications, Neural Comput. Appl., 32 (2020), 12609-12625. 1

[9] T. Guntasow, S. Sajak, A. Jomkham, A. Iampan, Fuzzy translations of a fuzzy set in UP-algebras, J. Indones. Math. Soc., 23 (2017), 1-19. 2.4, 2

[10] A. Iampan, A new branch of the logical algebra: UP-algebras, J. Algebra Relat. Topics, 5 (2017), 35-54. 1, 2.1 , 2, 2, 2.4

[11] A. Iampan, Introducing fully UP-semigroups, Discuss. Math. Gen. Algebra Appl., 38 (2018), 297-306. 1, 2, 2

[12] A. Iampan, Multipliers and near UP-filters of UP-algebras, J. Discrete Math. Sci. Cryptogr., (2019), 1-14. 2.4

[13] A. Iampan, A. Satirad, M. Songsaeng, A note on UP-hyperalgebras, J. Algebr. Hyperstruct. Log. Algebr., 1 (2020), 77-95. 1

[14] A. Iampan, M. Songsaeng, G. Muhiuddin, Fuzzy duplex UP-algebras, Eur. J. Pure Appl. Math., 13 (2020), $459-471$. 2

[15] Y. Imai, K. Iséki, On axiom systems of propositional calculi. XIV, Proc. Japan Acad., 42 (1966), 19-22. 1

[16] K. Iséki, An algebra related with a propositional calculus, Proc. Japan Acad., 42 (1966), 26-29. 1

[17] H. S. Kim, Y. H. Kim, On BE-algebras, Sci. Math. Jpn., 66 (2007), 113-116. 1

[18] C. Prabpayak, U. Leerawat, On ideals and congruences in KU-algebras, Sci. Magna, 5 (2009), 54-57. 2

[19] A. Satirad, R. Chinram, A. Iampan, Four new concepts of extensions of KU/UP-algebras, Missouri J. Math. Sci., 32 (2020), 138-157. 1

[20] A. Satirad, A. Iampan, Topological UP-algebras, Discuss. Math. Gen. Algebra Appl., 39 (2019), 231-250. 1

[21] A. Satirad, P. Mosrijai, A. Iampan, Formulas for finding UP-algebras, Int. J. Math. Comput. Sci., 14 (2019), $403-409$. 2

[22] A. Satirad, P. Mosrijai, A. Iampan, Generalized power UP-algebras, Int. J. Math. Comput. Sci., 14 (2019), 17-25. 2.2

[23] T. Senapati, Y. B. Jun, K. P. Shum, Cubic set structure applied in UP-algebras, Discrete Math. Algorithms Appl., 10 (2018), 23 pages. 
[24] T. Senapati, G. Muhiuddin, K. P. Shum, Representation of UP-algebras in interval-valued intuitionistic fuzzy environment, Ital. J. Pure Appl. Math., 38 (2017), 497-517. 2

[25] P. Singh, Correlation coefficients for picture fuzzy sets, J. Intell. Fuzzy Systems, 28 (2015), 591-604. 1

[26] J. Somjanta, N. Thuekaew, P. Kumpeangkeaw, A. Iampan, Fuzzy sets in UP-algebras, Ann. Fuzzy Math. Inform., 12 (2016), 739-756. 2.4, 4.1

[27] G. Wei, Some cosine similarity measures for picture fuzzy sets and their applications to strategic decision making, Informatica, 28 (2017), 547-564. 1

[28] G. Wei, Some similarity measures for picture fuzzy sets and their applcations, Iran. J. Fuzzy Syst., 15 (2018), 77-89. 1

[29] G. Wei, H. Gao, The generalized Dice similarity measures for picture fuzzy sets and their applications, Informatica, 29 (2018), 107-124. 1

[30] L. A. Zadeh, Fuzzy sets, Inform. Control, 8 (1965), 338-353. 1 